Teoria de Homotopia Simples e Torção de Whitehead

\author{
Luciana Vale Silva
}


SERVIÇO DE PÓS-GRADUAÇÃO DO ICMC-USP

Data de Depósito : 17 / 04 / 2007

Assinatura :

\title{
Teoria de Homotopia Simples e Torção de Whitehead
}

\author{
Luciana Vale Silva \\ Orientador: Prof. Dr. Mauro Flavio Spreafico \\ Dissertação apresentada ao Instituto de Ciências \\ Matemáticas e de Computação - ICMC-USP, \\ como parte dos requisitos para obtenção do título \\ de Mestre em Matemática.
}

USP - São Carlos

Abril de 2007 



\section{Agradecimentos}

Meus primeiros agradecimentos são dedicados ao meu orientador, Prof. Dr. Mauro Flavio Spreafico, por sua excelente orientação, e ao Prof. Dr. Oziride Manzoli Neto, por sua solicitude quando sua ajuda se mostrou indispensável.

Merecem também agradecimentos meus colegas da USP, em particular meu companheiro de estudos Luiz Roberto Hartmann Junior, os funcionários da USP que em geral mostraram-se sempre dispostos a ajudar, meus amigos, enfim todas as pessoas que de alguma forma contribuíram para o desenvolvimento deste projeto.

Não poderia deixar de agradecer à CAPES, pelo apoio financeiro que me possibilitou participar do Programa de Mestrado.

Agradeço em especial à minha família, Fátima, Francisco e Luiz, e ao meu

querido Marcos, pelo apoio fundamental que me deram. É a estas pessoas muito amadas por mim que dedico este trabalho. 



\section{Resumo}

Este trabalho apresenta a teoria de homotopia simples, desenvolvida por J. H. C. Whitehead, com o objetivo de obter um método para classificar espaços com o mesmo tipo de homotopia. Com esta motivação, Whitehead introduz o conceito de equivalência de homotopia simples entre complexos simpliciais, que posteriormente é generalizado para complexos CW, espaços criados pelo próprio Whitehead. Um resultado imediato desta teoria é que quando dois espaços têm o mesmo tipo de homotopia simples, eles têm o mesmo tipo de homotopia. A recíproca desta afirmação é então conjecturada. Mostraremos que trata-se de uma conjectura falsa, contudo a investigação de sua confirmação produz um material que toma rumo próprio. Nosso enfoque são os aspectos algébricos envolvidos nesta investigação. 



\section{Abstract}

This work presents the simple-homotopy theory, developed by J. H. C. Whitehead, with the goal to get an method to classify spaces with the same homotopy type. So, with this motivation, Whitehead introduced the concept of simple-homotopy equivalence between simplicial complexes, that later was generalized for CW complexes, spaces created by himself. An immediate result of this theory is that, if two spaces have the same simple-homotopy type, they have the same homotopy type. Then, the reciprocal statement is conjectured. We will show that the conjecture is not true, but the research about its truthfulness produces a material that takes its own way. Our approach are the algebraic aspects involved in this research. 



\section{Sumário}

$\begin{array}{lll}1 & \text { Introdução } & 1\end{array}$

1.1 Complexos CW . . . . . . . . . . . . . . . . . . 1

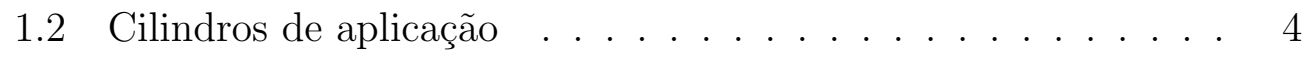

1.3 Complexo de cadeia celular . . . . . . . . . . . . . 5

1.4 Espaços de recobrimento para complexos CW . . . . . . . . . 6

\begin{tabular}{llr}
\hline 2 & Homotopia Simples & 9
\end{tabular}

2.1 Deformações formais . . . . . . . . . . . . . . . . . . . . . 9

2.2 Cilindros de aplicação e deformações $\ldots$. . . . . . . . . . . . . 12

2.3 O grupo de Whitehead de um complexo CW . . . . . . . . . . 14

2.4 Simplificação de pares CW homotopicamente triviais . . . . . 18

2.5 Matrizes e deformações formais . . . . . . . . . . . . . . . 22

$\begin{array}{lll}3 & \text { Álgebra } & 27\end{array}$

3.1 Convenções $\ldots \ldots \ldots$. . . . . . . . . . . . . . . . . . . 27

$3.2 \quad$ Os grupos $K_{G}(R) \ldots \ldots \ldots \ldots \ldots \ldots$

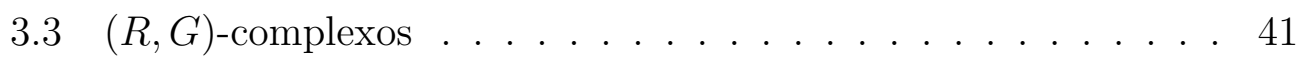

3.4 Complexos de cadeia acíclicos . . . . . . . . . . . . 43

3.5 Equivalência estável de complexos de cadeia acíclicos . . . . . 50 
3.6 Torção de um $(R, G)$-complexo acíclico . . . . . . . . . . . . . 54

3.7 Caracterização da torção de um $(R, G)$-complexo acíclico . . . 58

3.8 Mudança de anéis . . . . . . . . . . . . . . . . . . . . . . . . . 63

4 Torção de Whitehead de um par CW 66

4.1 Definição de torção de Whitehead de um par CW . . . . . . . 66

4.2 Propriedades fundamentais da torção de um par . . . . . . . . 73

$4.3 \quad$ A equivalência natural entre $W h(L)$ e $\oplus W h\left(\pi_{1} L_{j}\right)$. . . . . . . 74

4.4 A torção de uma equivalência de homotopia $\ldots . . . .76$

4.5 Relação entre homotopia e homotopia simples . . . . . . . . . 79

Referências Bibliográficas 



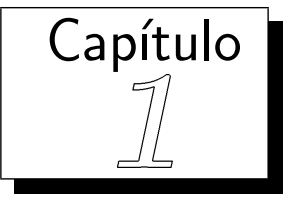

\section{Introdução}

Neste capítulo faremos uma breve exposição sobre os conceitos e resultados básicos que serão utilizados no decorrer deste trabalho.

Começaremos com os complexos CW, espaços sobre os quais é desenvolvida a teoria de homotopia simples.

\subsection{Complexos CW}

Definição 1.1. Um complexo $C W$ é um espaço Hausdorff junto com uma família $\left\{e_{\alpha}\right\}$ de células abertas de dimensões variadas de forma que as seguintes condições são satisfeitas:

(i) $K=\bigcup_{\alpha} e_{\alpha}$ e $e_{\alpha} \cap e_{\beta}=\emptyset$ sempre que $\alpha \neq \beta$.

(ii) Para cada célula $e_{\alpha}$, existe uma aplicação contínua $\varphi_{\alpha}: Q^{n} \rightarrow K$, onde $Q^{n}$ é uma bola topológica de dimensão $n=\operatorname{dim} e_{\alpha}$, ou seja, $Q^{n}$ é homeomorfa a $I^{n}=[0,1]^{n}$, tal que:

(a) $\varphi_{\alpha} \mid \stackrel{\circ}{Q^{n}}: \stackrel{\circ}{Q^{n}} \rightarrow e_{\alpha}$ é um homeomorfismo.

(b) $\varphi_{\alpha}\left(\partial Q^{n}\right) \subset K^{n-1}$, onde $K^{j}=\bigcup\left\{e_{\beta} \mid \operatorname{dim} e_{\beta} \leq j\right\}$. 
(iii) Cada $\bar{e}_{\beta}$ está contido numa união finita de células $e_{\alpha}$.

(iv) Um conjunto $F \subset K$ é fechado em $K$ se e somente se $F \cap \bar{e}_{\alpha}$ é fechado em $\bar{e}_{\alpha}$ para todo $\alpha$.

Uma aplicação $\varphi_{\alpha}$ como em (ii) é chamada de aplicação característica para a célula $e_{\alpha}$.

A aplicação $\varphi_{\alpha} \mid \partial Q^{n}$ é chamada de aplicação colagem para $e_{\alpha}$.

Convenção 1. Neste trabalho vamos lidar apenas com aplicações que são contínuas. Deste modo, se $f: X \rightarrow Y$ é uma aplicação contínua, vamos escrever apenas que $f$ é uma aplicação, ficando subentendido sua continuidade.

Em geral, vamos utilizar como domínio para as aplicações características o espaço $I^{n}$.

Denotaremos o conjunto $\overline{\left(\partial I^{n}-I^{n-1} \times 0\right)}$ por $J^{n-1}$. Observe que este conjunto é topologicamente uma bola de dimensão $n-1$. Também denotaremos o conjunto $\left(I^{n-1} \times 0\right)$ simplesmente por $I^{n-1}$.

Definição 1.2. Um subcomplexo de um complexo CW $K$ é um subconjunto $L$ junto com uma subfamília $\left\{e_{\beta}\right\}$ de células de $K$ tal que $L=\bigcup e_{\beta}$ e cada $\bar{e}_{\beta}$ está contido em $L$. Neste caso, escrevemos $L<K$ e chamamos $(K, L)$ de par $C W$.

Facilmente podem ser verificados que, nas condições acima, $L$ é um subconjunto fechado de $K$, o subespaço $L$ e a família $\left\{e_{\beta}\right\}$ constituem um complexo CW e se e é uma célula de $K$ que não está em $\left\{e_{\beta}\right\}$, então $e \cap L=\emptyset$ (escrevemos $e \in K-L$ ).

Definição 1.3. Dizemos que dois complexos CW $K$ e $K^{\prime}$ são isomorfos e escrevemos $K \cong K^{\prime}$, se existe um homeomorfismo $h: K \rightarrow K^{\prime}$ tal que a imagem de cada célula de $K$ é uma célula de $K^{\prime}$. Chamamos $h$ de isomorfismo $C W$

Teorema 1.1 (Teorema de extensão por homotopia). Seja $(K, L)$ um par $C W$. Dadas uma aplicação $f: K \rightarrow X$, onde $X$ é um espaço qualquer, $e$ 
uma homotopia $f_{t}: L \rightarrow X$ tal que $f_{0}=f \mid L$, então existe uma homotopia $F_{t}$ tal que $F_{0}=f$ e $F_{t} \mid L=f_{t}$. (Referência: [11])

É conveniente neste momento recordar a seguinte definição:

Definição 1.4. Sejam $X$ um espaço e $Y \subset X$. Dizemos que $D: X \rightarrow Y$ é uma retração por deformação forte se existe uma aplicação $F: X \times I \rightarrow X$ tal que:

(a) $F(x, 0)=x, \forall x \in X$,

(b) $F(y, t)=y, \forall y \in Y, t \in I$,

(c) $F(x, 1)=D(x), \forall x \in X$.

Neste caso, denotamos $X{ }^{2} Y$.

Teorema 1.2. Se $(K, L)$ é um par $C W$, então as seguintes afirmações são equivalentes:

(i) $K \longrightarrow L$.

(ii) A aplicação inclusão $i: L \rightarrow K$ é uma equivalência de homotopia.

(iii) $\pi_{n}(K, L)=0, \forall n$.

(Referência [5])

Devido a este teorema, quando um par CW $(K, L)$ satisfaz $K{ }_{\downarrow} L$, dizemos que este par é homotopicamente trivial.

Definição 1.5. Sejam $K$ e $K^{\prime}$ dois complexos CW, dizemos que uma aplicação $f: K \rightarrow K^{\prime}$ é celular se $f\left(K^{n}\right) \subset\left(K^{\prime}\right)^{n}$. Mais geralmente, se $(K, L)$ e $\left(K^{\prime}, L^{\prime}\right)$ são pares CW, dizemos que uma aplicação $f:(K, L) \rightarrow\left(K^{\prime}, L^{\prime}\right)$ é celular quando $f\left(K^{n} \cup L\right) \subset\left(K^{\prime}\right)^{n} \cup L$.

Definição 1.6. Se $f$ é homotópica a uma aplicação celular $g$, então dizemos que $g$ é uma aproximação celular para $f$. 
Teorema 1.3 (Teorema da aproximação celular). Qualquer aplicação entre pares $C W, f:(K, L) \rightarrow\left(K^{\prime}, L^{\prime}\right)$, é homotópica (rel $\left.L\right)$ a uma aplicação celular. (Referência: [11])

\subsection{Cilindros de aplicação}

Definição 1.7. Seja $f: X \rightarrow Y$ uma aplicação. Então o cilindro da aplicação $f$ é o espaço $M_{f}$ obtido tomando a união disjunta de $X \times I$ e $Y$ (escrevemos $(X \times I) \oplus Y)$ e identificando cada $(x, 1)$ com $f(x)$, ou seja,

$$
M_{f}=\frac{(X \times I) \oplus Y}{(x, 1)=f(x)} .
$$

A aplicação identificação $(X \times I) \oplus Y \rightarrow M_{f}$ será sempre denotada por $q$. Observe que $q \mid(X \times[0,1))$ e $q \mid Y$ são imersões, por isso vamos identificar $q(X \times 0) \operatorname{com} X$ e $q(Y)$ com $Y$. Também vamos escrever $q(z)=[z]$.

Proposição 1.4. Seja $f: X \rightarrow Y$ uma aplicação. Então $p: M_{f} \rightarrow Y$ definida por

$$
\begin{aligned}
p[x, t] & =[x, 1]=[f(x)], \quad x \in X, t<1 \\
p[y] & =[y], \quad y \in Y
\end{aligned}
$$

é uma retração por deformação forte. (Referência: [7])

Proposição 1.5. Sejam $f: X \rightarrow Y$ uma aplicação e $i: X \rightarrow M_{f}$ a aplicação inclusão, então:

(i) O seguinte diagrama é comutativo:

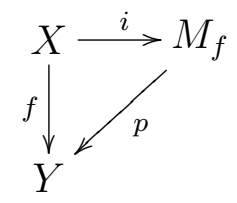

(ii) i é uma equivalência de homotopia se e somente se f é uma equivalência de homotopia. 
Sejam $A$ um subconjunto fechado de um espaço $X$ e $f: A \rightarrow Y$ uma aplicação, então $X \cup \cup_{f} Y$ é o espaço $[X \oplus Y \mid x=f(x), \forall x \in A]$.

Proposição 1.6. Sejam $K, K_{0}$ e $L$ complexos $C W$ com $K_{0}<K$ e seja $f: K_{0} \rightarrow L$ uma aplicação tal que, dada qualquer célula e de $K-K_{0}$, $f\left(\bar{e} \cap K_{0}\right) \subset L^{n-1}$, onde $n=\operatorname{dim} e$. Então $K \cup_{f} L$ é um complexo $C W$ cujas células são as de $K-K_{0}$ e as de $L$. (Mais precisamente, as células de $K \cup_{f} L$ são da forma $q(e)$, onde e é uma célula arbitrária de $K-K_{0}$ ou de $L$ e $q: K \oplus L \rightarrow K \cup_{f} L$ é a aplicação identificação.)

Usando este resultado e a estrutura celular natural de $K \times I$, obtemos:

Proposição 1.7. Se $f: K \rightarrow L$ é uma aplicação celular então o cilindro de aplicação $M_{f}$ é um complexo $C W$ cujas células são as de $L$, as e $\times 0$ e as $e \times(0,1)$, onde e é uma célula qualquer de $K$.

Proposição 1.8. Uma aplicação celular $f: K \rightarrow L$ é uma equivalência de homotopia se e somente se $M_{f}{ }^{2} K$.

\subsection{Complexo de cadeia celular}

Definição 1.8. Seja $(K, L)$ um par CW. O complexo de cadeia celular deste par, $C(K, L)$, é definido da seguinte forma: $C_{n}(K, L)=H_{n}\left(K^{n} \cup L, K^{n-1} \cup L\right)$ e $d_{n}: C_{n}(K, L) \rightarrow C_{n-1}(K, L)$ é o operador bordo da seqüência exata do trio $\left(K^{n} \cup L, K^{n-1} \cup L, K^{n-2} \cup L\right)$.

Para cada $n$, fixemos uma orientação para cada $I^{n}, n=0,1,2, \ldots$, escolhendo um gerador $\omega_{0}$ para $H_{0}\left(I^{0}\right)$ e estipulando que $\omega_{n}$ é obtido a partir de $\omega_{n-1}$, da seguinte forma: a seqüência

$$
H_{n}\left(I^{n}, \partial I^{n}\right) \stackrel{\partial}{\rightarrow} H_{n-1}\left(\partial I^{n}\right) \stackrel{\subset}{\rightarrow} H_{n-1}\left(\partial I^{n}, J^{n-1}\right) \stackrel{(\text { excisão })^{-1}}{\longrightarrow} H_{n-1}\left(I^{n-1}, \partial I^{n-1}\right)
$$

forma um isomorfismo de $H_{n}\left(I^{n}, \partial I^{n}\right)$ em $H_{n-1}\left(I^{n-1}, \partial I^{n-1}\right)$; então definimos $-\omega_{n}$ como sendo a imagem inversa de $\omega_{n-1}$. 
Seja $\varphi_{\alpha}: I^{n} \rightarrow K$ uma aplicação característica para a célula $e_{\alpha}$ de $K-L$. Considere a aplicação induzida $\left(\varphi_{\alpha}\right)_{*}: H_{n}\left(I^{n}, \partial I^{n}\right) \rightarrow H_{n}\left(K^{n} \cup L, K^{n-1} \cup L\right)$. Denote $\left\langle\varphi_{\alpha}\right\rangle=\left(\varphi_{\alpha}\right)_{*}\left(\omega_{n}\right)$.

Lema 1.9. Escolha uma aplicação característica $\varphi_{\alpha}$ para cada n-célula $e_{\alpha}$ de $K-$ L. Denote $K_{j}=K^{j} \cup L$. Então:

(i) $H_{j}\left(K_{n}, K_{n-1}\right)=0$ se $j \neq n$.

(ii) $H_{n}\left(K_{n}, K_{n-1}\right)$ é livre com base $\left\{\left\langle\varphi_{\alpha}\right\rangle \mid e_{\alpha}^{n} \in K-L\right\}$.

(iii) Se c é um n-ciclo singular de $K \bmod L$ representando $\gamma \in$ $H_{n}\left(K_{n}, K_{n-1}\right)$ e se $|c|$ não inclui a n-célula $e_{\alpha_{0}}$, então $n_{\alpha_{0}}=0$ na expressão $\gamma=\sum_{\alpha} n_{\alpha}\left\langle\varphi_{\alpha}\right\rangle$.

(Referências: [16] e [11])

\subsection{Espaços de recobrimento para complexos CW}

Nesta seção vamos convencionar que os espaços base dos recobrimentos são sempre conexos.

Teorema 1.10. Seja $K$ um complexo $C W$. Então para todo grupo $G<\pi_{1} K$ existe um recobrimento $p: E \rightarrow K$ tal que $p_{\sharp}\left(\pi_{1} E\right)=G$. Em particular, $K$ tem espaço de recobrimento universal. (Referência: [11])

Definição 1.9. Dizemos que um recobrimento $p: E \rightarrow K$ é um recobrimento na categoria $C W$ se $E$ e $K$ são complexos $C W$ e se a imagem de cada célula de $E$ é uma célula de $K$.

Proposição 1.11. Sejam $K$ um complexo $C W$ e $p: E \rightarrow K$ um recobrimento de K. Então

$$
\left\{\tilde{e}_{\alpha} \mid e_{\alpha} \in K, \tilde{e}_{\alpha} \text { é um levantamento de } e_{\alpha} \text { para } E\right\}
$$

é uma estrutura celular para $E$ com respeito da qual E torna-se um complexo $C W$. Se $\varphi_{\alpha}: I^{n} \rightarrow K$ é uma aplicação característica para a célula $e_{\alpha}, \tilde{e}_{\alpha}$ 
é um levantamento de $e_{\alpha}$ e $\tilde{\varphi}_{\alpha}: I^{n} \rightarrow E$ é um levantamento de $\varphi_{\alpha}$ tal que $\tilde{\varphi}_{\alpha}(x) \in \tilde{e}_{\alpha}$, para algum $x \in I^{n}$, então $\tilde{\varphi}_{\alpha}$ é uma aplicação característica para $\tilde{e}_{\alpha} \cdot($ Referência: [11])

Devido à proposição acima, convencionaremos que um recobrimento que tem como espaço base um complexo CW será sempre um recobrimento na categoria CW.

Proposição 1.12. Sejam $K$ e $K^{\prime}$ complexos $C W, p: E \rightarrow K$ recobrimento e $f: K^{\prime} \rightarrow K$ uma aplicação celular que tem um levantamento $\tilde{f}: K^{\prime} \rightarrow E$. Então $\tilde{f}$ é celular e se $f$ é um recobrimento (na categoria $C W$ ), $\tilde{f}$ também é.

Corolário 1.13. Se $K$ um complexo $C W$, então o espaço de recobrimento universal de $K$ é único a menos de isomorfismo celular.

Proposição 1.14. Sejam $(K, L)$ um par de complexos $C W$ conexos e $p$ : $\tilde{K} \rightarrow K$ um recobrimento universal. Defina $\tilde{L}=p^{-1}(L)$. Se a aplicação $i_{\sharp}: \pi_{1} L \rightarrow \pi_{1} K$, induzida pela inclusão $i: L \rightarrow K$, é um isomorfismo, então $p \mid \tilde{L}: \tilde{L} \rightarrow L$ é um recobrimento universal de $L$. Se, além disso, $K{ }_{\downarrow} L$, então $\tilde{K} \aleph_{\downarrow} \tilde{L}$. (Referência: [5])

Proposição 1.15. Seja $f: K \rightarrow L$ uma aplicação celular entre complexos $C W$ conexos tal que $f_{\sharp}: \pi_{1} K \rightarrow \pi_{1} L$ é um isomorfismo. Se $\tilde{K}$ e $\tilde{L}$ são espaços de recobrimento universal de $K$ e L, respectivamente, e se $\tilde{f}: \tilde{K} \rightarrow \tilde{L}$ é um levantamento de $f$, então $M_{\tilde{f}}$ é um espaço de recobrimento universal de $M_{f}$. (Referência: [5])

Agora, sejam $(K, L)$ um par CW finito, $p: \tilde{K} \rightarrow K$ um recobrimento universal e $\tilde{L}=p^{-1}(L)$. Considere o complexo de cadeia celular $C(\tilde{K}, \tilde{L})$. Temos, pelo resultado 1.9 , que cada $C_{n}(\tilde{K}, \tilde{L})$ é um $\mathbb{Z}$-módulo livre.

Defina $G=\operatorname{Cov}(\tilde{K})=\{h: \tilde{K} \rightarrow \tilde{K} \mid h$ é homeomorfismo e $p h=p\}$.

Por 1.12, cada $g \in G$ é um isomorfismo celular de $\tilde{K}$ que induz um homomorfismo $g_{*}: C_{n}(\tilde{K}, \tilde{L}) \rightarrow C_{n}(\tilde{K}, \tilde{L})$, para cada $n$, e satisfaz $d g_{*}=g_{*} d$ (onde $d$ é o operador bordo de $C(\tilde{K}, \tilde{L})$ ). 
Considere a ação de $G$ em $C_{n}(\tilde{K}, \tilde{L})$ dada por

$$
g \cdot c=g_{*}(c), \quad g \in G, c \in C_{n}(\tilde{K}, \tilde{L}) .
$$

Claramente $d(g \cdot c)=g \cdot d(c)$. Assim, $C_{n}(\tilde{K}, \tilde{L})$ torna-se um $\mathbb{Z} G$-módulo se definirmos

$$
\left(\sum_{i} n_{i} g_{i}\right) \cdot c=\sum_{i} n_{i}\left(g_{i} \cdot c\right)=\sum_{i} n_{i}\left(g_{i}\right)_{*}(c) .
$$

Teorema 1.16. Sejam $(K, L)$ um par $C W, p: \tilde{K} \rightarrow K$ um recobrimento universal, $\tilde{L}=p^{-1}(L)$ e $G=\operatorname{Cov}(\tilde{K})$. Para cada n-célula $e_{\alpha}$ de $K-L$ fixe um aplicação característica $\varphi_{\alpha}: I^{n} \rightarrow K$ e um levantamento $\tilde{\varphi}_{\alpha}: I^{n} \rightarrow \tilde{K}$ específico de $\varphi_{\alpha}$. Então $\left\{\left\langle\tilde{\varphi}_{\alpha}\right\rangle \mid e_{\alpha} \in K-L\right\}$ é uma base para $C_{n}(\tilde{K}, \tilde{L})$ como um $\mathbb{Z} G$-módulo. (Referência: [5])

Assim, temos que $C(\tilde{K}, \tilde{L})$ é um $\mathbb{Z} G$-complexo livre.

Sejam $x \in K$ e $\tilde{x} \in p^{-1}(x)$ pontos fixados. Então existe um isomorfismo entre $G$ e $\pi_{1}(K, x)$ que é definido da seguinte forma: para cada caminho $\alpha:(I,\{0,1\}) \rightarrow(K, x)$, seja $\tilde{\alpha}$ o único levantamento de $\alpha$ com $\tilde{\alpha}(0)=\tilde{x}$; seja $g_{[\alpha]}: \tilde{K} \rightarrow \tilde{K}$ o único elemento de $G$ que satisfaz $g_{[\alpha]}(\tilde{x})=\tilde{\alpha}(1)$; então $\operatorname{definimos} \theta(x, \tilde{x})([\alpha])=g_{[\alpha]}$.

Temos que, se $y \in \tilde{K}$ e se $\omega:(I, 0,1) \rightarrow(\tilde{K}, \tilde{x}, y)$ é um caminho qualquer, então $g_{[\alpha]}(y)=\widetilde{\alpha * p \omega}(1)$.

Suponha que $p: \tilde{K} \rightarrow K$ e $p^{\prime}: \tilde{L} \rightarrow L$ são recobrimentos universais com $p(\tilde{x})=x$ e $p^{\prime}(\tilde{y})=y$. Sejam $G_{K}=\operatorname{Cov}(\tilde{K})$ e $G_{L}=\operatorname{Cov}(\tilde{L})$. Então qualquer aplicação $f:(K, x) \rightarrow(L, y)$ induz uma única aplicação $f_{\sharp}: G_{K} \rightarrow G_{L}$ tal que o diagrama a seguir comuta

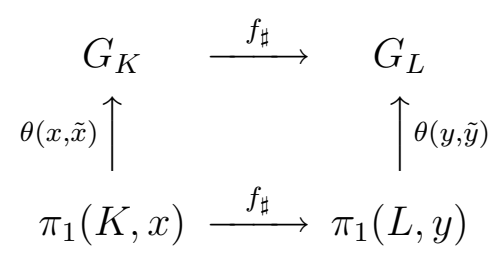

Proposição 1.17. Se $g \in G_{K}$ e se $\tilde{f}:(\tilde{K}, \tilde{x}) \rightarrow(\tilde{L}, \tilde{y})$ é um levantamento para $f$, então $f_{\sharp}(g) \circ \tilde{f}=\tilde{f} \circ g$. (Referência: [5]) 


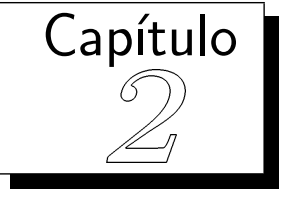

\section{Homotopia Simples}

\subsection{Deformações formais}

Apresentamos nesta seção os conceitos fundamentais da teoria de homotopia simples.

Definição 2.1. Seja $(K, L)$ um par CW finito, isto é, a família de células abertas de $K$ é finita. Então dizemos que $K$ colapsa para $L$ por um colapso elementar quando:

(i) $K=L \cup e^{n-1} \cup e^{n}$, onde $e^{n}$ e $e^{n-1}$ são células de $K$ que não estão em $L$,

(ii) existe uma aplicação $\varphi: I^{n} \rightarrow K$ tal que:

(a) $\varphi$ é uma aplicação característica para a célula $e^{n}$,

(b) $\varphi \mid I^{n-1}$ é uma aplicação característica para a célula $e^{n-1}$,

(c) $\varphi\left(J^{n-1}\right) \subset L^{n-1}$.

Neste caso, denotamos $K \searrow L$. Podemos dizer também que $L$ expande para $K$ por uma expansão elementar e então denotamos $L$ e $K$. 
Geometricamente, uma expansão elementar de $L$ corresponde à colagem de parte do bordo de uma $n$-bola em $L$ de maneira que a parte do bordo desta bola que não foi colada seja homeomorfa a uma $(n-1)$-bola aberta e a parte do bordo que foi colada a $L$ esteja contida em uma união de células de $L$ de dimensão no máximo $n-1$. Além disso, o fêcho da $(n-1)$-bola aberta que não foi colada deve estar contido em uma união de células de $L$ de dimensão no máximo $n-2$.

De fato, se escrevermos $\varphi_{0}=\varphi \mid J^{n-1}$ na definição acima teremos que

$$
(K, L) \cong\left(L \cup_{\varphi_{0}} I^{n}, L\right)
$$

Note que $\varphi_{0}\left(\partial J^{n-1}\right) \subset L^{n-2}$.

Por outro lado, dados um complexo CW finito $L$ e uma aplicação $\varphi_{0}$ : $\left(J^{n-1}, \partial J^{n-1}\right) \rightarrow\left(L^{n-1}, L^{n-2}\right)$, podemos construir um novo complexo CW $K=L \cup_{\varphi_{0}} I^{n}$ de maneira que $K \searrow L$

Proposição 2.1. Se $K \searrow L$ então

(i) Existe uma retração por deformação forte celular $D: K \rightarrow L$.

(ii) Quaisquer duas retrações por deformação forte de $K$ em $L$ são homotópicas relativamente a $L$.

Demonstração. Suponha que $K=L \cup e^{n-1} \cup e^{n}$. Seja $\varphi$ uma aplicação característica que satisfaz as condições da definição de colapso elementar. Como $\operatorname{vimos}(K, L) \cong\left(L \cup_{\varphi_{0}} I^{n}, L\right)$, onde $\varphi_{0}=\varphi \mid J^{n-1}$. Agora, $L \underset{\varphi_{0}}{\cup} I^{n}$ pode ser considerado como o cilindro da aplicação $\varphi_{0}$. Assim, por $(1.4)$, temos que $L \cup_{\varphi_{0}} I^{n} \smile_{\downarrow} L$, o que prova (i).

Sejam $D_{1}, D_{2}: K \rightarrow L$ duas retrações por deformação forte e $i: L \rightarrow K$ a aplicação inclusão. Então temos que $i D_{1} \simeq 1_{K} \simeq i D_{2}$ rel $L$, donde $D_{1}=$ $D_{1} i D_{1} \simeq D_{1} i D_{2}=D_{2}$ rel $L$.

Observe que na prova de (ii) utilizamos apenas o fato de $L<K$. 
Definição 2.2. (a) Dizemos que $K$ colapsa para $L$ ou $L$ expande para $K$ e denotamos $K \searrow L$ ou $L \nearrow K$, respectivamente, quando existe uma seqüência finita (possivelmente vazia) de colapsos elementares:

$$
K=K_{0} \searrow K_{1} \searrow \ldots \searrow K_{q}=L .
$$

(b) Uma seqüência finita $K=K_{0} \rightarrow K_{1} \rightarrow \cdots \rightarrow K_{q}=L$ de forma que cada seta indica ou um colapso elementar ou uma expansão elementar é chamada de deformação formal. Se existe uma deformação formal de $K$ em $L$, denotamos $K \wedge L$. Observe que, neste caso, também temos que $L \wedge K$.

(c) Quando existe uma deformação formal entre dois complexos dizemos que estes complexos têm o mesmo tipo de homotopia simples.

(d) Se $K$ e $L$ têm um subcomplexo $K_{0}$ em comum e $K \wedge L$ de maneira que durante a deformação formal nenhuma célula de $K_{0}$ é removida, escrevemos $K \wedge L$ rel $K_{0}$.

Seja $K=K_{0} \rightarrow K_{1} \rightarrow \cdots \rightarrow K_{q}=L$ uma deformação formal. Defina $f_{i}: K_{i} \rightarrow K_{i+1}$ como sendo a aplicação inclusão se a seta $K_{i} \rightarrow K_{i+1}$ na deformação formal acima indica uma expansão elementar ou uma retração por deformação forte celular qualquer se a seta indica um colapso elementar (note que o resultado (2.1) garante a existência de uma retração por deformação forte neste caso).

Definição 2.3. Nas condições acima chamamos a função $f=f_{q-1} \ldots f_{1} f_{0}$ de uma deformação.

Observe que, pela proposição (2.1), quaisquer duas deformações relacionadas a uma mesma deformação formal são homotópicas. Além disso, uma deformação é sempre uma equivalência de homotopia celular, uma vez que é a composta de funções com estas características.

Definição 2.4. Seja $K_{0}$ um subcomplexo de $K$ e seja $f=f_{q-1} \ldots f_{1} f_{0}$ : $K \rightarrow L$ uma deformação tal que $f_{i} \mid K_{0}=1, \forall i$. Então dizemos que $f$ é uma deformação rel a $K_{0}$. 
Note que nestas condições $K \wedge L$ rel $K_{0}$.

Definição 2.5. Definimos uma equivalência de homotopia simples $f: K \rightarrow$ $L$ como sendo uma aplicação homotópica a uma deformação. Dizemos que $f$ é uma equivalência de homotopia simples rel a $K_{0}$ se ela é homotópica rel $K_{0}$ a uma deformação rel $K_{0}$.

As seguintes conjecturas são naturais:

Conjectura (I) Se $f: K \rightarrow L$ é uma equivalência de homotopia, então $f$ é uma equivalência de homotopia simples.

Conjectura (II) Se existe uma equivalência de homotopia entre $K$ e $L$, então existe uma equivalência de homotopia simples entre estes dois complexos.

Mostraremos neste trabalho que em geral ambas as conjecturas acima são falsas, mas que em muitos casos particulares ambas as conjecturas são verdadeiras ou (I) é falsa enquanto que (II) é verdadeira.

\subsection{Cilindros de aplicação e deformações}

Nesta seção vamos obter uma reformulação mais conveniente para a conjectura (I) enunciada na seção anterior. Para obter esta reformulação usaremos alguns resultados que relacionam cilindros de aplicação e deformações formais. Estes resultados em geral são provados usando apenas como requisitos as definições dos objetos em questão, por isso suas demonstrações serão omitidas. O leitor mais exigente pode encontrá-las em [5].

Proposição 2.2. Sejam $f: K \rightarrow L$ uma aplicação celular e $K_{0}$ um subcomplexo de $K$, então temos que $M_{f} \searrow M_{f \mid K_{0}}$.

Corolário 2.3. Seja $f: K \rightarrow L$ uma aplicação celular, então $M_{f} \searrow L$.

Corolário 2.4. Sejam $K_{0}$ um subcomplexo de $K$ e $i=0$ ou 1 , então $(K \times$ $I) \searrow\left(K_{0} \times I\right) \cup(K \times i)$. 
Corolário 2.5. Sejam $K_{0}$ um subcomplexo de $K$ e vK o cone de $K$, então $v K \searrow v K_{0}$.

Lema 2.6. (i) Seja $\left(K, K_{1}, K_{2}\right)$ um trio que é $C W$ isomorfo ao trio $\left(J, J_{1}, J_{2}\right)$ tal que $K \wedge K_{1}$ rel $K_{2}$. Então $J \wedge J_{1}$ rel $J_{2}$.

(ii) Sejam $K_{1}, K_{2}$ e $L$ complexos $C W$ tais que $L$ é subcomplexo de $K_{1}$ e $K_{2}$ e seja $h: K_{1} \rightarrow K_{2}$ um isomorfismo $C W$ tal que $h \mid L=1$, então $K_{1} \wedge K_{2}$ rel $L$.

Devido a este resultado, quando for conveniente, substituiremos um determinado complexo CW por um outro CW isomorfo a este sem fazer qualquer comentário.

Proposição 2.7 (Princípio da relatividade). Sejam $J, K, L_{1}$ e $L_{2}$ complexos $C W$ tais que $L_{1}$ é subcomplexo de $J$ e de $K$. Seja $f: L_{1} \rightarrow L_{2}$ uma aplicação celular. Se $K \wedge J$ rel L, então $K \cup_{f} L_{2} \wedge L \cup_{f} L_{2}$ rel $L_{2}$.

Corolário 2.8. Sejam $K \cup L_{2}$ e $J \cup L_{2}$ dois complexos $C W$ que têm como subcomplexos $K, L_{2}$ e $J, L_{2}$, respectivamente. Suponha que $K \cap L_{2}=J \cap L_{2}=$ $L_{1}$ e que $K \wedge J$ rel $L_{1}$, então $K \cup L_{2} \wedge J \cup L_{2}$ rel $L_{2}$.

Proposição 2.9. Seja $f: K \rightarrow L$ uma aplicação celular. Suponha que $K \searrow K_{0}$, então $M_{f} \searrow K \cup M_{f \mid K_{0}}$.

Proposição 2.10. Sejam $f, g: K \rightarrow L$ aplicações celulares homotópicas, então $M_{f} \wedge M_{g}$ rel $K \cup L$.

Proposição 2.11. Seja $K_{1} \stackrel{f_{1}}{\longrightarrow} K_{2} \stackrel{f_{2}}{\longrightarrow} \cdots \stackrel{f_{q-1}}{\longrightarrow} K_{q}$ uma seqüência de aplicações celulares. Se $f=f_{q-1} \ldots f_{2} f_{1}$ então $M_{f} \searrow M_{f_{1}} \cup M_{f_{2}} \cup \cdots \cup$ $M_{f_{q-1}} \operatorname{rel}\left(K_{1} \cup K_{q}\right)$, onde esta união é disjunta de forma que cada $K_{i-1} \subset M_{f_{i}}$ é identificado trivialmente com $K_{i-1} \subset M_{f_{i-1}}$.

Teorema 2.12. Dada uma aplicação $f: K \rightarrow L$, as seguintes afirmações são equivalentes:

(i) f é uma equivalência de homotopia simples. 
(ii) Existe uma aproximação celular g para $f$ tal que $M_{g} \wedge K$ rel $K$.

(iii) Qualquer aproximação celular g para $f$ é tal que $M_{g} \wedge K$ rel $K$.

Teorema 2.13 (Teorema de extensão para a homotopia simples). Sejam $X, K_{0}$ e $K$ complexos $C W$ tais que $X<K_{0}<K$ e $f: K_{0} \rightarrow L_{0}$ uma equivalência de homotopia simples celular tal que $f \mid X=1$. Então existe uma equivalência de homotopia simples $F: K \rightarrow L$ tal que $F \mid K_{0}=f$, onde $L=K \cup_{f} L_{0}$. Além disso, $K \wedge L$ rel $X$.

Como mencionamos, estes resultados nos permitem obter uma nova formulação para a conjectura (I) dada por

Conjectura (I') Se $(X, Y)$ é um par CW e $X{ }^{\} Y$ então $X \wedge Y$ rel $Y$.

Mostremos a equivalência entre as duas conjecturas:

(I) $\Rightarrow\left(I^{\prime}\right)$

Como $X X Y$, existe uma retração por deformação forte $D: X \rightarrow Y$. Mas, toda retração por deformação forte é uma equivalência de homotopia, logo, por (I), $D$ é uma equivalência de homotopia simples. Assim, $X \wedge Y$. Agora, como $D \mid Y=1_{Y}$, temos que a deformação $X \wedge Y$ é rel a $Y$.

$\left(\mathrm{I}^{\prime}\right) \Rightarrow(\mathrm{I})$

Seja $f: K \rightarrow L$ uma equivalência de homotopia. Tome $g: K \rightarrow L$ uma aproximação celular para $f$. Então $g$ também é uma equivalência de homotopia. Segue de (1.8), que $M_{g} \ K$. Logo, por (I'), $M_{g} \wedge K$ rel $K$, e então o resultado 2.12 nos garante que $f$ é uma equivalência de homotopia simples.

\subsection{O grupo de Whitehead de um complexo CW}

Seja $L$ um complexo CW finito. Considere a classe de todos os pares CW $(K, L)$ tais que $K{ }^{\sim} L$. 
Nesta classe considere a relação:

$$
(K, L) \sim\left(K^{\prime}, L\right) \Longleftrightarrow K \wedge K^{\prime} \text { rel } L
$$

Facilmente verifica-se que esta relação é uma relação de equivalência.

Denotaremos a classe de equivalência de um par $(K, L)$ por $[K, L]$ e o conjunto destas classes de equivalências por $W h(L)$.

Neste conjunto podemos definir uma operação dada por:

$$
[K, L]+\left[K^{\prime}, L\right]=\left[K \cup_{L} K^{\prime}, L\right]
$$

onde $K \cup_{L} K^{\prime}$ é a união disjunta de $K$ e $K^{\prime}$ identificados pela aplicação identidade em $L$.

Verifiquemos a boa-definição desta operação:

Primeiramente observe que, como $K{ }_{\downarrow} L$ e $\left.K^{\prime}\right\urcorner_{\downarrow} L, K \cup_{L} K^{\prime} \bigcup_{\downarrow} L$.

Agora, verifiquemos que se $[K, L]=[J, L]$ e $\left[K^{\prime}, L\right]=\left[J^{\prime}, L\right]$, então $\left[J \cup_{L}\right.$ $\left.J^{\prime}, L\right]=\left[K \cup_{L} K^{\prime}, L\right]$.

Pelo princípio da relatividade, temos que $K \cup_{L} K^{\prime} \wedge J \cup_{L} K^{\prime}$ rel $L$ e que $J \cup_{L} K^{\prime} \wedge J \cup_{L} J^{\prime}$ rel $L$, donde $K \cup_{L} K^{\prime} \wedge J \cup_{L} J^{\prime}$ rel $L$, o que prova a afirmação anterior.

Teorema 2.14. Wh(L) com a operação definida acima é um grupo abeliano.

Demonstração. Sejam $K, K^{\prime}$ e $K^{\prime \prime}$ complexos CW que têm $L$ como subcomplexo e retrato de deformação forte.

Claramente, $\left(K \cup_{L} K^{\prime}\right) \cup_{L} K^{\prime \prime}$ é CW isomorfo a $K \cup_{L}\left(K^{\prime} \cup_{L} K^{\prime \prime}\right)$ por um isomorfismo $\mathrm{CW}$ que restrito a $L$ é a identidade. Assim, por (2.6)(ii), $\left(K \cup_{L} K^{\prime}\right) \cup_{L} K^{\prime \prime} \wedge K \cup_{L}\left(K^{\prime} \cup_{L} K^{\prime \prime}\right)$ rel $L$, e portanto a propriedade associativa é válida.

De maneira análoga, verificamos a propriedade comutativa.

A prova de que $[L, L]$ é o elemento neutro é trivial. 
Seja $D: K \rightarrow L$ uma retração por deformação forte celular. Defina o complexo $\mathrm{CW} 2 M_{D}$ como sendo o espaço $K \times[-1,1]$ com as identificações $(x,-1)=(D(x),-1)$ e $(x, 1)=D(x)$, para todo $x \in K$. Observe que $2 M_{D}$ consiste da união disjunta de $M_{D} \oplus M_{D}$ identificando estes espaços pela identidade em $K$. Temos que $M_{D}{ }^{2} L$ por 1.4 e que $M_{D}{ }^{2} K$ por 1.8 , seque que $2 M_{D}{ }^{2} L$.

Verifiquemos que $\left[2 M_{D}, L\right]+[K, L]=[L, L]$.

Sejam

$$
M_{D}^{\prime}=\frac{K \times[0,-1]}{(x,-1)=(D(x),-1)} \subset 2 M_{D}
$$

e $i: L \rightarrow K$ a aplicação inclusão.

Então,

$$
\begin{aligned}
{\left[2 M_{D}, L\right]+[K, L] } & =\left[2 M_{D} \cup_{L} K, L\right] \\
& =\left[\left(M_{D} \cup_{L} K\right) \cup M_{D}^{\prime}, L\right] \\
& =\left[M_{i D} \cup M_{D}^{\prime}, L\right] .
\end{aligned}
$$

Agora, veja que $i D$ é homotópica a aplicação $1_{K}\left(\right.$ escrevemos $\left.i D \simeq 1_{K}\right)$, donde, por 2.10), $M_{i D} \wedge M_{1_{K}}$ rel $(K \times 0) \cup K$. (Observe que $M_{1_{K}}=K \times I$.)

Assim,

$$
\begin{aligned}
{\left[M_{i D} \cup M_{D}^{\prime}, L\right] } & =\left[(K \times I) \cup M_{D}^{\prime}, L\right] \\
\stackrel{2.4}{=} & {\left[(L \times I) \cup M_{D}^{\prime}, L\right] } \\
\stackrel{2.2}{=} & {[L \times[-1,1], L] } \\
= & {[L, L]=0, }
\end{aligned}
$$

o que completa a prova.

Definição 2.6. Chamamos $W h(L)$ de grupo de Whitehead de $L$.

Sejam $L_{1}$ e $L_{2}$ complexos CW finitos e $f: L_{1} \rightarrow L_{2}$ uma aplicação celular. Esta função induz um homomorfismo de grupos $f_{*}: W h\left(L_{1}\right) \rightarrow W h\left(L_{2}\right)$ dado 
por:

$$
f_{*}\left(\left[K, L_{1}\right]\right)=\left[K \cup_{L_{1}} M_{f}, L_{2}\right]
$$

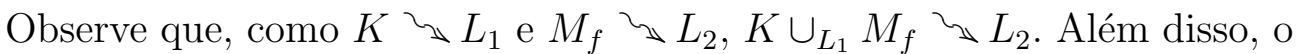
princípio da relatividade nos garante a boa-definição de $f_{*}$.

Esta função é equivalentemente definida por:

$$
f_{*}\left(\left[K, L_{1}\right]\right)=\left[K \underset{f}{\cup} L_{2}, L_{2}\right]
$$

De fato, pelo corolário (2.3), $M_{f} \searrow L_{2}$, e então os resultados (2.1) e (1.4), nos garantem que $p: M_{f} \rightarrow L_{2}$ é uma equivalência de homotopia simples celular.

Agora, observe que $p \mid L_{2}=1$, logo o teorema (2.13) nos fornece que

$$
\left(M_{f} \cup_{L_{1}} K\right) \wedge\left(M_{f} \cup_{L_{1}} K\right) \cup_{p} L_{2}=K \cup_{f} L_{2} \text { rel } L_{2} .
$$

Com esta definição, é trivial mostrar que $f_{*}$ é um homomorfismo de grupos.

Mostra-se também sem dificuldades que $g_{*} f_{*}=(g f)_{*}$.

Assim, temos que existe um funtor covariante da categoria dos complexos CW finitos e aplicações celulares na categoria dos grupos abelianos e homomorfismos de grupos. Este funtor faz as seguintes associações:

$$
\begin{gathered}
L \mapsto W h(L) \\
\left(f: L_{1} \rightarrow L_{2}\right) \mapsto\left(f_{*}: W h\left(L_{1}\right) \rightarrow W h\left(L_{2}\right)\right) .
\end{gathered}
$$

Proposição 2.15. Sejam $f, g: L_{1} \rightarrow L_{2}$ duas aplicações celulares entre complexos finitos tais que $f \simeq g$. Então $f_{*}=g_{*}$.

Demonstração. É imediata da primeira definição para $f_{*}$ e $g_{*}$ e da proposição (2.10). 
Definição 2.7. Definimos a torção de uma equivalência de homotopia celular entre complexos finitos $f: L_{1} \rightarrow L_{2}$ e indicamos por $\tau(f)$, o elemento $\tau(f)=$ $f_{*}\left(\left[M_{f}, L_{1}\right]\right)$ de $W h\left(L_{2}\right)$.

Observe que como $f$ é uma equivalência de homotopia $M_{f} \longleftarrow_{\checkmark} L_{1}$. Além disso, $M_{f}$ é um complexo CW, pois $f$ é uma aplicação celular, logo a definição acima faz sentido.

\subsection{Simplificação de pares CW homotopicamente triviais}

Nesta seção, a partir de um par CW $(K, L)$ tal que $K{ }_{\downarrow} L$, vamos obter um outro par CW $(J, L)$, que também satisfaz $J{ }^{2} L$, de forma que ambos os pares representem a mesma classe de equivalência em $W h(L)$, mas de maneira que $J$ seja um complexo mais simples.

Daqui em diante $L$ sempre representará um complexo CW finito.

Lema 2.16. Sejam $K_{0}=L \cup e_{0}$ e $K_{1}=L \cup e_{1}$ dois complexos $C W$. Suponha que $e_{i}(i=0,1)$ são $n$-células que têm aplicações características $\varphi_{i}: I^{n} \rightarrow$ $K_{i}$ tais que $\varphi_{0} \mid \partial I^{n}$ e $\varphi_{1} \mid \partial I^{n}$ são aplicações homotópicas em L, então $K_{0} \wedge K_{1}$ rel L. (Refrência: [5])

Teorema 2.17. Seja $(K, L)$ um par de complexos $C W$ conexos. Suponha que $r$ é um inteiro satisfazendo as seguintes condições:

(i) $\pi_{r}(K, L)=0$,

(ii) $K=L \cup \bigcup_{i=1}^{k_{r}} e_{i}^{r} \cup \bigcup_{i=1}^{k_{r+1}} e_{i}^{r+1} \cup \cdots \cup \bigcup_{i=1}^{k_{n}} e_{i}^{n}$.

Então existe um complexo $C W M$ da forma

$$
M=L \cup \bigcup_{i=1}^{k_{r+1}} f_{i}^{r+1} \cup \bigcup_{i=1}^{k_{r}+k_{r+2}} f_{i}^{r+2} \cup\left(\bigcup_{i=1}^{k_{r+3}} f_{i}^{r+3} \cup \cdots \cup \bigcup_{i=1}^{k_{n}} f_{i}^{n}\right)
$$

tal que $K \wedge M$ rel L. (Aqui $e_{i}^{j}$ e $f_{i}^{j}$ denotam j-células.) 
Demonstração. Provaremos este teorema para o caso específico em que $k_{r}=$ 1. A prova para o caso em que $k_{r}$ é um natural qualquer é apenas uma generalização do que faremos.

Então seja $e^{r}$ a única $r$-célula em $K-L$. Fixe uma aplicação característica $\varphi: I^{r} \rightarrow K$ para esta célula. Temos que $\varphi\left(\partial I^{r}\right) \subset K^{r-1}=L^{r-1}$. Como $\pi_{r}(K, L)=0$, temos que existe uma homotopia $F: I^{r} \times I \rightarrow K$ tal que

$$
\begin{aligned}
& F_{0}=\varphi \\
& F_{t}\left|\partial I^{r}=\varphi\right| \partial I^{r}, \forall t \in I \\
& F_{1}\left(I^{r}\right) \subset L .
\end{aligned}
$$

Podemos assumir que $F$ também satisfaz as condições

$$
\begin{aligned}
& F\left(\partial I^{r+1}\right) \subset K^{r} \\
& F\left(I^{r+1}\right) \subset K^{r+1}
\end{aligned}
$$

Isto porque, caso contrário, podemos obter, aplicando os teoremas da aproximação celular e o de extensão por homotopia, uma outra homotopia que satisfaça todas as condições acima.

Seja $P=K \underset{F}{\cup} I^{r+2}$. Denote por $\psi: I^{r+2} \rightarrow P$ a aplicação identificação. Então $\psi \mid I^{r+1} \times 0=F$.

Escreva $E^{r+2}=\psi(\stackrel{\circ}{r+2})$ e $E^{r+1}=\psi(\stackrel{\circ}{r+1})$.

Logo, podemos escrever $P=K \cup E^{r+1} \cup E^{r+2}$. Desta forma, fica claro que $P \searrow K$.

Seja $P_{0}=L \cup e^{r} \cup E^{r+1}$. Como $\psi\left(\partial J^{r+1}\right)=F\left(\partial I^{r+1}\right) \subset K^{r} \subset L \cup e^{r}, P_{0}$ é um subcomplexo de $P$ bem definido.

Temos que $\psi \mid J^{r+1}: J^{r+1} \rightarrow P_{0}$ é uma aplicação característica para $E^{r+1}$ tal que $\psi \mid I^{r}$ é uma aplicação característica para $e^{r}$ e $\psi\left(\partial J^{r+1}-I^{r}\right)=$ $F\left(\partial I^{r+1}-I^{r}\right) \subset L^{r}, \operatorname{logo} P_{0} \searrow L$. Seja $g: P_{0} \rightarrow L$ uma deformação celular correspondente a este colapso.

Então podemos aplicar o teorema de extensão para a homotopia simples de forma a obter que $K$ e $P \wedge P \cup \underset{g}{\cup} L$ rel $L$. 
Por fim, observe que $M=P \cup_{g} L$ tem a forma do enunciado.

Teorema 2.18. Seja $(K, L)$ um par de complexos $C W$ conexos homotopicamente trivial. Sejam $n=\operatorname{dim}(K-L), r \geq n-1, r \in \mathbb{Z}$ e e uma 0-célula qualquer de L. Então $K \wedge M$ rel L, onde

$$
M=L \cup \bigcup_{j=1}^{a} e_{j}^{r} \cup \bigcup_{i=1}^{a} e_{i}^{r+1}
$$

e as células $e_{j}^{r}$ e $e_{i}^{r+1}$ têm aplicações características $\psi_{j}: I^{r} \rightarrow M$ e $\varphi_{i}$ : $I^{r+1} \rightarrow M$, respectivamente, de forma que $\psi_{j}\left(\partial I^{r}\right)=e^{0}=\varphi_{i}\left(J^{r}\right)$.

Demonstração. Como $K{ }_{\downarrow} L$, o teorema 1.2 , nos garante que $\pi_{i}(K, L)=$ $0, \forall i$. Assim, podemos aplicar o resultado 2.17) para obter um complexo $K_{0}$ tal que $K_{0} \wedge K$ rel $L$ e $K_{0}-L$ não tem 0 -células e tem $m$ 2-células, onde $m$ é a soma do número de 0-células com o número de 2-células de $K-L$.

O complexo $K_{0}$ também satisfaz as condições de (2.17), portanto podemos aplicar novamente este resultado de forma a obter um outro complexo $K_{1}$ tal que $K_{1} \wedge K_{0}$ rel $L$ e que, então, $K_{1} \wedge K$ rel $L$ e de maneira que o número de 3-células de $K_{1}-L$ é a soma do número de 1-células com o número de 3-células de $K_{0}-L$ e o número de 1-células é zero, assim como o número de 0-células.

Podemos repetir este procedimento um número finito de vezes de forma a obter um complexo $\hat{K}$ tal que $\hat{K} \wedge K$ rel $L$ e $\hat{K}-L$ tem apenas células de dimensão $r$ e $r+1(\operatorname{com} r \geq n-1)$.

Então podemos escrever

$$
\hat{K}=L \cup \bigcup_{j=1}^{a} \hat{e}_{j}^{r} \cup \bigcup_{i=1}^{b} \hat{e}_{i}^{r+1} .
$$

Tome uma aplicação característica $\hat{\psi}_{j}: I^{r} \rightarrow \hat{K}$ para cada célula $\hat{e}_{j}^{r}$.

Como $\hat{K} \ L$, existe uma retração $R: \hat{K} \rightarrow L$, então temos que $R \hat{\psi}_{j}$ : $I^{r} \rightarrow L$ e $R \hat{\psi}_{j}\left|\partial I^{r}=\hat{\psi}_{j}\right| \partial I^{r}\left(\right.$ pois $\left.\hat{\psi}_{j}\left(\partial I^{r}\right) \subset L\right)$. Segue que $\hat{\psi}_{j} \mid \partial I^{r}$ é 
homotópica em $L$ a uma aplicação constante e, como $L$ é conexo por caminhos (pois todo complexo CW conexo é conexo por caminhos), é homotópica em $L$ à aplicação constante $\partial I^{r} \rightarrow e^{0}$.

Observe que como $j$ foi tomado de forma arbitrária as informações que obtemos acima são válidas para todo $j=1, \ldots, a$.

Assim, podemos aplicar o lema (2.16), para obter que

$$
L \cup \bigcup_{j=1}^{a} \hat{e}_{j}^{r} \wedge L \cup \bigcup_{j=1}^{a} e_{j}^{r} \text { rel } L,
$$

onde as células $e_{j}^{r}$ são coladas trivialmente em $e^{0}$.

Então o teorema de extensão para a homotopia simples nos fornece que

$$
L \cup \bigcup_{j=1}^{a} \hat{e}_{j}^{r} \cup \bigcup_{i=1}^{b} \hat{e}_{i}^{r+1} \wedge L \cup \bigcup_{j=1}^{a} e_{j}^{r} \cup \bigcup_{i=1}^{b} f_{i}^{r+1} \text { rel } L .
$$

Para cada $i$, seja $\hat{\varphi}_{i}: I^{r+1} \rightarrow L \cup \bigcup_{j=1}^{a} e_{j}^{r}$ uma aplicação característica para $f_{i}^{r+1}$. Temos que cada $\hat{\varphi}_{i} \mid \partial I^{r+1}$ é homotópica a uma aplicação $\varphi_{i}: \partial I^{r+1} \rightarrow$ $L \cup \bigcup_{j=1}^{a} e_{j}^{r}$ que satisfaz $\varphi_{i}\left(J^{r}\right)=e^{0}$ (pois $J^{r}$ é contrátil a um ponto em $\partial I^{r+1}$ ).

Logo, aplicando o lema 2.16 novamente, obtemos que

$$
L \cup \bigcup_{j=1}^{a} e_{j}^{r} \cup \bigcup_{i=1}^{b} f_{i}^{r+1} \wedge L \cup \bigcup_{j=1}^{a} e_{j}^{r} \cup \bigcup_{i=1}^{b} e_{i}^{r+1} \text { rel } L
$$

onde cada $e_{i}^{r+1}$ tem aplicação colagem $\varphi_{i}$.

Defina $M=L \cup \bigcup_{j=1}^{a} e_{j}^{r} \cup \bigcup_{i=1}^{b} e_{i}^{r+1}$.

Verifiquemos que $a=b$.

Observe que, por (1.9), o número de $r$-células (resp., $(r+1)$-células) de $M-L$ é igual ao rank de $H_{r}\left(M^{r} \cup L, L\right)$ (resp., $H_{r+1}\left(M, M^{r} \cup L\right)$ ). Mas, como $M{ }^{L} L, H_{r+1}(M, L)=H_{r}(K, L)=0$. Segue que a seqüência exata do trio $\left(M, M^{r} \cup L, L\right)$ contém

$$
\cdots \rightarrow 0 \rightarrow H_{r+1}\left(M, M^{r} \cup L\right) \stackrel{d}{\longrightarrow} H_{r}\left(M^{r} \cup L, L\right) \rightarrow 0 \rightarrow \ldots,
$$


donde $d$ é um isomorfismo e então $H_{r}\left(M^{r} \cup L, L\right)$ e $H_{r+1}\left(M, M^{r} \cup L\right)$ possuem o mesmo rank.

Assim, $M$ é o complexo procurado.

Definição 2.8. Se $M$ é um complexo $\mathrm{CW}$ tal que $M$ \ $L$ e o par $(M, L)$ satisfaz a conclusão do teorema acima com $r \geq 2$, então dizemos que o par $(M, L)$ está na forma simplificada.

\subsection{Matrizes e deformações formais}

Nesta seção associaremos a cada par $(K, L)$ na forma simplificada, uma matriz com entradas no anel $\mathbb{Z}\left(\pi_{1}\left(L, e^{0}\right)\right)$, onde $e^{0}$ é uma 0-célula de $L$. Em seguida, vamos obter alguns resultados que serão úteis no decorrer deste trabalho.

Dado um par de complexos CW conexos $\left(P, P_{0}\right)$, fixemos um ponto $x \in$ $P_{0}$.

Consideremos a ação de $\pi_{1}=\pi_{1}\left(P_{0} ; x\right)$ sobre $\pi_{n}\left(P, P_{0} ; x\right)$ dada por $[\alpha] \cdot[\varphi]=\left[\varphi^{\alpha}\right]$, onde $\alpha$ e $\varphi$ são representantes dos elementos $[\alpha]$ e $[\varphi]$ de $\pi_{1}$ e $\pi_{n}\left(P, P_{0} ; x\right)$, respectivamente, e a função $\varphi^{\alpha}:\left(I^{n}, I^{n-1}, J^{n-1}\right) \rightarrow$ $\left(P, P_{0}, x\right)$ é obtida de forma a ser homotópica a $\varphi$ por uma homotopia que "arrasta" $\varphi\left(J^{n-1}\right)$ ao longo do laço $\alpha^{-1}$.

Trata-se realmente de uma ação bem-definida como pode ser checado em [13], por exemplo.

As seguintes propriedades são válidas:

(i) $[*] \cdot[\varphi]=[\varphi]$, onde $[*]$ indica o elemento identidade em $\pi_{1}$.

(ii) $[\alpha] \cdot\left(\left[\varphi_{1}\right]+\left[\varphi_{2}\right]\right)=[\alpha] \cdot\left[\varphi_{1}\right]+[\alpha] \cdot\left[\varphi_{2}\right]$.

(iii) $([\alpha][\beta]) \cdot[\varphi]=[\alpha] \cdot([\beta]) \cdot[\varphi])$. 
(iv) Esta ação comuta com todos os homomorfismos da seqüência exata de homotopia do par $\left(P, P_{0}\right)$.

Segue diretamente do que vimos acima que $\pi_{n}\left(P, P_{0} ; x\right)$ é um $\mathbb{Z} \pi_{1}$-módulo se considerarmos a multiplicação:

$$
\left(\sum n_{j}\left[\alpha_{j}\right]\right)[\varphi]=\sum n_{j}\left(\left[\alpha_{j}\right] \cdot[\varphi]\right)
$$

onde $n_{j} \in \mathbb{Z},\left[\alpha_{j}\right] \in \pi_{1}, \forall j$ e $[\varphi] \in \pi_{n}\left(P, P_{0} ; x\right)$.

Com esta multiplicação, a seqüência exata de homotopia de $\left(P, P_{0}, x\right)$ torna-se uma seqüência exata de $\mathbb{Z} \pi_{1}$-módulos.

Lema 2.19. Seja $\left(P, P_{0}\right)$ um par $C W \operatorname{com} P=P_{0} \cup \bigcup_{i=1}^{a} e_{i}^{n}$ e $P_{0}$ conexo. Suponha que $\varphi_{i}:\left(I^{n}, I^{n-1}, J^{n-1}\right) \rightarrow\left(P, P_{0}, e^{0}\right)$ são aplicaçôes características para as células $e_{i}^{n}$ e que ou $n \geq 3$, ou $n=2$ e $\varphi_{i}\left(\partial I^{n}\right)=e^{0}$ para todo $i$. Então $\pi_{n}\left(P, P_{0} ; e^{0}\right)$ é um $\mathbb{Z} \pi_{1}$-módulo livre com base $\left[\varphi_{1}\right],\left[\varphi_{2}\right], \ldots,\left[\varphi_{a}\right]$. (Referência: [5])

Considere agora o par na forma simplificada $(K, L)$ com

$$
K=L \cup \bigcup_{j=1}^{a} e_{j}^{r} \cup \bigcup_{i=1}^{a} e_{i}^{r+1}
$$

Sejam $\left\{\varphi_{i}\right\}_{i}$ e $\left\{\psi_{j}\right\}_{j}$ as aplicações características para as células $e_{i}^{r+1}$ e $e_{j}^{r}$, respectivamente, que satisfazem as condições do teorema (2.18).

Denotaremos $K_{r}=L \cup \bigcup_{j=1}^{a} e_{j}^{r}$.

Então, pelo lema precedente, temos que $\left\{\left[\varphi_{i}\right]\right\}_{i}$ e $\left\{\left[\psi_{j}\right]\right\}_{j}$ são bases para os $\mathbb{Z} \pi_{1}$-módulos $\pi_{r+1}\left(K, K_{r} ; e^{0}\right)$ e $\pi_{r}\left(K, L ; e^{0}\right)$, respectivamente.

Seja $\partial: \pi_{r+1}\left(K, K_{r} ; e^{0}\right) \rightarrow \pi_{r}\left(K, L ; e^{0}\right)$ o operador bordo da seqüência exata de homotopia do trio $\left(K, K_{r}, L\right)$.

Observe que, pelos comentários feitos nesta seção, este operador é um homomorfismo de $\mathbb{Z} \pi_{1}$-módulos. 
Definição 2.9. Definimos a matriz do par simplificado $(K, L)$ em relação às aplicações características $\left\{\varphi_{i}\right\}_{i}$ e $\left\{\psi_{j}\right\}_{j}$ como sendo a $a \times a \mathbb{Z} \pi_{1}$-matriz $\left(a_{i j}\right)$, onde $\partial\left[\varphi_{i}\right]=\sum a_{i j}\left[\psi_{j}\right]$.

Esta matriz é não-singular, ou seja, tem inversa dos dois lados.

Com efeito, como $K{ }_{\downarrow} L$, temos que $\pi_{r+1}(K, L)=\pi_{r}(K, L)=0$, logo, pela exatidão da seqüência de homotopia, $\partial$ é um isomorfismo.

Teorema 2.20. Seja $(K, L)$ um par na forma simplificada. Suponha que existem aplicações características $\left\{\varphi_{i}\right\}_{i}$ e $\left\{\psi_{j}\right\}_{j}$ de maneira que a matriz deste par em relação a estas aplicações seja a matriz identidade. Então K^LrelL. (Referência: [5])

Teorema 2.21. Seja $(K, L)$ um par na forma simplificada com matriz $\left(a_{i j}\right)$ em relação a algum conjunto de aplicações características. Suponha que a matriz $\left(a_{i j}\right)$ pode ser transformada numa outra matriz $\left(b_{i j}\right)$ através das seguintes transformações:

I. Multiplicar à esquerda uma linha da matriz por mais ou menos um elemento do grupo $\pi_{1}$ visto como subconjunto do anel $\mathbb{Z} \pi_{1}$. ( $R_{i} \rightarrow \pm \alpha R_{i}$, com $\alpha \in \pi_{1} \subset \mathbb{Z} \pi_{1}$.)

II. Adicionar a uma linha o múltiplo de uma outra linha da matriz por um elemento de $\mathbb{Z} \pi_{1}$, sendo que este elemento é multiplicado à esquerda. $\left(R_{k} \rightarrow R_{k}+\rho R_{i}\right.$, com $\rho \in \mathbb{Z} \pi_{1}$ e $k \neq i$.)

III. Expandir a matriz da seguinte forma:

$$
\left(a_{i j}\right) \rightarrow\left(\begin{array}{cc}
a_{i j} & 0 \\
0 & I_{q}
\end{array}\right)
$$

onde $I_{q}$ indica a $\mathbb{Z} \pi_{1}$-matriz identidade de ordem $q$.

Então existem um par na forma simplificada $(M, L)$ tal que $K \wedge M$ rel $L$ e um conjunto de aplicações características de maneira que $(M, L)$ tem matriz $\left(b_{i j}\right)$ com relação a estas aplicações. (Referência: [5])

Teorema 2.22. Seja $(K, L)$ um par na forma simplificada que tem matriz A com relação a algum conjunto de aplicações características. Suponha 
que a matriz A pode ser transformada em uma matriz identidade através de operações do tipo (I)-(V), onde (I), (II) e (III) são as operações descritas no teorema anterior $e$

IV. Multiplicar à direita uma coluna da matriz por mais ou menos um elemento de $\pi_{1}$. $\left(C_{j} \rightarrow \pm C_{j} \alpha\right.$, com $\alpha \in \pi_{1} \subset \mathbb{Z} \pi_{1}$.)

V. Adicionar a uma coluna da matriz o múltiplo de uma outra coluna por um elemento de $\mathbb{Z} \pi_{1}$, sendo que este elemento é multiplicado à direita. $\left(C_{k} \rightarrow C_{k}+C_{i} \rho\right.$, com $\rho \in \mathbb{Z}\left(\pi_{1}\right)$ e $k \neq i$.)

Então K^LrelL. (Referência: [5])

Teorema 2.23. Se $(K, L)$ é um par $C W$ tal que $K$ e $L$ são 1-conexos e $K \smile_{\downarrow} L$, então $K \wedge L$ rel $L$.

Demonstração. Por 2.18), existe um complexo $J$ tal que $(J, L)$ está na forma simplificada e $K \wedge J$ rel $L$. Seja $A$ a matriz de $(J, L)$ com respeito a algum conjunto de aplicações características. Como $\pi_{1} L=\{1\}, \mathbb{Z} \pi_{1}=\mathbb{Z}$. Então $A$ é uma matriz não-singular de coeficientes inteiros. Mas então $A$ pode ser transformada na matriz identidade por operações dos tipos I, II, IV e V. Segue que $J \wedge L$ rel $L$.

Seja $G$ um grupo. Chamamos de unidade de $\mathbb{Z} G$ um elemento que tem inverso multiplicativo de ambos os lados.

Facilmente, vemos que o conjunto $\pm G=\{g \mid g \in G\} \cup\{-g \mid g \in G\} \subset$ $\mathbb{Z} G$ é um grupo com a operação de multiplicação igual a do anel $\mathbb{Z} G$; assim, os elementos deste conjunto são unidades de $\mathbb{Z} G$; estes elementos são chamados de unidades triviais e o restante das unidades são chamadas de unidades não-triviais.

Teorema 2.24. Seja $G$ um grupo abeliano tal que $\mathbb{Z} G$ possui unidades nãotriviais. Então existe uma $\mathbb{Z} G$-matriz não-singular $A$ que não pode ser transformada na matriz identidade através de operações (I)-(V). 
$\mathbb{Z}_{5}$ é um exemplo de grupo abeliano tal que $\mathbb{Z}\left(\mathbb{Z}_{5}\right)$ possui unidades nãotriviais. De fato, se considerarmos $\mathbb{Z}_{5}=\left\{1, t, t^{2}, t^{3}, t^{4}\right\}$ então o elemento $1-t+t^{2} \in \mathbb{Z}\left(\mathbb{Z}_{5}\right)$ é uma das unidades não-triviais, pois $\left(1-t+t^{2}\right)\left(t+t^{2}-t^{4}\right)=$ 1 .

Teorema 2.25. Sejam $G$ um grupo finitamente apresentado e $A$ uma $\mathbb{Z} G$ matriz não-singular. Então

(i) Existe um complexo $C W$ conexo $L$ tal que $\pi_{1}\left(L, e^{0}\right)=G$.

(ii) Para qualquer complexo $L$ com $\pi_{1}\left(L, e^{0}\right)=G$, existe um outro complexo $C W K$, tal que $(K, L)$ está na forma simplificada e tem matriz A com respeito a algum conjunto de aplicações características.

(Referência: [5]) 


\section{Capítulo}

\section{Álgebra}

\subsection{Convenções}

Nesta seção faremos algumas convenções e enunciaremos alguns resultados que serão utilizados durante todo este capítulo.

Convenção 2. $R$ denotará um anel com unidade que satisfaz a seguinte propriedade:

(*) Se $M$ é um $R$-módulo livre finitamente gerado, então quaisquer duas de suas bases têm o mesmo número de elementos.

Convenção 3. Assumiremos que todo módulo é módulo à esquerda e finitamente gerado.

Note que, com estas duas convenções, temos que um $R$-módulo livre é um $R$-módulo com bases finitas, onde quaisquer duas destas bases têm a mesma cardinalidade.

Proposição 3.1. A condição $\left(^{*}\right)$ é satisfeita pelo anel $R$ se existe um anel de divisão $D$ e um homomorfismo de anéis não-nulo $f: R \rightarrow D$. 
Proposição 3.2. $\mathbb{Z} G$ satisfaz $\left(^{*}\right)$ para todo grupo $G$.

Demonstração. Seja $G$ um grupo qualquer.

A aplicação $A: \mathbb{Z} G \rightarrow \mathbb{Q}$, dada por $A\left(\sum_{i} n_{i} g_{i}\right)=\sum_{i} n_{i}$ é um homomorfismo de anéis não-nulo e $\mathbb{Q}$ é um anel de divisão. Portanto, o resultado anterior nos garante que $\mathbb{Z} G$ satisfaz $\left({ }^{*}\right)$.

Convenção 4. Sejam $M_{1}$ e $M_{2}$ módulos com bases $x=\left\{x_{1}, \ldots, x_{p}\right\}$ e $y=$ $\left\{y_{1}, \ldots, y_{q}\right\}$, respectivamente, e seja $f: M_{1} \rightarrow M_{2}$ um homomorfismo de módulos. Denotaremos por $\langle f\rangle_{x, y}$ a matriz $\left(a_{i j}\right)$, onde $f\left(x_{i}\right)=\sum_{j} a_{i j} y_{j}$. Quando não houver dúvidas sobre as bases em questão, denotaremos $\langle f\rangle_{x, y}$ simplesmente por $\langle f\rangle$.

É trivial provar que, com esta convenção, $\left\langle f_{2} f_{1}\right\rangle=\left\langle f_{1}\right\rangle\left\langle f_{2}\right\rangle$.

Convenção 5. Sejam $x=\left\{x_{1}, \ldots, x_{p}\right\}$ e $y=\left\{y_{1}, \ldots, y_{p}\right\}$ duas base para o $R$-módulo $M$, então denotaremos por $\langle x / y\rangle$ a matriz $\left(a_{i j}\right)$ dada por $x_{i}=$ $\sum_{j} a_{i j} y_{j}$

Note que as matrizes desta forma são não-singulares, pois $\langle x / y\rangle^{-1}=$ $\langle y / x\rangle$. Além disso, facilmente mostra-se que, se $z$ é uma outra base para $M$, então $\langle x / z\rangle=\langle x / y\rangle\langle y / z\rangle$.

Proposição 3.3. Sejam $M_{1} R$-módulo com bases $x$ e $x^{\prime}, M_{2} R$-módulo com bases y e $y^{\prime}$ e $f: M_{1} \rightarrow M_{2}$ um homomorfismo de módulos. Então vale

$$
\langle f\rangle_{x^{\prime}, y^{\prime}}=\left\langle x^{\prime} / x\right\rangle\langle f\rangle_{x, y}\left\langle y / y^{\prime}\right\rangle \text {. }
$$

\subsection{Os grupos $K_{G}(R)$}

Definição 3.1. Chamamos de $n$-ésimo grupo geral linear de $R$ e denotamos por $G L(n, R)$, o grupo das matrizes $n \times n$ com entradas em $R$ que possuem inversa dos dois lados (matrizes não-singulares). 
Existe uma aplicação injetiva natural de $G L(n, R)$ em $G L(n+1, R)$ que associa à cada matriz $A$, a matriz $\left(\begin{array}{cc}A & 0 \\ 0 & 1\end{array}\right)$. Usando este fato podemos definir: Definição 3.2. Chamamos de grupo geral linear infinito de $R$ o limite direto $\lim G L(n, R)$. Denotaremos este grupo por $G L(R)$.

Claramente, o grupo $G L(R)$ é constituído de todas as matrizes infinitas não-singulares que são a partir de certo ponto a identidade.

Por conveniência identificaremos cada $A \in G L(n, R)$ com sua imagem em $G L(R)$.

Denote por $E_{i, j}^{n}$ a matriz $n \times n$ que tem uma única entrada não-nula igual a 1 na posição $(i, j)$.

Definição 3.3. Definimos uma matriz elementar como sendo uma matriz da forma $\left(I_{n}+a E_{i, j}^{n}\right)$, com $i \neq j$ e $a \in R$.

Note que toda matriz elementar é não-singular (a saber $\left(I_{n}+a E_{i, j}^{n}\right)^{-1}=$ $\left.\left(I_{n}-a E_{i, j}^{n}\right)\right)$.

Denotaremos por $E(R)$ o subgrupo de $G L(R)$ gerado pelas matrizes elementares. Assim, os elementos de $E(R)$ são produtos finitos de matrizes elementares.

Considere a relação de equivalência em $G L(R)$ dada por:

$$
A \sim B \Longleftrightarrow \exists E_{1}, E_{2} \in E(R) \mid A=E_{1} B E_{2} .
$$

Equivalentemente, $A \sim B$ se e somente se $B$ pode ser transformada em $A$ através de uma seqüência finita das operações II, III e V definidas na última seção do capítulo anterior.

Proposição 3.4. Sejam A uma matriz $n \times n$ não-singular, $P_{1}$ submatriz $p \times n$ de $A, P_{2}$ submatriz $q \times n$ de $A$, ambas disjuntas entre si, e $X$ uma matriz $p \times q$ qualquer. Então valem: 


$$
\begin{aligned}
I_{R}: \quad A & =\left(\begin{array}{l}
\ddots \\
P_{1} \\
\ddots \\
P_{2} \\
\ddots
\end{array}\right) \sim B=\left(\begin{array}{c}
\ddots \\
P_{1}+X P_{2} \\
\ddots \\
P_{2} \\
\ddots
\end{array}\right) \\
I I_{R}: \quad A & =\left(\begin{array}{c}
\ddots \\
P_{1} \\
\ddots \\
P_{2} \\
\ddots
\end{array}\right) \sim B=\left(\begin{array}{c}
\ddots \\
P_{2} \\
\ddots \\
-P_{1} \\
\ddots
\end{array}\right), \text { para } p=q .
\end{aligned}
$$

No caso de $P_{1}$ ser uma submatriz $n \times p, P_{2}$ ser uma submatriz $n \times q$, ambas disjuntas entre si, e $X$ ser uma matriz $q \times p$ qualquer, então valem:

$I_{C}: \quad A=\left(\begin{array}{lllll}\ddots & P_{1} & \ddots & P_{2} & \ddots\end{array}\right) \sim B=\left(\begin{array}{lllll}\ddots & P_{1}+P_{2} X & \ddots & P_{2} & \ddots\end{array}\right)$

$I I_{C}: \quad A=\left(\begin{array}{lllll}\ddots & P_{1} & \ddots & P_{2} & \ddots\end{array}\right) \sim B=\left(\begin{array}{lllll}\ddots & -P_{2} & \ddots & P_{1} & \ddots\end{array}\right)$, para $p=q$.

Demonstração. $I_{R}$ segue trivialmente de um número finito de operações elementares.

$I I_{R}$ é provado a partir de $I_{R}$ da seguinte maneira:

$$
\left(\begin{array}{c}
\ddots \\
P_{1} \\
\ddots \\
P_{2} \\
\ddots
\end{array}\right) \sim\left(\begin{array}{c}
\ddots \\
P_{1}+P_{2} \\
\ddots \\
P_{2} \\
\ddots
\end{array}\right) \sim\left(\begin{array}{c}
\ddots \\
P_{1}+P_{2} \\
\ddots \\
-P_{1} \\
\ddots
\end{array}\right) \sim\left(\begin{array}{c}
\ddots \\
P_{2} \\
\ddots \\
-P_{1} \\
\ddots
\end{array}\right)
$$

Similarmente, são provados $I_{C}$ e $I I_{C}$. 
Proposição 3.5. Para quaisquer $A, B \in G L(R)$, vale $A B \sim B A$.

Demonstração. Para um natural $n$ suficientemente grande, podemos supor que ambas as matrizes $A$ e $B$ são matrizes $n \times n$.

A proposição anterior nos garante que

$$
A B \sim\left(\begin{array}{cc}
A B & 0 \\
0 & I_{n}
\end{array}\right) \sim\left(\begin{array}{cc}
A B & A \\
0 & I_{n}
\end{array}\right) \sim\left(\begin{array}{cc}
0 & A \\
-B & I_{n}
\end{array}\right) \sim\left(\begin{array}{cc}
0 & A \\
-B & 0
\end{array}\right) .
$$

Por simetria, temos

$$
B A \sim\left(\begin{array}{cc}
0 & B \\
-A & 0
\end{array}\right)
$$

Logo,

$$
A B \sim\left(\begin{array}{cc}
0 & A \\
-B & 0
\end{array}\right) \sim\left(\begin{array}{cc}
A & 0 \\
0 & B
\end{array}\right) \sim\left(\begin{array}{cc}
0 & B \\
-A & 0
\end{array}\right) \sim B A
$$

Proposição 3.6. $E(R)$ é o subgrupo comutador de $G L(R)$.

Demonstração. Primeiramente, observe que se $E \in E(R)$ e se $X \in G L(R)$, então a proposição anterior nos fornece:

$$
X E X^{-1}=(X E) X^{-1} \sim X^{-1}(X E)=E
$$

Logo, $X E X^{-1} \in E(R)$.

Seja $A B A^{-1} B^{-1}$ um comutador. Pela última proposição temos que existem matrizes $E_{1}, E_{2} \in E(R)$, tais que $A B=E_{1}(B A) E_{2}$. Segue deste fato e da observação inicial, com $X=B A$, que $A B\left(A^{-1} B^{-1}\right)=\left[E_{1}(B A) E_{2}\right](B A)^{-1}=\left(E_{1} X E_{2}\right) X^{-1}=E_{1}\left(X E_{2} X^{-1}\right) \in E(R)$.

Assim, o subgrupo comutador está contido em $E(R)$.

Mostremos agora que a inclusão contrária também é válida e que portanto $E(R)$ é o subgrupo comutador de $G L(R)$. 
Temos que $E(R)$ é gerado pelas matrizes da forma $\left(I_{n}+a E_{i, j}^{n}\right)$, com $i \neq j$ e $a \in R$.

Agora, afirmamos que a matriz $\left(I_{n}+a E_{i, j}^{n}\right)$ é um elemento comutador. De fato,

$$
\left(I_{n}+a E_{i, j}^{n}\right)=\left(I_{n}+a E_{i, k}^{n}\right)\left(I_{n}+E_{k, j}^{n}\right)\left(I_{n}-a E_{i, k}^{n}\right)\left(I_{n}+E_{k, j}^{n}\right) .
$$

Logo, $E(R)$ está contido no subgrupo comutador do grupo $G L(R)$, o que finaliza a demonstração da proposição.

O resultado enunciado a seguir é um resultado básico da álgebra elementar, por isso vamos omitir sua demonstração.

Proposição 3.7. Se $H$ é um subgrupo de $G L(R)$ contendo $E(R)$ então $H$ é normal e $G L(R) / H$ é um grupo abeliano.

Assim, o grupo $G L(R) / E(R)$ é abeliano e a relação de equivalência definida anteriormente coincide com a relação entre duas matrizes da mesma classe de $G L(R) / E(R)$.

Agora, seja $G$ um subgrupo do grupo das unidades de $R$. Denote por $E_{G}$ o subgrupo de $G L(R)$ gerado por $E(R)$ e pelas matrizes da forma:

$$
\left(\begin{array}{ccccccc}
1 & 0 & 0 & \ldots & \ldots & \ldots & 0 \\
0 & 1 & 0 & \ldots & \ldots & \ldots & 0 \\
\vdots & & \ddots & & & & \vdots \\
0 & \ldots & \ldots & g & 0 & \ldots \ldots & 0 \\
0 & \ldots & \ldots & 1 & 0 & \ldots & 0 \\
\vdots & & & & \ddots & & \vdots \\
0 & \ldots & \ldots & \ldots & \ldots & \ldots & 1
\end{array}\right)
$$

onde $g \in G$.

Denotaremos o grupo abeliano $G L(R) / E_{G}$ por $K_{G}(R)$.

Seja $\tau: G L(R) \rightarrow K_{G}(R)$ a aplicação quociente. 
Definição 3.4. Dada uma matriz $A \in G L(R)$, chamamos de torção da matriz $A$ o elemento $\tau(A) \in K_{G}(R)$.

Observe que neste caso duas matrizes de $G L(R)$ terão mesma torção se e somente se uma pode ser transformada na outra por meio de uma seqüência finita de operações dos tipos II, III e V e através de multiplicação de linhas/colunas por um elemento de $G$ à esquerda/à direita.

Por conveniência vamos considerar que o grupo abeliano $K_{G}(R)$ é aditivo, ou seja, denotaremos $\tau(A B)=\tau(A)+\tau(B)$.

Facilmente verifica-se que a relação:

$$
A \sim B \Longleftrightarrow \tau(A)=\tau(B) \in K_{G}(R)
$$

define uma relação de equivalência em $G L(R)$.

Analisemos o grupo $K_{G}(R)$ para as escolhas mais comuns de G:

- Se $G=\{1\}$, então $K_{G}(R)=G L(R) / E(R)$, já que $E_{G}=E(R)$.

- Se $G=\{-1,1\}$, então vamos ter que duas matrizes $A, B \in G L(R)$ terão mesma torção em $K_{G}(R)$ se e somente se $A$ pode ser transformada em $B$ através de uma sequiência finita de operações dos tipos II, III, V e operações que multiplicam uma linha ou coluna por -1 . Assim, dada $A \in G L(R), \tau(A)=\tau(-A)$.

Uma propriedade interessante no caso onde $-1 \in G$ é que, por $I I_{R}$ e $I I_{C}$, podemos trocar linhas e colunas de uma matriz qualquer sem modificar sua torção.

Agora, seja $G$ um grupo qualquer. Considere o anel com unidade $\mathbb{Z} G$. Como vimos na seção anterior, trata-se de um anel que satisfaz $\left(^{*}\right)$.

Definição 3.5. Considere o grupo $K_{T}(\mathbb{Z} G)=G L(\mathbb{Z} G) / E_{T}$, onde $T$ é o conjunto das unidades triviais de $\mathbb{Z} G$ apresentado na seção (2.5). Este grupo será denotado por $W h(G)$ e será chamado de grupo de Whitehead do grupo $G$. 
Observe que, se $A, B \in G L(\mathbb{Z} G)$, então $\tau(A)=\tau(B)$ se e somente se $A$ pode ser transformada em $B$ por uma seqüência finita de operações dos tipos I - V.

Sejam $R$ e $R^{\prime}$ anéis satisfazendo (*) e sejam $G$ e $G^{\prime}$ subgrupos dos grupos das unidades de $R$ e $R^{\prime}$, respectivamente. Seja $f: R \rightarrow R^{\prime}$ um homomorfismo de anéis tal que $f(G) \subset G^{\prime}$. Então obtemos um homomorfismo de grupos $f_{*}: K_{G}(R) \rightarrow K_{G^{\prime}}\left(R^{\prime}\right)$ dado por $f_{*}\left(\tau\left(a_{i j}\right)\right)=\tau\left(f\left(a_{i j}\right)\right)$.

Obtemos então um funtor covariante da categoria dos pares $(R, G)$, descritos acima, e dos homomorfismos de anéis $f: R \rightarrow R^{\prime}$ satisfazendo $f(G) \subset G^{\prime}$ na categoria dos grupos abelianos e homomorfismo de grupos. Este funtor associa a cada par $(R, G)$ o grupo abeliano $K_{G}(R)$ e a cada homomorfismo de anéis $f$ como acima um homomorfismo de grupos $f_{*}$.

Obtemos também um outro funtor da categoria dos grupos e homomorfismos de grupos na categoria dos grupos abelianos e homomorfismos de grupos que associa a cada grupo $G$ o grupo abeliano $W h(G)$ e a cada homomorfismo $f: G \rightarrow G^{\prime}$ um homomorfismo $f_{*}: W h(G) \rightarrow W h\left(G^{\prime}\right)$ que é induzido por $f$ da seguinte maneira: a partir de $f: G \rightarrow G^{\prime}$ induzimos um homomorfismo de anéis $f: \mathbb{Z} G \rightarrow \mathbb{Z} G^{\prime}$, também denotado por $f$, dado por $f\left(\sum_{i} n_{i} g_{i}\right)=\sum_{i} n_{i} f\left(g_{i}\right)$. Note que $f(T) \subset T^{\prime}$, onde $T$ e $T^{\prime}$ são os grupos das unidades triviais de $\mathbb{Z} G$ e $\mathbb{Z} G^{\prime}$, respectivamente. Logo, podemos obter um homomorfismo de anéis $f_{*}: W h(G) \rightarrow W h\left(G^{\prime}\right)$ como na definição do funtor anterior.

Proposição 3.8. Se $g \in G$ e se $f: G \rightarrow G$ é o homomorfismo de grupos dado por $f(x)=g x g^{-1}, \forall x \in G$, então o homomorfismo induzido $f_{*}$ : $W h(G) \rightarrow W h(G)$ é a aplicação identidade.

Demonstração. Seja $\tau: G L(\mathbb{Z} G) \rightarrow W h(G)$ a aplicação quociente. 
Denote o elemento $\tau\left(\left(a_{i j}\right)\right) \in W h(G)$ por

$$
\left[\left(\begin{array}{cccc}
a_{11} & a_{12} & \ldots & a_{1 n} \\
a_{21} & a_{22} & \ldots & a_{2 n} \\
\vdots & \vdots & & \vdots \\
a_{n 1} & a_{n 2} & \ldots & a_{n n}
\end{array}\right)\right]
$$

Temos que $f_{*}$ associa a este elemento de $W h(G)$ o elemento

$$
\begin{aligned}
& {\left[\left(\begin{array}{cccc}
f\left(a_{11}\right) & f\left(a_{12}\right) & \ldots & f\left(a_{1 n}\right) \\
f\left(a_{21}\right) & f\left(a_{22}\right) & \ldots & f\left(a_{2 n}\right) \\
\vdots & \vdots & & \vdots \\
f\left(a_{n 1}\right) & f\left(a_{n 2}\right) & \ldots & f\left(a_{n n}\right)
\end{array}\right)\right]=\left[\left(\begin{array}{cccc}
g a_{11} g^{-1} & g a_{12} g^{-1} & \ldots & g a_{1 n} g^{-1} \\
g a_{21} g^{-1} & g a_{22} g^{-1} & \ldots & g a_{2 n} g^{-1} \\
\vdots & \vdots & & \vdots \\
g a_{n 1} g^{-1} & g a_{n 2} g^{-1} & \ldots & g a_{n n} g^{-1}
\end{array}\right)\right]} \\
& =\left[\left(\begin{array}{ccccc}
g & 0 & 0 & \ldots & 0 \\
0 & g & 0 & \ldots & 0 \\
\vdots & & \ddots & & \vdots \\
0 & \ldots & \ldots & \ldots & g
\end{array}\right)\left(\begin{array}{cccc}
a_{11} & a_{12} & \ldots & a_{1 n} \\
a_{21} & a_{22} & \ldots & a_{2 n} \\
\vdots & \vdots & & \vdots \\
a_{n 1} & a_{n 2} & \ldots & a_{n n}
\end{array}\right)\left(\begin{array}{ccccc}
g^{-1} & 0 & 0 & \ldots & 0 \\
0 & g^{-1} & 0 & \ldots & 0 \\
\vdots & & \ddots & & \vdots \\
0 & \ldots \ldots & \ldots & \ldots & g^{-1}
\end{array}\right)\right] \\
& =\left[\left(\begin{array}{cccc}
a_{11} & a_{12} & \ldots & a_{1 n} \\
a_{21} & a_{22} & \ldots & a_{2 n} \\
\vdots & \vdots & & \vdots \\
a_{n 1} & a_{n 2} & \ldots & a_{n n}
\end{array}\right)\right]
\end{aligned}
$$

pois

$$
\left(\begin{array}{ccccc}
g & 0 & 0 & \ldots & 0 \\
0 & g & 0 & \ldots & 0 \\
\vdots & & \ddots & & \vdots \\
0 & \ldots & \ldots & \ldots & g
\end{array}\right)=\left(\begin{array}{ccccc}
g & 0 & 0 & \ldots & 0 \\
0 & 1 & 0 & \ldots & 0 \\
\vdots & & \ddots & & \vdots \\
0 & \ldots & \ldots & \ldots & 1
\end{array}\right) \ldots\left(\begin{array}{ccccc}
1 & 0 & 0 & \ldots & 0 \\
0 & 1 & 0 & \ldots & 0 \\
\vdots & & \ddots & & \vdots \\
0 & \ldots & \ldots & \ldots & g
\end{array}\right)
$$

e cada fator acima tem torção nula, qualquer que seja $g \in G$.

Proposição 3.9. Sejam $A, B$ e $X$ matrizes $n \times n, m \times m$ e $n \times m$ sobre $R$, respectivamente.

(i) Se A tem inversa à direita ou $B$ tem inversa à esquerda, então a matriz

$$
\left(\begin{array}{cc}
A & X \\
0 & B
\end{array}\right)
$$

é não-singular $\Longleftrightarrow A$ e $B$ são não-singulares. 
(ii) Se $A$ e $B$ são não-singulares, então

$$
\tau\left(\begin{array}{cc}
A & X \\
0 & B
\end{array}\right)=\tau(A)+\tau(B)
$$

onde $G$ é um subgrupo qualquer das unidades de $R$ e $\tau: G L(R) \rightarrow$ $K_{G}(R)$ é a aplicação quociente.

Demonstração. (i) Se $A$ tem inverso à direita, então temos

$$
\left(\begin{array}{cc}
A & 0 \\
0 & B
\end{array}\right)=\left(\begin{array}{cc}
A & X \\
0 & B
\end{array}\right)\left(\begin{array}{cc}
I_{n} & -A^{-1} X \\
0 & I_{m}
\end{array}\right)
$$

Se, por sua vez, $B$ tem inverso à esquerda, então

$$
\left(\begin{array}{cc}
A & 0 \\
0 & B
\end{array}\right)=\left(\begin{array}{cc}
I_{n} & -X B^{-1} \\
0 & I_{m}
\end{array}\right)\left(\begin{array}{cc}
A & X \\
0 & B
\end{array}\right)
$$

Observe que ambas as matrizes $\left(\begin{array}{cc}I_{n} & -A^{-1} X \\ 0 & I_{m}\end{array}\right)$ e $\left(\begin{array}{cc}I_{n} & -X B^{-1} \\ 0 & I_{m}\end{array}\right)$ são não-singulares pois resultam de operações elementares realizadas na matriz identidade $I_{n+m}$.

Segue que $\left(\begin{array}{cc}A & X \\ 0 & B\end{array}\right)$ é não-singular se e somente se $\left(\begin{array}{cc}A & 0 \\ 0 & B\end{array}\right)$ é nãosingular, mas isto ocorre se e somente se $A$ e $B$ são não-singulares.

(ii) Se $A$ e $B$ são não-singulares, então pelo item anterior temos que

$$
\begin{aligned}
& \tau\left(\begin{array}{cc}
A & 0 \\
0 & B
\end{array}\right)=\tau\left(\left(\begin{array}{cc}
A & X \\
0 & B
\end{array}\right)\left(\begin{array}{cc}
I_{n} & -A^{-1} X \\
0 & I_{m}
\end{array}\right)\right) \\
= & \tau\left(\begin{array}{cc}
A & X \\
0 & B
\end{array}\right)+\tau\left(\begin{array}{cc}
I_{n} & -A^{-1} X \\
0 & I_{m}
\end{array}\right)=\tau\left(\begin{array}{cc}
A & X \\
0 & B
\end{array}\right)
\end{aligned}
$$

Assim,

$$
\begin{aligned}
\tau\left(\begin{array}{cc}
A & X \\
0 & B
\end{array}\right) & =\tau\left(\begin{array}{cc}
A & 0 \\
0 & B
\end{array}\right)=\tau\left(\left(\begin{array}{cc}
A & 0 \\
0 & I_{m}
\end{array}\right)\left(\begin{array}{cc}
I_{n} & 0 \\
0 & B
\end{array}\right)\right) \\
& =\tau\left(\begin{array}{cc}
A & 0 \\
0 & I_{m}
\end{array}\right)+\tau\left(\begin{array}{cc}
I_{n} & 0 \\
0 & B
\end{array}\right)
\end{aligned}
$$


Mas, veja que,

$$
\left(\begin{array}{cc}
I_{n} & 0 \\
0 & B
\end{array}\right) \stackrel{I I_{R}}{\sim}\left(\begin{array}{cc}
0 & B \\
(-1)^{m} I_{n} & 0
\end{array}\right) \stackrel{I I_{C}}{\sim}\left(\begin{array}{cc}
B & 0 \\
0 & (-1)^{2 m} I_{n}
\end{array}\right)
$$

Então,

$$
\begin{aligned}
& \tau\left(\begin{array}{cc}
A & X \\
0 & B
\end{array}\right)=\tau\left(\begin{array}{cc}
A & 0 \\
0 & I_{m}
\end{array}\right)+\tau\left(\begin{array}{cc}
I_{n} & 0 \\
0 & B
\end{array}\right) \\
= & \tau\left(\begin{array}{cc}
A & 0 \\
0 & I_{m}
\end{array}\right)+\tau\left(\begin{array}{cc}
B & 0 \\
0 & I_{n}
\end{array}\right)=\tau(A)+\tau(B)
\end{aligned}
$$

No caso de $R$ ser um anel comutativo a teoria usual de determinantes é aplicável e pode ser útil para lidar com torções, já que as operações elementares que transformam uma matriz numa outra matriz de mesma torção podem no máximo alterar o determinante multiplicando-o por um elemento de $G$.

Teorema 3.10. Sejam $R$ um anel comutativo, $G$ um subgrupo do grupo $U$ das unidades de $R$ e $\tau_{G}: G L(R) \rightarrow K_{G}(R)$ a aplicação quociente. Considere o subgrupo $S L(R)$ de $G L(R)$ constituído das matrizes com determinante 1. Seja $S K_{1}(R)=\tau_{G}(S L(R))$. Afirmamos que $S K_{1}(R)$ independe da escolha do subgrupo $G$ de $U$.

Demonstração. Considere a aplicação $\pi: K_{1}(R) \rightarrow K_{G}(R)$ que associa a cada elemento $\tau_{1}(A) \in K_{1}(R)$ o elemento $\tau_{G}(A)$. É trivial verificar que esta aplicação é um homomorfismo de grupos bem definido.

Agora, considere a restrição $\pi \mid \tau_{1}(S L(R)): \tau_{1}(S L(R)) \rightarrow \tau_{G}(S L(R))$. Mostremos que este epimorfismo é na realidade um isomorfismo

Para isso, basta mostrar que se $A \in E_{G} \operatorname{com} \operatorname{det}(A)=1$, então $A \in E(R)$.

Assim, tome $A \in E_{G} \operatorname{com} \operatorname{det}(A)=1$. Então podemos supor que $A=$ $E_{1} E_{2} \ldots E_{n}$, tal que cada $E_{i}$ ou é uma matriz elementar $\left(I_{n}+a_{i} E_{j, k}\right), j \neq$ 
$k$ e $a_{i} \in R$ ou é da forma

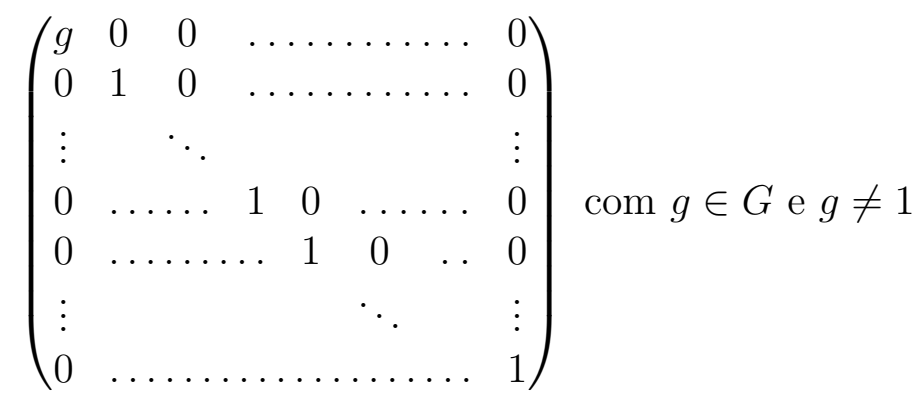

- que chamaremos de matriz do tipo II.

Podemos fazer esta suposição pois, pelas propriedades $I I_{R}$ e $I I_{C}$, temos que

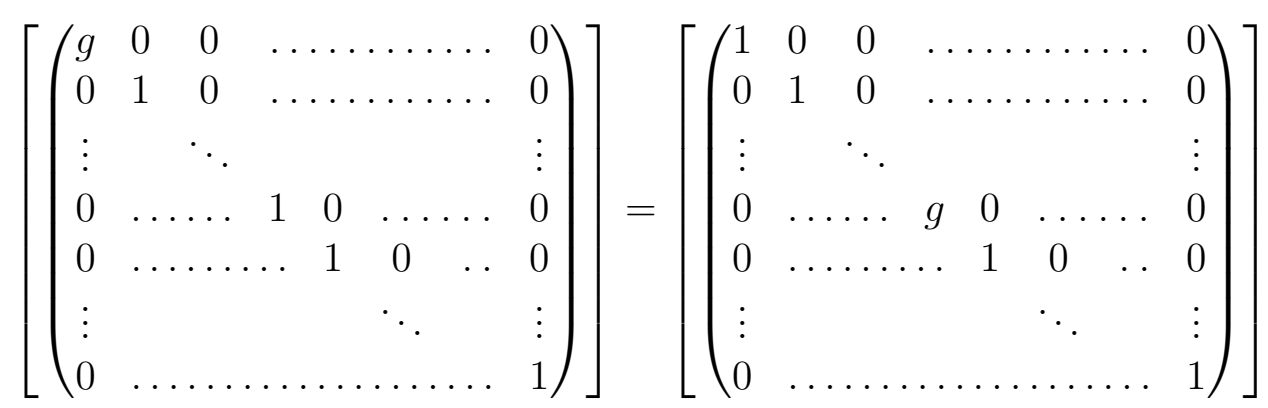

$\in G L(R) / E(R)=K_{1}(R)$, onde os colchetes indicam a torção.

Podemos ainda supor que se $E_{i}$ é do tipo II, então $E_{i+1}$ é do tipo I, pois caso contrário $E=E_{i} E_{i+1}$ ou é a matriz identidade ou é do tipo II e então poderíamos ignorar $E$ no primeiro caso ou substituir $E_{i} E_{i+1}$ na fatoração de $A$ por $E$ no segundo caso.

Seja $m$ o número de fatores $E_{i}$ que são do tipo II que aparecem numa fatoração de $A$ que satisfaça ambas as suposições acima.

Veja que $m>1$, pois se $m=1$, então $\operatorname{det}(A)=g$, para algum $g \in G, g \neq$ 1, o que contradiz nossa hipótese.

Se $m=2$, então sejam $E_{i}$ e $E_{j}$, com $i<j$, os únicos dois fatores da forma 
II. Então, obrigatoriamente temos que, se

$$
E_{i}=\left(\begin{array}{ccccc}
g & 0 & 0 & \ldots & 0 \\
0 & 1 & 0 & \ldots & 0 \\
\vdots & & \ddots & & \vdots \\
0 & \ldots & \ldots & 1
\end{array}\right)
$$

então

$$
E_{j}=\left(\begin{array}{ccccc}
g^{-1} & 0 & 0 & \ldots & 0 \\
0 & 1 & 0 & \ldots & 0 \\
\vdots & & \ddots & & \vdots \\
0 & \ldots & \ldots & 1
\end{array}\right)
$$

pois $1=\operatorname{det}(A)=\operatorname{det}\left(E_{i}\right) \operatorname{det}\left(E_{j}\right)=g \operatorname{det}\left(E_{j}\right) \Rightarrow \operatorname{det}\left(E_{j}\right)=g^{-1}$, mostrando que a afirmação anterior é verdadeira.

Então, temos

$$
\begin{gathered}
A=E_{1} \ldots E_{i} E_{i+1} \ldots E_{j-1} E_{j} \ldots E_{n} \\
=E_{1} \ldots\left(E_{i} E_{j}\right)\left(E_{i} E_{i+1} E_{j}\right)\left(E_{i} \ldots E_{j}\right)\left(E_{i} E_{j-1} E_{j}\right) \ldots E_{n}
\end{gathered}
$$

pois $E_{j} E_{i}$ é a matriz identidade.

Agora, veja que, como cada $E_{k}, k \neq i, j$ é uma matriz elementar, $E_{i} E_{k} E_{j}$ também é uma matriz elementar. E então, claramente $A \in E(R)$.

Para $m>2$ procedemos da seguinte forma: sejam $1 \leq i_{1}<i_{2}<\cdots<$ $i_{m} \leq n$ índices tais que cada $E_{i_{j}}$ tem a forma

$$
\left(\begin{array}{ccccc}
g_{j} & 0 & 0 & \ldots & 0 \\
0 & 1 & 0 & \ldots & 0 \\
\vdots & & \ddots & & \vdots \\
0 & \ldots & \ldots & \ldots & 1
\end{array}\right)
$$

com $g_{j} \in G$ e $g_{j} \neq 1$. Para facilitar vamos escrever $i=i_{1}$.

Então

$$
\begin{gathered}
A=E_{1} \ldots E_{i-1} E_{i} E_{i+1} E_{i+2} \ldots E_{i_{2}} E_{i_{2}+1} \ldots E_{n} \\
=E_{1} \ldots E_{i-1}\left(E_{i} E_{i+1} E_{i}^{-1}\right)\left(E_{i} E_{i+2} E_{i}^{-1}\right)\left(E_{i} \ldots E_{i}^{-1}\right)\left(E_{i} E_{i_{2}}\right) E_{i_{2}+1} \ldots E_{n} .
\end{gathered}
$$


Mas, observe que, para $k \in \mathbb{Z}$, com $i<k<i_{2}, F_{k}=E_{i}^{-1} E_{k} E_{i}$ é uma matriz elementar e $F=E_{i} E_{i_{2}}$ tem a forma II.

Assim, $A=E_{1} \ldots E_{i-1} F_{i+1} F_{i+2} \ldots F_{i_{2}-1} F E_{i_{2}+1} \ldots E_{n}$ é uma fatoração de $A$ (satisfazendo as suposições iniciais) que tem $m-1$ elementos da forma II. Repetindo este processo mais algumas vezes, encontraremos uma fatoração de $A$ que tem apenas duas matrizes da forma II e neste caso já mostramos que $A \in E(R)$.

Teorema 3.11. Nas condições do teorema acima, tem-se que a seqüência:

$$
0 \rightarrow S K_{1}(R) \stackrel{\subset}{\longrightarrow} K_{G}(R) \stackrel{[\mathrm{det}]}{\underset{s}{\rightleftarrows}} U / G \rightarrow 0
$$

com $[\operatorname{det}](\tau(A))=(\operatorname{det}(A)) G$ e $s(u G)=\tau((u))$, onde $(u)$ indica a matriz $1 \times 1$ que tem como entrada o elemento $u \in U$, é uma seqüência exata curta que cinde. No caso de $R$ ser um corpo, a aplicação [det] é um isomorfismo.

Demonstração. Tome $A \in G L(R) \operatorname{com} \operatorname{det}(A)=g \in G$. Seja $B=\left(g^{-1}\right) A$. Então $\tau_{G}(A)=\tau_{G}(B) \in K_{G}(R) \operatorname{e} \operatorname{det}(B)=1$. Logo, $\tau_{G}(B) \in S K_{1}(R)$, donde $\operatorname{Ker}[\operatorname{det}] \subset S K_{1}(R)$. A inclusão de $S K_{1}(R)$ em $\operatorname{Ker}[\operatorname{det}]$ segue trivialmente. Assim, a exatidão em $K_{G}(R)$ está provada.

A exatidão em $S K_{1}(R)$ é evidente.

Agora, observe que [det] $s=1_{U / G}$, o que prova a exatidão em $U / G$ e também que a seqüência dada cinde.

No caso de $R$ ser um corpo, temos que $S K_{1}(R)=0$, pois tomando $G^{\prime}=$ $U=R-0$ temos que $K_{G^{\prime}}(R)=0$ e então o teorema anterior nos garante a validade da afirmação. Segue deste fato e da exatidão da seqüência dada que [det] é um isomorfismo.

Terminamos esta seção com uma ressalva: é possível que uma matriz $n \times n$ não possa ser transformada em uma outra matriz $n \times n$ através de operações 
elementares mas que, no entanto, quando consideradas como matrizes de $G L(R)$ ambas tenham a mesma torção. O exemplo a seguir ilustra esta situação.

Exemplo 1. Seja $G$ um grupo não-comutativo gerado por dois elementos, $x$ e $y$, com $y^{2}=1$. Considere os elementos $a=1-y$ e $b=x(1+y)$ de $\mathbb{Z} G$. Veja que $a b=x+x y-y x-y x y \neq 0$ e $b a=0$. A matriz $1 \times 1,(1-a b)$ não é uma matriz elementar e $1-a b$ não é uma unidade trivial, mas como esta matriz representa o mesmo elemento em $W h(G)$ que a matriz

$$
\left(\begin{array}{cc}
1-a b & 0 \\
0 & 1
\end{array}\right)=\left(\begin{array}{ll}
1 & 0 \\
b & 1
\end{array}\right)\left(\begin{array}{ll}
1 & a \\
0 & 1
\end{array}\right)\left(\begin{array}{ll}
1 & 0 \\
b & 1
\end{array}\right)^{-1}\left(\begin{array}{ll}
1 & a \\
0 & 1
\end{array}\right)^{-1}
$$

sua torção é nula.

\section{$3.3(R, G)$-complexos}

De agora em diante $G$ sempre denotará um subgrupo do grupo das unidades do anel $R$ contendo o elemento -1 .

Definição 3.6. Chamamos de $(R, G)$-módulo um $R$-módulo livre $M$ junto com uma família de bases $B$ que satisfaz a seguinte condição:

Se $b$ e $b^{\prime}$ são bases para $M$ e se $b \in B$, então $b^{\prime} \in B \Longleftrightarrow \tau\left(\left\langle b / b^{\prime}\right\rangle\right)=0 \in K_{G}(R)$.

A família $B$ é chamada de família distinguida de bases para $M$ e cada base $b \in B$ é chamada de base distinguida de $M$.

Definição 3.7. Sejam $M_{1}$ e $M_{2}(R, G)$-módulos, $f: M_{1} \rightarrow M_{2}$ um isomorfismo de módulos e $A$ a matriz de $f$ com relação a um par qualquer de bases distinguidas para $M_{1}$ e $M_{2}$. Então definimos a torção de $f$ e indicamos por $\tau(f)$ o elemento $\tau(A) \in K_{G}(R)$.

No caso de $\tau(f)=0 \in K_{G}(R)$, dizemos que $f$ é um isomorfismo simples de $(R, G)$-módulos e indicamos esta situação por $f: M_{1} \cong_{S} M_{2}$. 
Observe que se $x$ e $x^{\prime}$ são bases distinguidas para $M_{1}, y$ e $y^{\prime}$ são bases distinguidas para $M_{2}, A$ é a matriz de $f$ com relação às bases $x$ e $y$ e $B$ é a matriz de $f$ com relação às bases $x^{\prime}$ e $y^{\prime}$, então

$$
\begin{aligned}
\tau(A) & =\tau\left(\langle f\rangle_{x, y}\right) \\
& =\tau\left(\left\langle x / x^{\prime}\right\rangle\langle f\rangle_{x^{\prime}, y^{\prime}}\left\langle y^{\prime} \mid y\right\rangle\right) \\
& =\tau\left(\left\langle x / x^{\prime}\right\rangle\right)+\tau\left(\langle f\rangle_{x^{\prime}, y^{\prime}}\right)+\tau\left(\left\langle y^{\prime} / y\right\rangle\right) \\
& =\tau\left(\langle f\rangle_{x^{\prime}, y^{\prime}}\right)=\tau(B),
\end{aligned}
$$

o que garante a coerência da definição acima.

Definição 3.8. Seja $C$ um complexo de cadeia

$$
C: 0 \rightarrow C_{n} \stackrel{d_{n}}{\longrightarrow} C_{n-1} \rightarrow \cdots \rightarrow C_{0} \rightarrow 0,
$$

tal que cada $C_{i}$ é um $(R, G)$-módulo e cada operador $d_{i}$ é um homomorfismo de módulos. Neste caso dizemos que $C$ é um $(R, G)$-complexo.

Uma base distinguida para $C$ significa uma base $c=\bigcup_{i} c_{i}$, onde cada $c_{i}$ é uma base distinguida para $C_{i}$.

Definição 3.9. Seja $G$ um grupo e $C$ um $(\mathbb{Z} G, T)$-complexo, onde $T$ denota o grupo das unidades triviais de $\mathbb{Z} G$. Um complexo como este é chamado de $W h(G)$-complexo.

Definição 3.10. Dizemos que uma aplicação de cadeia entre $(R, G)$ complexos $f: C \rightarrow C^{\prime}$ é um isomorfismo simples se $f_{i}: C_{i} \cong_{S} C_{i}^{\prime}, \forall i$. Neste caso, escrevemos $f: C \cong{ }_{S} C^{\prime}$.

Proposição 3.12. Seja $f: C \rightarrow C^{\prime}$ uma aplicação entre $(R, G)$-complexos. Então $f: C \cong{ }_{S} C^{\prime}$ se e somente se existem bases distinguidas com respeito das quais, para cada $i$, a matriz de $f_{i}$ é a matriz identidade e as matrizes de $d_{i}: C_{i} \rightarrow C_{i-1}$ e $d_{i}^{\prime}: C_{i}^{\prime} \rightarrow C_{i-1}^{\prime}$ são iguais, onde $d$ e $d^{\prime}$ são os operadores bordo dos complexos $C$ e $C^{\prime}$, respectivamente.

Demonstração. $(\Rightarrow)$ Seja $i$ um índice arbitrário. Tome bases distinguidas $b_{i}=\left\{x_{1}, \ldots, x_{p}\right\}$ e $b_{i}^{\prime}=\left\{y_{1}, \ldots, y_{p}\right\}$ para $C_{i}$ e $C_{i}^{\prime}$, respectivamente. Denote $A=\left\langle f_{i}\right\rangle_{b_{i}, b_{i}^{\prime}}$. 
Como $f_{i}$ é um isomorfismo de módulos, $\left\{f_{i}\left(x_{1}\right), \ldots, f_{i}\left(x_{p}\right)\right\}$ forma uma base para o $R$-módulo $C_{i}^{\prime}$. Denote esta base por $c_{i}^{\prime}$.

Observe que $c_{i}^{\prime}$ também é uma base distinguida para $C_{i}^{\prime}$, pois $\left\langle c_{i}^{\prime} / b_{i}^{\prime}\right\rangle=A$ e então $\tau\left(\left\langle c_{i}^{\prime} / b_{i}^{\prime}\right\rangle\right)=\tau(A)=0$, por hipótese.

Com esta base distinguida, temos que $\left\langle f_{i}\right\rangle_{b_{i}, c_{i}^{\prime}}=I_{p}$.

Como $i$ foi tomado arbitrariamente, temos que a existência de bases distinguidas para $C_{i}$ e $C_{i}^{\prime}$ de maneira que a matriz de $f_{i}$ com relação a estas bases é a matriz identidade é válida para todo $i$.

Agora, como $f$ é uma aplicação de cadeia, temos que, para cada $i$, $f_{i-1} d_{i}=d_{i}^{\prime} f_{i}$. Assim, as matrizes dos homomorfismos de módulos $f_{i-1} d_{i} \mathrm{e}$ $d_{i}^{\prime} f_{i}$ coincidem. Em particular, se tomarmos como bases para os módulos $C_{i}$ e $C_{i}^{\prime}$ bases distinguidas como acima, temos

$$
\left\langle f_{i-1} d_{i}\right\rangle=\left\langle d_{i}^{\prime} f_{i}\right\rangle \Rightarrow\left\langle f_{i-1}\right\rangle\left\langle d_{i}\right\rangle=\left\langle d_{i}^{\prime}\right\rangle\left\langle f_{i}\right\rangle \Rightarrow\left\langle d_{i}\right\rangle=\left\langle d_{i}^{\prime}\right\rangle .
$$

$(\Leftarrow)$ Trivial.

\subsection{Complexos de cadeia acíclicos}

Definição 3.11. Dizemos que um $R$-módulo $M$ é estavelmente livre se existe um $R$-módulo livre $F$ tal que $M \oplus F$ é livre.

Proposição 3.13. Seja $M$ um R-módulo estavelmente livre e seja $j: A \rightarrow$ $M$ um epimorfismo de R-módulos. Então existe um homomorfismo de $R$ módulos $s: M \rightarrow A$ tal que $j s=1_{M}$. Este homomorfismo é chamado de seção.

Demonstração. Seja $F$ o $R$-módulo livre tal que $M \oplus F$ é livre.

Como $j: A \rightarrow M$ é um epimorfismo, a aplicação $j \oplus 1_{F}: A \oplus F \rightarrow M \oplus F$ é também um epimorfismo. Claramente, existe uma aplicação $S: M \oplus F \rightarrow$ $A \oplus F$, tal que $\left(j \oplus 1_{F}\right) S=1_{M \oplus F}$. Basta tomar uma base $x=\left\{x_{1}, \ldots, x_{p}\right\}$ 
para $M \oplus F$ e definir $S\left(x_{i}\right)$ como sendo um elemento $y_{i}$ de $A \oplus F$ tal que $\left(j \oplus 1_{F}\right)\left(y_{i}\right)=x_{i}$. Então $s=\pi_{1} S i_{i}$, onde $i_{1}: M \rightarrow M \oplus F$ é o homomorfismo de módulos dado por $i_{1}(m)=m+0, m \in M$, e $\pi_{1}: A \oplus F \rightarrow A$ é a projeção na primeira coordenada, é tal que $j s=1_{M}$. Com efeito, $j s=j \pi_{1} S i_{1}=$ $p_{1}\left(j \oplus 1_{F}\right) S i_{1}$, onde $p_{1}: M \oplus F \rightarrow M$ é também a projeção na primeira coordenada, donde $j s=p_{1} i_{1}=1_{M}$.

Definição 3.12. Chamamos de complexo de cadeia sobre $R$, um complexo de cadeia $C$ com operador bordo $d$, tal que cada $C_{i}$ é um $R$-módulo e cada $d_{i}$ é um homomorfismo de módulos. No caso em que todo $C_{i}$ é livre, então $C$ é chamado de complexo de cadeia livre sobre $R$.

Definição 3.13. Dizemos que um complexo de cadeia $C$ com operador bordo $d$ é acíclico quando, para cada $i, \operatorname{Im}\left(d_{i}\right)=\operatorname{Ker}\left(d_{i-1}\right)$.

Proposição 3.14. Seja $C$ um complexo de cadeia acíclico livre sobre $R$ com operador bordo $d$. Denote $B_{i}=d C_{i+1}, \forall i$. Então valem:

(i) $B_{i}$ é estavelmente livre para todo $i$.

(ii) Para cada $i$, existe um homomorfismo de R-módulos, $\delta_{i}: C_{i} \rightarrow C_{i+1}$, tal que $\delta_{i-1} d_{i}+d_{i+1} \delta_{i}=1_{C_{i}}$. O homomorfismo de grau um $\delta: C \rightarrow C$ é chamado de contração de cadeia.

(iii) Se $\delta: C \rightarrow C$ é uma contração de cadeia qualquer então, para cada $i$, $d \delta \mid B_{i-1}=1$ e $C_{i}=B_{i} \oplus \delta B_{i-1}$.

Demonstração. (i) Para demonstrar este item vamos proceder por indução sobre o índice $i$ de $B_{i}$.

De imediato temos que $B_{0}=d_{1} C_{1}=C_{0}$ que por ser módulo livre, é estavelmente livre.

Suponha que $B_{i-1}$ é estavelmente livre. 
Considere o homomorfismo sobrejetivo $d_{i}: C_{i} \rightarrow B_{i-1}$. A proposição anterior nos garante a existência de um homomorfismo de $R$-módulos $s: B_{i-1} \rightarrow C_{i}$ tal que $d_{i} s=1$.

Considere agora a seqüência

$$
0 \rightarrow B_{i} \stackrel{\subset}{\longrightarrow} C_{i} \stackrel{d_{i}}{\gtrless} B_{i-1} \rightarrow 0
$$

Claramente esta seqüência é exata e cinde.

Segue que $C_{i}=B_{i} \oplus s\left(B_{i-1}\right)$.

Agora, veja que $s$ é uma aplicação injetiva. De fato, sejam $x$ e $y$ elementos de $B_{i-1}$ e suponha que $s(x)=s(y)$, então temos que $d_{i}(s(x))=d_{i}(s(y))$, donde $x=y$. Logo, $s: B_{i-1} \rightarrow s\left(B_{i-1}\right)$ é um isomorfismo de $R$-módulos e como $B_{i-1}$ é estavelmente livre, $s\left(B_{i-1}\right)$ é também estavelmente livre.

Seja $F$ um $R$-módulo livre tal que $s\left(B_{i-1}\right) \oplus F$ é livre. Então temos que $C_{i} \oplus F=B_{i} \oplus\left(s\left(B_{i-1}\right) \oplus F\right)$, donde $B_{i}$ é estavelmente livre.

(ii) Como provamos no item anterior $B_{i-1}$ é estavelmente livre para todo $i$. Segue disto e do fato de $d_{i}: C_{i} \rightarrow B_{i-1}$ ser um homomorfismo sobrejetivo que existe uma seção $\delta_{i}: B_{i-1} \rightarrow C_{i}, \forall i$.

Como na demonstração do item anterior, a seqüência

$$
0 \rightarrow B_{i} \stackrel{\subset}{\longrightarrow} C_{i} \underset{\delta_{i}}{\stackrel{d_{i}}{\gtrless}} B_{i-1} \rightarrow 0
$$

é exata e cinde, donde $C_{i}=B_{i} \oplus \delta_{i}\left(B_{i-1}\right)$.

Defina $\delta: C_{i} \rightarrow C_{i+1}$ da seguinte maneira:

$$
\begin{array}{rr}
C_{i}= & B_{i} \oplus \delta_{i}\left(B_{i-1}\right) \\
& \delta_{i+1} \mid 0 \\
C_{i+1}= & B_{i+1} \oplus \delta_{i+1}\left(B_{i}\right)
\end{array}
$$


Então,

$$
\begin{gathered}
C_{i}=B_{i} \oplus \delta_{i}\left(B_{i-1}\right) \\
C_{i+1}=B_{i+1} \oplus \delta_{i+1}\left(B_{i}\right) \\
C_{i}=B_{i} \oplus \delta_{i}\left(B_{i-1}\right)
\end{gathered}
$$

donde podemos concluir que $d \delta: C_{i}=B_{i} \oplus \delta B_{i-1} \rightarrow C_{i}=B_{i} \oplus \delta B_{i-1}$ é a aplicação $1 \oplus 0$.

Por outro lado,

$$
\begin{gathered}
C_{i}=B_{i} \oplus \delta_{i}\left(B_{i-1}\right) \\
C_{i-1}=B_{i-1} \oplus \delta_{i-1}\left(B_{i-2}\right) \\
\left.\delta_{i}\right|_{i} \\
C_{i}=B_{i} \oplus \delta_{i}\left(B_{i-1}\right)
\end{gathered}
$$

e então concluímos que $\delta d=0 \oplus 1$.

Segue que $d \delta+\delta d=(1 \oplus 0)+(0 \oplus 1)=1_{C_{i}}$.

(iii) Se $\delta$ é uma contração de cadeia de $C$ então $d \delta+\delta d=1$. Logo, $(d \delta+\delta d) \mid$ $B_{i-1}=1_{B_{i-1}}$, mas $(d \delta+\delta d)\left|B_{i-1}=d \delta\right| B_{i-1}+\delta d\left|B_{i-1}=d \delta\right| B_{i-1}$, pois $\delta d \mid B_{i-1}$ é o homomorfismo nulo, já que $d^{2}=0$ e $B_{i-1}=d_{i}\left(C_{i}\right)$.

Considere agora a seqüência

$$
0 \longrightarrow B_{i} \stackrel{\subset}{\longrightarrow} C_{i} \underset{\delta \mid B_{i-1}}{\stackrel{d_{i}}{\rightleftarrows}} B_{i-1} \longrightarrow 0
$$

Claramente é uma seqüência exata que cinde, donde $C_{i}=B_{i} \oplus \delta B_{i-1}$.

Observação 1. O homomorfismo $\delta$ construído na demonstração do item (ii) satisfaz $\delta^{2}=0$, porém nem toda contração de cadeia possui esta propriedade. No entanto, dada uma contração de cadeia qualquer $\delta$, obtemos a partir dela uma outra contração de cadeia $\delta^{\prime}=\delta d \delta$ que satisfaz esta propriedade. 
Proposição 3.15. Seja

$$
0 \rightarrow C^{\prime} \stackrel{i}{\rightarrow} C \stackrel{j}{\rightarrow} C^{\prime \prime} \rightarrow 0
$$

uma seqüência exata de complexos de cadeia sobre $R$, com $C^{\prime \prime}$ livre e acíclico. Então existe uma seção $s: C^{\prime \prime} \rightarrow C$ que é uma aplicação de cadeia e é tal que $(i \oplus s): C^{\prime} \oplus C^{\prime \prime} \rightarrow C$ é um isomorfismo de cadeia.

Demonstração. Como o complexo de cadeia $C^{\prime \prime}$ é acíclico e livre sobre $R$, existe uma contração de cadeia $\delta^{\prime \prime}: C^{\prime \prime} \rightarrow C^{\prime \prime}$.

Para cada $k$, temos que o $R$-módulo $C_{k}^{\prime \prime}$ é livre e que, pela exatidão da seqüência de complexos de cadeia do enunciado, $j_{k}: C_{k} \rightarrow C_{k}^{\prime \prime}$ é um homomorfismo sobrejetivo. Segue, pelo resultado (3.13), que existe uma seção $\sigma_{k}: C_{k}^{\prime \prime} \rightarrow C_{k}$.

Observe que não temos garantia nenhuma que $\sigma$ é uma aplicação de cadeia.

Defina $s=d \sigma \delta^{\prime \prime}+\sigma \delta^{\prime \prime} d^{\prime \prime}: C_{k}^{\prime \prime} \rightarrow C_{k}$. Veja que $d s-s d^{\prime \prime}=d \sigma \delta^{\prime \prime} d^{\prime \prime}-$ $d \sigma \delta^{\prime \prime} d^{\prime \prime}=0$, donde $d s=s d$. Veja também que $j s=j d \sigma \delta^{\prime \prime}+j \sigma \delta^{\prime \prime} d^{\prime \prime}=$ $j d \sigma \delta^{\prime \prime}+\delta^{\prime \prime} d^{\prime \prime}=d^{\prime \prime} j \sigma \delta^{\prime \prime}+\delta^{\prime \prime} d^{\prime \prime}=d^{\prime \prime} \delta^{\prime \prime}+\delta^{\prime \prime} d^{\prime \prime}=1$. Assim, $s$ é uma seção para j que também é uma aplicação de cadeia.

Agora, temos que $C_{k}=i\left(C_{k}^{\prime}\right) \oplus s\left(C_{k}^{\prime \prime}\right)$, segue que o homomorfismo $(i \oplus s)$ : $C_{k}^{\prime} \oplus C_{k}^{\prime \prime} \rightarrow C_{k}$ é sobrejetivo. Além disso, temos que $i$ e $s$ são aplicações injetivas, $i$ pela exatidão da seqüência de complexos de cadeia apresentada no enunciado e $s$ por ser uma seção. Logo, $(i \oplus s)$ é também injetiva.

Por fim, veja que $d(i \oplus s)-(i \oplus s)\left(d^{\prime} \oplus d^{\prime \prime}\right)=(d i \oplus d s)-\left(i d^{\prime} \oplus s d^{\prime \prime}\right)=$ $(d i \oplus d s)-(d i \oplus d s)=0$, donde $(i \oplus s)$ é uma aplicação de cadeia.

Assim, $(i \oplus s): C^{\prime} \oplus C^{\prime \prime} \rightarrow C$ é um isomorfismo de cadeia.

Lema 3.16. Seja $C$ um $(R, G)$-complexo de cadeia acíclico com contrações de cadeia $\delta$ e $\bar{\delta}$. Fixado $i$, considere a seguinte aplicação:

$$
1 \oplus \delta d: C_{i}=B_{i} \oplus \bar{\delta} B_{i-1} \longrightarrow C_{i}=B_{i} \oplus \delta B_{i-1} .
$$


Trata-se de um isomorfismo simples. Além disso, se $B_{i}$ e $B_{i-1}$ são $R$-módulos livres com bases $b_{i}$ e $b_{i-1}$, respectivamente, e se $c_{i}$ é uma base para $C_{i}$, então

$$
\tau\left\langle b_{i} \cup \delta b_{i-1} / c_{i}\right\rangle=\tau\left\langle b_{i} \cup \bar{\delta} b_{i-1} / c_{i}\right\rangle .
$$

Demonstração. Inicialmente, observe que $\delta d\left(\bar{\delta} B_{i-1}\right)=\delta B_{i-1}$, pois como vimos no resultado $3.14 d \bar{\delta} \mid B_{i-1}=1$. Segue que a aplicação $\delta d \mid\left(\bar{\delta} B_{i-1}\right)$ : $\bar{\delta} B_{i-1} \rightarrow \delta B_{i-1}$ é sobrejetiva. Por simplicidade, vamos denotar a restrição $\delta d \mid\left(\bar{\delta} B_{i-1}\right)$ por $\delta d$.

Este homomorfismo é também injetivo, pois se $x \in B_{i-1}$ é tal que $\delta d(\bar{\delta}(x))=0$, então $\delta(x)=0$. Mas, como $\delta \mid B_{i-1}$ é injetiva, uma vez que $d \delta \mid B_{i-1}=1, x=0$, donde $\bar{\delta}(x)=0$.

Assim, $\delta d: \bar{\delta} B_{i-1} \rightarrow \delta B_{i-1}$ é um isomorfismo.

Segue que $1 \oplus \delta d: C_{i}=B_{i} \oplus \bar{\delta} B_{i-1} \rightarrow C_{i}=B_{i} \oplus \delta B_{i-1}$ é um isomorfismo. Denotaremos esta aplicação por $g$.

Temos que $\bar{\delta} \mid B_{i-1}: B_{i-1} \rightarrow \bar{\delta} B_{i-1}$ é um isomorfismo. Evidentemente, esta aplicação é um epimorfismo. Sua injetividade é garantida pelo fato de $d \bar{\delta} \mid B_{i-1}=1$ que é satisfeito por toda contração de cadeia.

Assim, temos que para todo $i, B_{i}$ e $\bar{\delta} B_{i-1}$ são estavelmente livres. Segue que existem $R$-módulos livres $F_{1}$ e $F_{2}$ tais que $F_{1} \oplus B_{i}$ e $\bar{\delta} B_{i-1} \oplus F_{2}$ são $R$ módulos livres.

Agora, seja $c$ uma base para o $R$-módulo $F_{1} \oplus C_{i} \oplus F_{2}$ constituída da união de bases, uma para $F_{1}$, uma para $C_{i}$ e uma para $F_{2}$. Defina $G=1_{F_{1}} \oplus g \oplus 1_{F_{2}}$ : $F_{1} \oplus C_{i} \oplus F_{2} \rightarrow F_{1} \oplus C_{i} \oplus F_{2}$.

Temos que

$$
\langle G\rangle_{c, c}=\left(\begin{array}{ccc}
I & 0 & 0 \\
0 & \langle g\rangle & 0 \\
0 & 0 & I
\end{array}\right)
$$

Como $G$ é um isomorfismo de $R$-módulos, a matriz $\langle G\rangle_{c, c}$ é invertível, logo podemos determinar sua torção em $K_{G}(R)$. Pelo mesmo motivo, podemos determinar a torção de $\langle g\rangle$ em $K_{G}(R)$. 
Observe que, pela proposição 3.9$), \tau\left(\langle G\rangle_{c, c}\right)=\tau(\langle g\rangle)$.

Agora, sejam $b_{1}$ uma base para $F_{1} \oplus B_{i}$ e $b_{2}$ uma base para $\bar{\delta} B_{i-1} \oplus F_{2}$. Então $b=b_{1} \cup b_{2}$ é uma base para $F_{1} \oplus C_{i} \oplus F_{2}$.

Antes de continuar, veja que se $A, B \in G L(R)$, então $\tau_{1}\left(B A B^{-1}\right)=\tau_{1}(A)$. De fato, a proposição 3.5 nos garante que $B A B^{-1}=(B A) B^{-1}$ tem a mesma torção em $K_{1}(R)$ que $B^{-1}(B A)=A$.

Temos que $\langle G\rangle_{c, c}=\langle c / b\rangle\langle G\rangle_{b, b}\langle c / b\rangle^{-1}$, donde, pelo parágrafo anterior, $\tau\left(\langle G\rangle_{c, c}\right)=\tau\left(\langle G\rangle_{b, b}\right)$.

Temos também que, se $y=\bar{\delta} z+w$, com $z \in B_{i-1}$ e $w \in F_{2}$, então $G(y)=\delta d(\bar{\delta} z)+w=\delta z+w$. Mas, como $d(\delta z-\bar{\delta} z)=z-z=0$, temos que $x=\delta z-\bar{\delta} z \in B_{i}$, donde $\delta z=x+\bar{\delta} z$. Logo, $G(y)=\delta z+w=x+\bar{\delta} z+w=$ $x+y, x \in B_{i}$. Segue que $\langle G\rangle_{b, b}$ é da forma:

$$
\langle G\rangle_{b, b}=\begin{array}{r}
F_{1} \oplus B_{i} \\
\bar{\delta} B_{i-1} \oplus F_{2}
\end{array}\left(\begin{array}{cc}
F_{1} \oplus B_{i} & \bar{\delta} B_{i-1} \oplus F_{2} \\
X & 0
\end{array}\right)
$$

Claramente a torção desta matriz é nula.

Segue que $\tau(g)=\tau\left(\langle G\rangle_{c, c}\right)=\tau\left(\langle G\rangle_{b, b}\right)=0$, e portanto $g=1 \oplus \delta d$ é um isomorfismo simples.

Agora, suponha que $B_{i}$ e $B_{i-1}$ são módulos livres com bases $b_{i}$ e $b_{i-1}$, respectivamente. Então, $b_{i} \cup \delta b_{i-1}$ e $b_{i} \cup \bar{\delta} b_{i-1}$ são duas bases para $C_{i}$. Escreva $b_{i}=\left\{u_{1}, \ldots, u_{p}\right\}, b_{i-1}=\left\{v_{1}, \ldots, v_{q}\right\}$ e $b=b_{i} \cup \bar{\delta} b_{i-1}$.

Veja que $(1 \oplus \delta d)\left(u_{j}\right)=u_{j}$ e $(1 \oplus \delta d)\left(\bar{\delta} v_{k}\right)=\delta v_{k}$. Logo, $\langle 1 \oplus \delta d\rangle_{b, b}=$ $\left\langle b_{i} \cup \delta b_{i-1} / b_{i} \cup \bar{\delta} b_{i-1}\right\rangle$, donde $\tau\left(\left\langle b_{i} \cup \delta b_{i-1} / b_{i} \cup \bar{\delta} b_{i-1}\right\rangle\right)=0$.

Seja $c_{i}$ uma base para $C_{i}$, então temos $0=\tau\left(\left\langle b_{i} \cup \delta b_{i-1} / b_{i} \cup \bar{\delta} b_{i-1}\right\rangle\right)=$ $\tau\left(\left\langle b_{i} \cup \delta b_{i-1} / c_{i}\right\rangle\left\langle c_{i} / b_{i} \cup \bar{\delta} b_{i-1}\right\rangle\right)=\tau\left(\left\langle b_{i} \cup \delta b_{i-1} / c_{i}\right\rangle\right)+\tau\left(\left\langle c_{i} / b_{i} \cup \bar{\delta} b_{i-1}\right\rangle\right)=$ $\tau\left(\left\langle b_{i} \cup \delta b_{i-1} / c_{i}\right\rangle\right)+\tau\left(\left\langle b_{i} \cup \bar{\delta} b_{i-1} / c_{i}\right\rangle^{-1}\right)=\tau\left(\left\langle b_{i} \cup \delta b_{i-1} / c_{i}\right\rangle\right)-\tau\left(\left\langle b_{i} \cup \bar{\delta} b_{i-1} / c_{i}\right\rangle\right)$, mostrando assim que $\tau\left(\left\langle b_{i} \cup \delta b_{i-1} / c_{i}\right\rangle\right)=\tau\left(\left\langle b_{i} \cup \bar{\delta} b_{i-1} / c_{i}\right\rangle\right)$. 


\subsection{Equivalência estável de complexos de cadeia acíclicos}

Definição 3.14. Dizemos que um $(R, G)$-complexo $C$ é elementarmente trivial quando ele é da forma

$$
C: 0 \rightarrow C_{n} \stackrel{d}{\longrightarrow} C_{n-1} \rightarrow 0
$$

onde $d$ é um isomorfismo simples de $(R, G)$-módulos.

Observe que neste caso existem bases distinguidas para $C_{n}$ e $C_{n-1}$ de modo que a matriz de $d$ com relação a estas bases é a matriz identidade.

Definição 3.15. Dizemos que um $(R, G)$-complexo é trivial quando ele é a soma direta (de um número finito) de $(R, G)$-complexos elementarmente triviais.

Definição 3.16. Dizemos que dois $(R, G)$-complexos $C$ e $C^{\prime}$ são estavelmente equivalentes quando existem complexos triviais $T$ e $T^{\prime}$ de maneira que $C \oplus$ $T \cong_{S} C^{\prime} \oplus T^{\prime}$. Neste caso, denotamos $C \stackrel{s}{\sim} C^{\prime}$.

Proposição 3.17. Seja $C$ um $(R, G)$-complexo acíclico da forma

$$
C: 0 \rightarrow C_{n} \stackrel{d_{n}}{\longrightarrow} \cdots \stackrel{d_{i+3}}{\longrightarrow} C_{i+2} \stackrel{d_{i+2}}{\longrightarrow} C_{i+1} \stackrel{d_{i+1}}{\longrightarrow} C_{i} \rightarrow 0,
$$

com $n \geq i+1$, e seja $\delta: C \rightarrow C$ uma contração de cadeia. Então $C \stackrel{s}{\sim} C_{\delta}$, onde $C_{\delta}$ é o complexo

$$
\begin{gathered}
C_{\delta}: 0 \rightarrow C_{n} \stackrel{d_{n}}{\longrightarrow} \cdots \stackrel{d_{i+3}}{\longrightarrow} C_{i+2} \stackrel{d_{i+2}}{\longrightarrow} C_{i+1} \rightarrow 0 \\
\bigoplus C_{i} \overbrace{\delta_{i+1}}
\end{gathered}
$$

Demonstração. Considere os complexos triviais

$$
T: 0 \rightarrow T_{i+2} \stackrel{1}{\rightarrow} T_{i+1} \rightarrow 0
$$

e

$$
T^{\prime}: 0 \rightarrow T_{i+1}^{\prime} \stackrel{1}{\rightarrow} T_{i}^{\prime} \rightarrow 0
$$


onde $T_{i+2}=T_{i+1}=T_{i+1}^{\prime}=T_{i}^{\prime}=C_{i}$.

Mostremos que $C \oplus T \cong_{S} C_{\delta} \oplus T^{\prime}$.

Temos que $C \oplus T$ tem a forma

$$
C \oplus T: 0 \rightarrow C_{n} \stackrel{d_{n}}{\longrightarrow} \cdots \stackrel{d_{i+4}}{\longrightarrow} C_{i+3} \stackrel{d_{i+3}}{\longrightarrow} C_{i+2} \underset{d_{i+2} \oplus 1}{\oplus \underset{i+1}{\longrightarrow}} \stackrel{C^{0}}{C_{i}} C_{i} \longrightarrow 0
$$

Por sua vez, $C_{\delta} \oplus T^{\prime}$ tem a forma

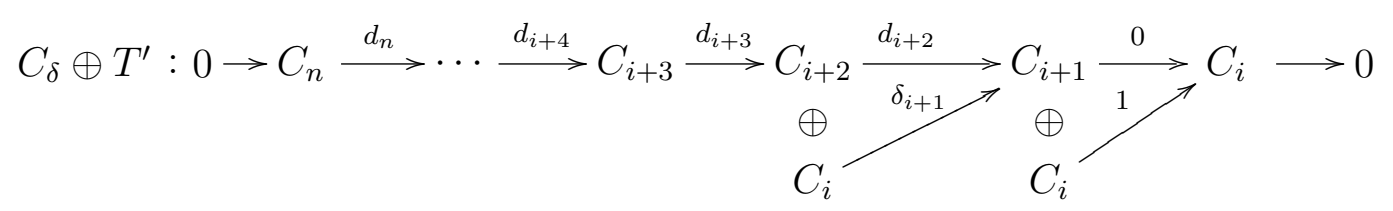

Defina $f: C \oplus T \rightarrow C_{\delta} \oplus T^{\prime}$ da seguinte maneira: $f_{j}=1$ se $j \neq i+1 \mathrm{e}$ $f_{i+1}\left(c_{i}+c_{i+1}\right)=d_{i+1} c_{i+1}+\left(\delta_{i+1} c_{i}+c_{i+1}\right)$, com $c_{i} \in C_{i}$ e $c_{i+1} \in C_{i+1}$.

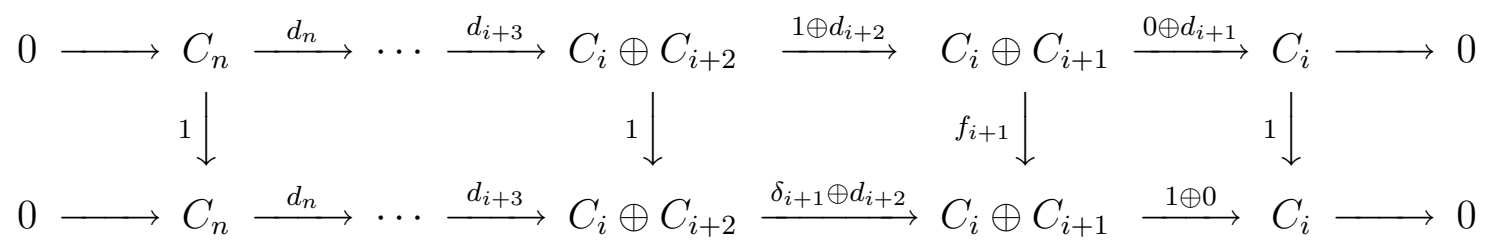

Claramente, $f_{j}$ é um isomorfismo simples para todo $j \neq i+1$.

Mostremos que $f_{i+1}$ é também um isomorfismo simples.

Verifica-se com facilidade que $f_{i+1}$ é um homomorfismo de módulos.

Tome $x \in C_{i}$ e $y \in C_{i+1}$. Note que, como $C$ é acíclico, $B_{i}=C_{i}$, donde $d_{i+1} \delta_{i+1}=1$ e $C_{i+1}=B_{i+1} \oplus \delta_{i+1} C_{i}$. Logo, $y$ tem a forma $z+\delta_{i+1} w$, com $z \in B_{i+1}$ e $w \in C_{i}$. Veja que $f_{i+1}\left((w-x)+\left(z+\delta_{i+1} x\right)\right)=d_{i+1}\left(z+\delta_{i+1} x\right)+$ $\left[\delta_{i+1}(w-x)+\left(z+\delta_{i+1} x\right)\right]=\left(d_{i+1} z+d_{i+1} \delta_{i+1} x\right)+\left(\delta_{i+1} w-\delta_{i+1} x+z+\delta_{i+1} x\right)=$ $x+\left(z+\delta_{i+1} w\right)=x+y$. Assim, $f_{i+1}$ é uma aplicação sobrejetiva.

Agora, supondo que $f_{i+1}(x+y)=0$, temos que $d_{i+1} y=0$ e $\delta_{i+1} x+y=0$. Aplicando $d_{i+1}$ nesta última igualdade, obtemos $x+d_{i+1} y=0, \operatorname{logo} x=0$, 
e então substituindo $x=0$ em $\delta_{i+1} x+y=0$, obtemos que $y=0$. Portanto, $f_{i+1}$ é injetiva.

Segue que, $f_{i+1}$ é um isomorfismo de módulos. Logo, podemos determinar sua torção em $K_{G}(R)$.

Temos que a matriz de $f_{i+1}$ é dada por

$$
\begin{aligned}
& C_{i} \quad C_{i+1} \\
& \left\langle f_{i+1}\right\rangle=\underset{C_{i+1}}{C_{i}}\left(\begin{array}{cc}
0 & \left\langle\delta_{i+1}\right\rangle \\
\left\langle d_{i+1}\right\rangle & I
\end{array}\right)=\left(\begin{array}{cc}
-I & \left\langle\delta_{i+1}\right\rangle \\
0 & I
\end{array}\right)\left(\begin{array}{cc}
I & 0 \\
\left\langle d_{i+1}\right\rangle & I
\end{array}\right)
\end{aligned}
$$

Pela proposição (3.9), temos que $\tau\left(\left\langle f_{i+1}\right\rangle\right)=0 \in K_{G} R$, o que finalmente mostra que $f_{i+1}$ é um isomorfismo simples.

Claramente, $f$ é uma aplicação de cadeia.

Mostramos, portanto, que $C \stackrel{s}{\sim} C_{\delta}$.

Verifica-se facilmente que o $(R, G)$-complexo $C_{\delta}$ construído acima é acíclico. Assim, podemos usar o resultado anterior indutivamente de forma a obter um $(R, G)$-complexo acíclico $C^{\prime}$ tal que $C \stackrel{s}{\sim} C^{\prime}$ e $C^{\prime}$ é 0 exceto em duas dimensões. O resultado a seguir mostra a forma de um $(R, G)$-complexo acíclico $C^{\prime}$ no caso de considerarmos uma contração $\delta$ que satisfaz $\delta^{2}=0$.

Proposição 3.18. Seja $C$ um $(R, G)$-complexo acíclico com operador bordo $d$ e contração $\delta$ satisfazendo $\delta^{2}=0$. Considere os seguintes $(R, G)$-módulos

$$
\begin{gathered}
C_{\text {impar }}=C_{1} \oplus C_{3} \oplus C_{5} \oplus \ldots \\
C_{\text {par }}=C_{0} \oplus C_{2} \oplus C_{4} \oplus \ldots
\end{gathered}
$$


e o isomorfismo $(d+\delta)_{\text {impar }}: C_{\text {impar }} \rightarrow C_{\text {par }}$ dado por

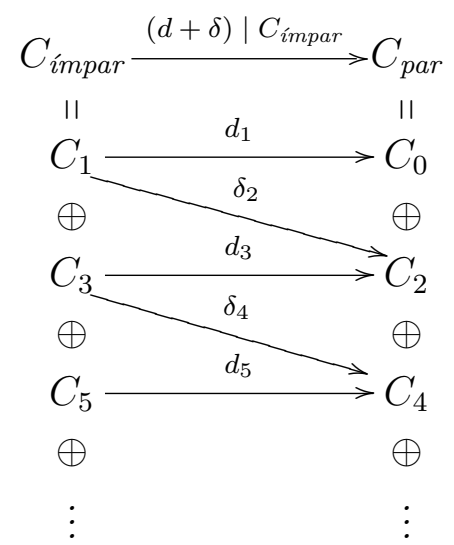

Seja $C^{\prime}$ o $(R, G)$-complexo

$$
C^{\prime}: 0 \rightarrow C_{m}^{\prime}=C_{\text {impar }} \stackrel{(d+\delta)_{\text {impar }}}{\longrightarrow} C_{m-1}^{\prime}=C_{\text {par }} \rightarrow 0,
$$

para algum natural ímpar $m$. Então $C \stackrel{s}{\sim} C^{\prime}$.

Demonstração. Seja $m$ um natural ímpar tal que o complexo $C$ tem a forma

$$
0 \rightarrow C_{m} \stackrel{d_{m}}{\longrightarrow} C_{m-1} \stackrel{d_{m-1}}{\longrightarrow} \cdots \rightarrow C_{1} \stackrel{d_{1}}{\longrightarrow} C_{0} \rightarrow 0 .
$$

Não estamos excluindo o caso em que $C_{m}=0$.

Para $j \leq m$, defina $C_{j}^{\prime}=C_{j} \oplus C_{j-2} \oplus C_{j-4} \oplus \ldots$

Note que $C_{m}^{\prime}=C_{\text {ímpar }}$ e $C_{m-1}^{\prime}=C_{\text {par }}$.

Seja $D^{i}$ o complexo de cadeia dado por

$$
0 \rightarrow C_{m} \stackrel{d_{m}}{\longrightarrow} \cdots \rightarrow C_{i+2} \stackrel{d_{i+2}}{\longrightarrow} C_{i+1}^{\prime} \stackrel{d^{\prime}}{\longrightarrow} C_{i}^{\prime} \rightarrow 0,
$$

onde $d^{\prime}$ é definido por

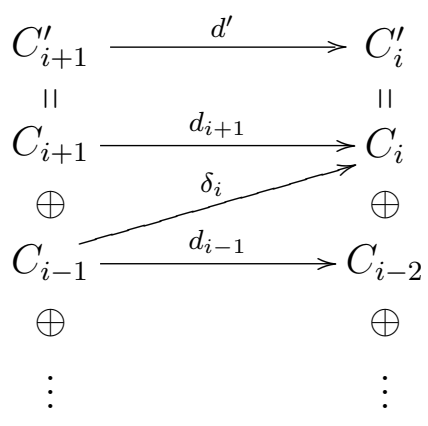


Note que $D^{0}=C$ e $D^{m-1}=C^{\prime}$.

Defina $\Delta^{i}=\Delta: D^{i} \rightarrow D^{i}$ da seguinte maneira

$$
\Delta_{j+1}=\delta_{j+1}: C_{j} \rightarrow C_{j+1}, \quad \text { se } \quad j \geq i+2,
$$

$\Delta_{i+2}=\delta_{i+2} \pi, \quad$ onde $\pi: C_{i+1}^{\prime} \rightarrow C_{i+1} \quad$ é a projeção natural e $\Delta_{i+1}: C_{i}^{\prime} \rightarrow C_{i+1}^{\prime}$ é dada por

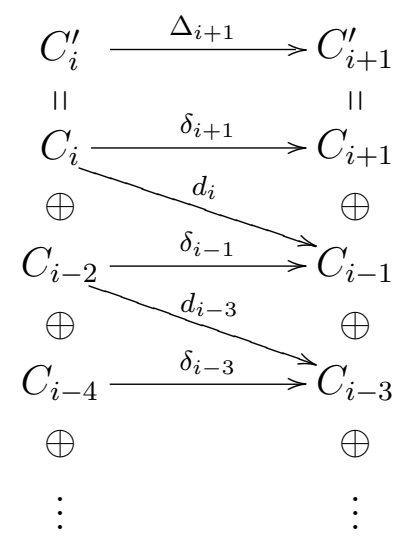

Verifica-se sem dificuldades que $\Delta^{i}$ é uma contração de cadeia para $D^{i}$.

Agora, $D^{i}$ e $\Delta=\Delta^{i}$ satisfazem as condições da proposição (3.17), logo $D^{i} \stackrel{s}{\sim} D_{\Delta}^{i}$, mas $D_{\Delta}^{i}=D^{i+1}$.

Portanto, por indução, obtemos que $C=D^{0} \stackrel{s}{\sim} D^{m-1}=C^{\prime}$.

\subsection{Torção de um $(R, G)$-complexo acíclico}

Seja $C$ um $(R, G)$-complexo acíclico com operador bordo $d$. Tome arbitrariamente uma contração de cadeia $\delta: C \rightarrow C$. Como antes, sejam $C_{\text {ímpar }}=C_{1} \oplus C_{3} \oplus \ldots$ e $C_{\text {par }}=C_{0} \oplus C_{2} \oplus \ldots$ Considere novamente a

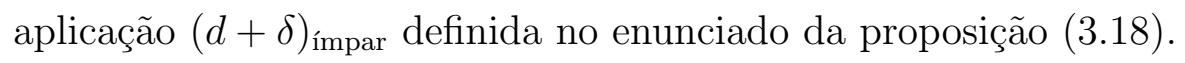

Nesta seção mostraremos que esta aplicação é um isomorfismo cuja torção em $K_{G}(R)$ não se altera quando substituímos a contração de cadeia $\delta$ por 
uma outra contração de cadeia $\bar{\delta}$ de $C$ ou quando escolhemos bases diferentes de uma mesma família distinguida.

Para simplificar a notação vamos escrever apenas $(d+\delta)$ para indicar $(d+\delta)_{\text {ímpar }}$, quando o contexto deixar claro a aplicação em questão.

Escolha uma base $c_{i}$ para cada $C_{i}$ e denote $c_{\text {ímpar }}=\bigcup_{i} c_{2 i+1}, c_{\mathrm{par}}=\bigcup_{i} c_{2 i}$ e $c=\bigcup_{i} c_{i}$ as bases correspondentes para $C_{\text {ímpar }}, C_{\text {par }}$ e $C$.

Para facilitar, denotaremos $\langle d+\delta\rangle_{c_{\text {impar }}, c_{\text {par }}}$ por $\langle d+\delta\rangle_{c}$ ou por $\langle d+\delta\rangle$ quando não houver dúvidas sobre a base usada.

Proposição 3.19. Defina o homomorfismo de módulos $(d+\delta)_{p a r}: C_{p a r} \rightarrow$ $C_{\text {impar }}$ de maneira análoga ao homomorfismo $(d+\delta)_{\text {impar. }}$ Então $\left\langle(d+\delta)_{\text {impar }}\right\rangle_{c}$ $e\left\langle(d+\delta)_{\text {par }}\right\rangle_{c}$ são matrizes não-singulares com $\tau\left(\left\langle(d+\delta)_{\text {impar }}\right\rangle_{c}\right)=-\tau(\langle(d+$ $\left.\left.\delta)_{\text {par }}\right\rangle_{c}\right)$.

Demonstração. Facilmente prova-se que

$$
\begin{aligned}
& \begin{array}{cccc}
C_{1} & C_{3} & C_{5} & \ldots
\end{array} \\
& \begin{aligned}
& C_{1} \\
\left\langle(d+\delta)_{\text {impar }}\right\rangle_{c}\left\langle(d+\delta)_{\text {par }}\right\rangle_{c}= & C_{3} \\
C_{5} & \vdots
\end{aligned}\left(\begin{array}{cccc}
I & \left\langle\delta_{3} \delta_{2}\right\rangle & 0 & \cdots \\
0 & I & \left\langle\delta_{5} \delta_{4}\right\rangle & 0 \\
0 & 0 & I & \left\langle\delta_{7} \delta_{6}\right\rangle \\
\vdots & \vdots & & \ddots
\end{array}\right)
\end{aligned}
$$

e que

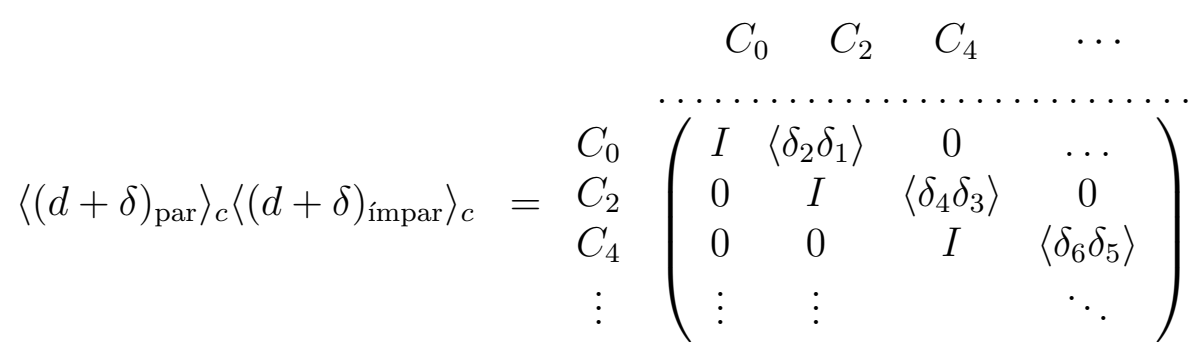

Note que, pela proposição 3.9 na página 35, temos que ambas as matrizes do lado direito das igualdades acima são não-singulares e têm torção nula, o que mostra a validade da proposição. 
Proposição 3.20. Para cada $i$, sejam $c_{i}$ e $c_{i}^{\prime}$ bases arbitrárias para $C_{i}$. Sejam $c=\bigcup_{i} c_{i}$ e $c^{\prime}=\bigcup_{i} c_{i}^{\prime}$ bases para $C$. Então temos que

$$
\tau\left(\left\langle(d+\delta)_{\text {impar }}\right\rangle_{c}\right)=\tau\left(\left\langle(d+\delta)_{\text {impar }_{c^{\prime}}}\right)+\sum_{i}(-1)^{i} \tau\left(\left\langle c_{i}^{\prime} / c_{i}\right\rangle\right) .\right.
$$

Demonstração. Temos que $\left\langle(d+\delta)_{\text {ímpar }}\right\rangle_{c}=\langle d+\delta\rangle_{c}=\left\langle c_{\text {ímpar }} / c_{\text {impar }}^{\prime}\right\rangle\langle d+$ $\delta\rangle_{c^{\prime}}\left\langle c_{\text {par }}^{\prime} / c_{\text {par }}\right\rangle$, donde $\tau\left(\langle d+\delta\rangle_{c}\right)=\tau\left(\left\langle c_{\text {ímpar }} / c_{\text {impar }}^{\prime}\right\rangle\right)+\tau\left(\langle d+\delta\rangle_{c^{\prime}}\right)+$ $\tau\left(\left\langle c_{\text {par }}^{\prime} / c_{\text {par }}\right\rangle\right)$.

Mas,

$$
\left\langle c_{\text {impar }} / c_{\text {impar }}^{\prime}\right\rangle=\left(\begin{array}{cccc}
\left\langle c_{1} / c_{1}^{\prime}\right\rangle & 0 & \ldots & 0 \\
0 & \left\langle c_{3} / c_{3}^{\prime}\right\rangle & 0 & \ldots \\
0 & \ldots & \left\langle c_{5} / c_{5}^{\prime}\right\rangle & 0 \\
\vdots & & & \ddots
\end{array}\right),
$$

logo, usando mais uma vez a proposição 3.9$], \tau\left(\left\langle c_{\text {ímpar }} / c_{\text {impar }}^{\prime}\right\rangle\right)=$ $\sum_{k} \tau\left(\left\langle c_{2 k+1} / c_{2 k+1}^{\prime}\right\rangle\right)=\sum_{k}-\tau\left(\left\langle c_{2 k+1}^{\prime} / c_{2 k+1}\right\rangle\right)$.

De maneira análoga mostramos que $\tau\left(\left\langle c_{\mathrm{par}}^{\prime} / c_{\mathrm{par}}\right\rangle\right)=\sum_{k} \tau\left(\left\langle c_{2 k}^{\prime} / c_{2 k}\right\rangle\right)$.

O resultado segue destas três igualdades.

Veja que se, em particular, escolhermos bases $c_{i}$ e $c_{i}^{\prime}$ distinguidas para cada $C_{i}$, então obtemos a igualdade $\tau\left(\left\langle(d+\delta)_{\text {impar }}\right\rangle_{c}\right)=\tau\left(\left\langle(d+\delta)_{\text {ímpar }}\right\rangle_{c^{\prime}}\right)$.

Proposição 3.21. Sejam $\delta$ e $\bar{\delta}$ duas contrações de cadeia para o $(R, G)$ complexo acíclico $C$. Então $\tau(d+\delta)=\tau(d+\bar{\delta})$, onde as bases consideradas são distinguidas.

Demonstração. Temos que $\tau(d+\bar{\delta})-\tau(d+\delta)=\tau\left((d+\bar{\delta})_{\text {impar }}\right)-\tau((d+$ $\left.\delta)_{\text {impar }}\right)=\tau\left(\left\langle(d+\bar{\delta})_{\text {impar }}\right\rangle\right)+\tau\left(\left\langle(d+\delta)_{\text {par }}\right\rangle\right)=\tau\left(\left\langle(d+\bar{\delta})_{\text {impar }}\right\rangle\left\langle(d+\delta)_{\text {par }}\right\rangle\right)=$ $\tau\left(\left\langle(d+\delta)_{\mathrm{par}}(d+\bar{\delta})_{\text {ímpar }}\right\rangle\right)=\tau\left((d+\delta)_{\mathrm{par}}(d+\bar{\delta})_{\text {ímpar }}\right)=\tau\left((\delta d+d \bar{\delta}+\delta \bar{\delta})_{\text {ímpar }}\right)$. 
Verifica-se facilmente que

$$
\begin{aligned}
& \begin{array}{llll}
C_{1} & C_{3} & C_{5} & \ldots
\end{array} \\
& \left\langle(\delta d+d \bar{\delta}+\delta \bar{\delta})_{\text {impar }}\right\rangle=C_{1}\left(\begin{array}{ccccc}
\left\langle\delta_{1} d_{1}+d_{2} \bar{\delta}_{1}\right\rangle & \left\langle\delta_{3} \bar{\delta}_{2}\right\rangle & 0 & \ldots & 0 \\
0 & \left\langle\delta_{3} d_{3}+d_{4} \bar{\delta}_{3}\right\rangle & \left\langle\delta_{5} \bar{\delta}_{4}\right\rangle & 0 & \ldots \\
\vdots & & \ddots & \ddots
\end{array}\right)
\end{aligned}
$$

Então se cada matriz $\left\langle\delta_{2 i+1} d_{2 i+1}+d_{2 i+2} \bar{\delta}_{2 i+1}\right\rangle$ é não-singular, temos que a matriz acima é não-singular e que sua torção é igual a $\sum_{i} \tau\left(\left\langle\delta_{2 i+1} d_{2 i+1}+\right.\right.$ $\left.\left.d_{2 i+2} \bar{\delta}_{2 i+1}\right\rangle\right)$.

Mas, por cálculo direto, obtemos que $\left(\delta_{i} d_{i}+d_{i+1} \bar{\delta}_{i}\right): C_{i}=B_{i} \oplus \bar{\delta}_{i} B_{i-1} \rightarrow$ $C_{i}=B_{i} \oplus \delta_{i} B_{i-1}$ coincide com a aplicação $1 \oplus \delta d$ já apresentada no lema 3.16) da página 47 .

Este lema nos fornece que cada $\left\langle\delta_{2 i+1} d_{2 i+1}+d_{2 i+2} \bar{\delta}_{2 i+1}\right\rangle$ é não-singular e sua torção é nula.

Portanto, $\tau\left((\delta d+d \bar{\delta}+\delta \bar{\delta})_{\text {ímpar }}\right)=0$, donde $\tau(d+\bar{\delta})=\tau(d+\delta)$.

Esta última proposição mostra que a torção do isomorfismo $(d+\delta)_{\text {ímpar de }}$ fato independe da escolha das bases distinguidas consideradas e da contração de cadeia $\delta$ de $C$, o que torna a definição a seguir é coerente.

Definição 3.17. Seja $C$ um $(R, G)$-complexo acíclico com contração de cadeia $\delta$. Definimos a torção de $C$ e escrevemos $\tau(C)$ como sendo o elemento $\tau\left((d+\delta)_{\text {impar }}\right) \in K_{G}(R)$.

Observe que

$$
\begin{aligned}
& \begin{array}{cccc}
C_{0} & C_{2} & C_{4} & \ldots
\end{array} \\
& \langle d+\delta\rangle=\begin{array}{c}
C_{1} \\
C_{3} \\
C_{5} \\
\vdots
\end{array}\left(\begin{array}{cccc}
\left\langle d_{1}\right\rangle & \left\langle\delta_{2}\right\rangle & 0 & \cdots \\
0 & \left\langle d_{3}\right\rangle & \left\langle\delta_{4}\right\rangle & 0 \\
0 & 0 & \left\langle d_{5}\right\rangle & \left\langle\delta_{6}\right\rangle \\
\vdots & \vdots & & \ddots
\end{array}\right)
\end{aligned}
$$


Contudo, devemos ficar atentos para não cometer o erro de achar que a torção desta matriz é igual a soma $\sum_{i} \tau\left(d_{2 i+1}\right)$, pois não temos a garantia de que cada $d_{2 i+1}$ é um isomorfismo.

\subsection{Caracterização da torção de um $(R, G)$-complexo acíclico}

Sejam $C$ e $C^{\prime}$ dois $(R, G)$-complexos acíclicos.

Considere as propriedades:

P1: $C \cong{ }_{S} C^{\prime} \Rightarrow \tau(C)=\tau\left(C^{\prime}\right)$.

P2: $\tau\left(C \oplus C^{\prime}\right)=\tau(C)+\tau\left(C^{\prime}\right)$.

P3: $\tau\left(0 \rightarrow C_{n} \stackrel{d_{n}}{\longrightarrow} C_{n-1} \rightarrow 0\right)=(-1)^{n-1} \tau(d)$.

Nesta seção mostraremos que a torção de um $(R, G)$-complexo acíclico está completamente caracterizada por estas propriedades, ou seja, a torção de complexos definida na seção anterior satisfaz estas propriedades e se existe uma outra aplicação bem definida que associa a cada $(R, G)$-complexo acíclico um elemento de $K_{G}(R)$ e que satisfaz estas propriedades, então esta aplicação é a torção que conhecemos.

Teorema 3.22. Se $\mathcal{C}$ é a classe dos $(R, G)$-complexos acíclicos, então a aplicação torção $\tau: \mathcal{C} \rightarrow K_{G}(R)$ definida na seção anterior satisfaz as propriedades $\boldsymbol{P} \mathbf{1}, \boldsymbol{P} 2$ e $\boldsymbol{P} 3$.

Demonstração. Sejam $C$ e $C^{\prime}(R, G)$-complexos acíclicos tais que $f: C \cong_{S}$ $C^{\prime}$. Então, pela proposição 3.12 , existem bases distinguidas para cada $C_{i}$ e para cada $C_{i}^{\prime}$ de forma cada matriz $\left\langle f_{i}\right\rangle$ é a identidade e, se $d$ e $d^{\prime}$ são os operadores bordo para $C$ e $C^{\prime}$, respectivamente, então $\left\langle d_{i}\right\rangle=\left\langle d_{i}^{\prime}\right\rangle, \forall i$.

Seja $\delta$ uma contração de cadeia para $C$. Defina $\delta^{\prime}=f \delta f^{-1}$. Afirmamos que $\delta^{\prime}$ é uma contração de cadeia para $C^{\prime}$. De fato, observe que $\left\langle\delta^{\prime}\right\rangle=\left\langle f \delta f^{-1}\right\rangle=$ 
$\left\langle f^{-1}\right\rangle\langle\delta\rangle\langle f\rangle=\langle f\rangle^{-1}\langle\delta\rangle\langle f\rangle=\langle\delta\rangle$, donde $\left\langle d^{\prime} \delta^{\prime}+\delta^{\prime} d^{\prime}\right\rangle=\left\langle d^{\prime} \delta^{\prime}\right\rangle+\left\langle\delta^{\prime} d^{\prime}\right\rangle=$ $\left\langle\delta^{\prime}\right\rangle\left\langle d^{\prime}\right\rangle+\left\langle d^{\prime}\right\rangle\left\langle\delta^{\prime}\right\rangle=\langle\delta\rangle\langle d\rangle+\langle d\rangle\langle\delta\rangle=\langle d \delta+\delta d\rangle=I$, o que prova a afirmação feita.

Agora, veja que

$\left\langle d^{\prime}+\delta^{\prime}\right\rangle=\left(\begin{array}{cccc}\left\langle d_{1}^{\prime}\right\rangle & \left\langle\delta_{2}^{\prime}\right\rangle & 0 & \ldots \\ 0 & \left\langle d_{3}^{\prime}\right\rangle & \left\langle\delta_{4}^{\prime}\right\rangle & 0 \\ 0 & 0 & \left\langle d_{5}^{\prime}\right\rangle & \left\langle\delta_{6}^{\prime}\right\rangle \\ \vdots & \vdots & & \ddots\end{array}\right)=\left(\begin{array}{cccc}\left\langle d_{1}\right\rangle & \left\langle\delta_{2}\right\rangle & 0 & \ldots \\ 0 & \left\langle d_{3}\right\rangle & \left\langle\delta_{4}\right\rangle & 0 \\ 0 & 0 & \left\langle d_{5}\right\rangle & \left\langle\delta_{6}\right\rangle \\ \vdots & \vdots & & \ddots\end{array}\right)=\langle d+\delta\rangle$.

Assim, $\tau\left(C^{\prime}\right)=\tau\left(\left\langle d^{\prime}+\delta^{\prime}\right\rangle\right)=\tau(\langle d+\delta\rangle)=\tau(C)$, e então $\mathbf{P} 1$ está verificada.

Sejam $C^{\prime}$ e $C^{\prime \prime}$ dois $(R, G)$-complexos acíclicos com operadores bordo $d^{\prime}$ e $d^{\prime \prime}$ e contrações de cadeia $\delta^{\prime}$ e $\delta^{\prime \prime}$, respectivamente. Temos então que $C=C^{\prime} \oplus C^{\prime \prime}$ é um $(R, G)$-complexo acíclico com operador bordo $d=d^{\prime} \oplus d^{\prime \prime}$ e contração de cadeia $\delta=\delta^{\prime} \oplus \delta^{\prime \prime}$.

Observe que

$$
\left\langle d_{i}\right\rangle=\left(\begin{array}{cc}
\left\langle d_{i}^{\prime}\right\rangle & 0 \\
0 & \left\langle d_{i}^{\prime \prime}\right\rangle
\end{array}\right)
$$

e que

$$
\left\langle\delta_{i}\right\rangle=\left(\begin{array}{cc}
\left\langle\delta_{i}^{\prime}\right\rangle & 0 \\
0 & \left\langle\delta_{i}^{\prime \prime}\right\rangle
\end{array}\right)
$$

Assim,

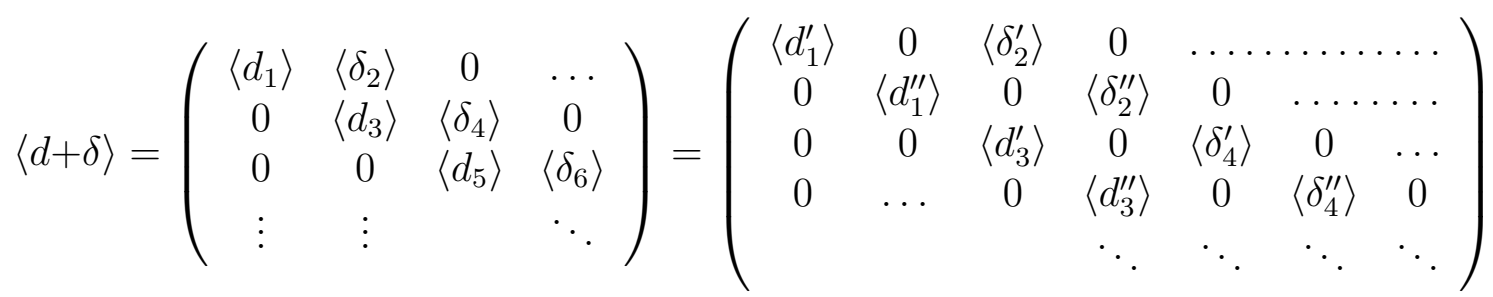

Agora, através de troca de linhas e colunas, obtemos que esta matriz tem a mesma torção que

$$
\left(\begin{array}{cc}
\left\langle d^{\prime}+\delta^{\prime}\right\rangle & 0 \\
0 & \left\langle d^{\prime \prime}+\delta^{\prime \prime}\right\rangle
\end{array}\right) .
$$

Segue que

$$
\begin{aligned}
\tau(C)=\tau(\langle d+\delta\rangle) & =\tau\left(\begin{array}{cc}
\left\langle d^{\prime}+\delta^{\prime}\right\rangle & 0 \\
0 & \left\langle d^{\prime \prime}+\delta^{\prime \prime}\right\rangle
\end{array}\right) \\
& =\tau\left(\left\langle d^{\prime}+\delta^{\prime}\right\rangle\right)+\tau\left(\left\langle d^{\prime \prime}+\delta^{\prime \prime}\right\rangle\right)=\tau\left(C^{\prime}\right)+\tau\left(C^{\prime \prime}\right),
\end{aligned}
$$


o que mostra que a propriedade $\mathbf{P} 2$ é válida.

Por fim, mostremos que P3 é também satisfeita.

Seja $C$ um $(R, G)$-complexo acíclico da forma

$$
0 \rightarrow C_{n} \stackrel{d}{\longrightarrow} C_{n-1} \rightarrow 0 .
$$

Claramente, $d$ é um isomorfismo. Defina $\delta=d^{-1}$. Evidentemente, $\delta$ é uma contração de cadeia para $C$.

Suponha que $n$ é ímpar. Então temos:

$$
\begin{gathered}
C_{\text {ímpar }} \stackrel{d+\delta}{\longrightarrow} C_{\text {par }} \\
{ }^{\text {।I }} \stackrel{\text { । }}{ } C_{n-1} \stackrel{d}{\longrightarrow} C_{n-1}
\end{gathered}
$$

Logo, $\langle d+\delta\rangle=\langle d\rangle$, donde $\tau(C)=(-1)^{n-1} \tau(\langle d\rangle)$.

Agora, suponha que $n$ é par. Então:

$$
\begin{gathered}
C_{\text {ímpar }} \stackrel{d+\delta}{\longrightarrow} C_{\text {par }} \\
C_{n-1} \stackrel{\text { ।I }}{\longrightarrow} C_{n}
\end{gathered}
$$

Assim, $\langle d+\delta\rangle=\langle\delta\rangle=\left\langle d^{-1}\right\rangle=\langle d\rangle^{-1}$ donde $\tau(C)=\tau\left(\langle d\rangle^{-1}\right)=-\tau(\langle d\rangle)=$ $(-1)^{n-1} \tau(\langle d\rangle)$.

Proposição 3.23. Sejam $C, C^{\prime}$ e $C^{\prime \prime}(R, G)$-complexos acíclicos com bases distinguidas $c, c^{\prime}$ e $c^{\prime \prime}$, respectivamente. Suponha que

$$
0 \rightarrow C^{\prime} \stackrel{i}{\rightarrow} C \stackrel{j}{\rightarrow} C^{\prime \prime} \rightarrow 0
$$

é uma seqüência exata curta de complexos de cadeia e que $\sigma: C^{\prime \prime} \rightarrow C$ é uma seção arbitrária. Então

$$
\tau(C)=\tau\left(C^{\prime}\right)+\tau\left(C^{\prime \prime}\right)+\sum_{k}(-1)^{k} \tau\left(\left\langle c_{k}^{\prime} c_{k}^{\prime \prime} / c_{k}\right\rangle\right),
$$

onde $c_{k}^{\prime} c_{k}^{\prime \prime}$ denota a base $i\left(c_{k}^{\prime}\right) \cup \sigma\left(c_{k}^{\prime \prime}\right)$ de $C_{k}$. Se, em particular, a base $i\left(c_{k}^{\prime}\right) \cup$ $\sigma\left(c_{k}^{\prime \prime}\right)$ é base distinguida para todo $k$, então $\tau(C)=\tau\left(C^{\prime}\right)+\tau\left(C^{\prime \prime}\right)$. 
Demonstração. A proposição 3.15 da página 47, nos garante a existência de uma seção $s: C^{\prime \prime} \rightarrow C$ de forma que $s$ é uma aplicação de cadeia e $(i \oplus s): C^{\prime} \oplus C^{\prime \prime} \rightarrow C=i\left(C^{\prime}\right) \oplus s\left(C^{\prime \prime}\right)$ é um isomorfismo de cadeia.

Temos então que $i\left(c_{k}^{\prime}\right) \cup s\left(c_{k}^{\prime \prime}\right)$ é uma base para $C_{k}$, onde $c_{k}^{\prime}$ e $c_{k}^{\prime \prime}$ são bases distinguidas para $C_{k}^{\prime}$ e $C_{k}^{\prime \prime}$. Cabe ressaltar que a base $i\left(c_{k}^{\prime}\right) \cup s\left(c_{k}^{\prime \prime}\right)$ não é necessariamente distinguida.

Seja $C^{\gamma}$ o $(R, G)$-complexo constituído do complexo de cadeia $C$, onde cada $C_{k}$ tem como base distinguida $\gamma_{k}=i\left(c_{k}^{\prime}\right) \cup s\left(c_{k}^{\prime \prime}\right)$.

Observe que $(i \oplus s): C^{\prime} \oplus C^{\prime \prime} \cong{ }_{S} C^{\gamma}$.

Então, as propriedades $\mathbf{P} 1$ e $\mathbf{P} 2$ nos garantem que $\tau\left(C^{\gamma}\right)=\tau\left(C^{\prime}\right)+\tau\left(C^{\prime \prime}\right)$.

Seja $\delta$ um contração de cadeia para $C$, então claramente $\delta$ é uma contração de cadeia para $C^{\gamma}$.

Agora, pela proposição 3.20 , temos:

$\tau(C)=\tau\left(\langle d+\delta\rangle_{c}\right)=\tau\left(\langle d+\delta\rangle_{\gamma}\right)+\sum_{k}(-1)^{k} \tau\left(\left\langle\gamma_{k} / c_{k}\right\rangle\right)=\tau\left(C^{\gamma}\right)+\sum_{k}(-1)^{k} \tau\left(\left\langle\gamma_{k} / c_{k}\right\rangle\right)$

Assim, resta apenas mostrar que $\tau\left(\left\langle c_{k}^{\prime} c_{k}^{\prime \prime} / c_{k}\right\rangle\right)=\tau\left(\left\langle\gamma_{k} / c_{k}\right\rangle\right)$.

Observe que se $y \in C_{k}^{\prime \prime}$, então $j_{k}\left(\sigma_{k}(y)-s_{k}(y)\right)=0$, donde $\left(\sigma_{k}(y)-\right.$ $\left.s_{k}(y)\right) \in \operatorname{Ker}\left(j_{k}\right)$. Mas $\operatorname{Ker}\left(j_{k}\right)=i_{k}\left(C_{k}^{\prime}\right)$, logo $\sigma_{k}(y)-s_{k}(y)=i_{k}(x)$, para algum $x \in C_{k}^{\prime}$. Assim $\sigma_{k}(y)=i_{k}(x)+s_{k}(y)$. Segue que a matriz $\left\langle c_{k}^{\prime} c_{k}^{\prime \prime} / \gamma_{k}\right\rangle$ tem a forma:

$$
\left(\begin{array}{ll}
I & 0 \\
X & I
\end{array}\right)
$$

que por (3.9) é não-singular e tem torção nula.

Agora, $\left\langle c_{k}^{\prime} c_{k}^{\prime \prime} / c_{k}\right\rangle=\left\langle c_{k}^{\prime} c_{k}^{\prime \prime} / \gamma_{k}\right\rangle\left\langle\gamma_{k} / c_{k}\right\rangle, \operatorname{logo} \tau\left(\left\langle c_{k}^{\prime} c_{k}^{\prime \prime} / c_{k}\right\rangle\right)=\tau\left(\left\langle c_{k}^{\prime} c_{k}^{\prime \prime} / \gamma_{k}\right\rangle\right)+$ $\tau\left(\left\langle\gamma_{k} / c_{k}\right\rangle\right)=\tau\left(\left\langle\gamma_{k} / c_{k}\right\rangle\right)$

Observe que a proposição acima é uma versão mais geral para a propriedade $\mathbf{P 2}$. 
Proposição 3.24. A aplicação torção $\tau: \mathcal{C} \rightarrow K_{G}(R)$ é a única função que satisfaz as propriedades $\boldsymbol{P} \mathbf{1}, \boldsymbol{P} 2$ e $\boldsymbol{P} 3$.

Demonstração. No primeiro resultado desta seção vimos que a aplicação torção $\tau: \mathcal{C} \rightarrow K_{G}(R)$ de fato é uma função que satisfaz as propriedades P1, P2 e P3.

Suponha que $\mu: \mathcal{C} \rightarrow K_{G}(R)$ também é uma função que satisfaz estas propriedades.

Tome $C \in \mathcal{C}$. Pelo resultado $3.18, C \stackrel{s}{\sim} C^{\prime}$, onde $C^{\prime}$ é da forma

$$
0 \rightarrow C_{m}^{\prime} \stackrel{d^{\prime}}{\longrightarrow} C_{m-1}^{\prime} \rightarrow 0
$$

Então existem complexos triviais $T$ e $T^{\prime}$ tais que $C \oplus T \cong_{s} C^{\prime} \oplus T^{\prime}$.

Assim, pelas propriedades $\mathbf{P} 1$ e P2, temos $\tau(C)+\tau(T)=\tau(C \oplus T)=$ $\tau\left(C^{\prime} \oplus T^{\prime}\right)=\tau\left(C^{\prime}\right)+\tau\left(T^{\prime}\right)$.

Da mesma forma, temos que $\mu(C)+\mu(T)=\mu\left(C^{\prime}\right)+\mu\left(T^{\prime}\right)$.

Agora, seja $\bar{C}$ um complexo elementarmente trivial, isto é, um $(R, G)$ complexo da forma

$$
0 \rightarrow \bar{C}_{n} \stackrel{\bar{d}}{\longrightarrow} \bar{C}_{n-1} \rightarrow 0
$$

onde $\bar{d}$ é um isomorfismo simples.

Segue que como $\tau$ e $\mu$ satisfazem a propriedade P3, $\mu(\bar{C})=\tau(\bar{C})=$ $(-1)^{n-1} \tau(\langle\bar{d}\rangle)=0$.

Assim, como todo complexo trivial é a soma direta (de um número finito) de complexos elementarmente triviais e como $\tau$ e $\mu$ satisfazem P2, $\tau$ e $\mu$ destes complexos também são nulos.

Logo, $\tau(C)=\tau\left(C^{\prime}\right)$ e $\mu(C)=\mu\left(C^{\prime}\right)$.

Mas, $\tau\left(C^{\prime}\right)=(-1)^{m-1} \tau\left(\left\langle d^{\prime}\right\rangle\right)=\mu\left(C^{\prime}\right)$, pela propriedade P3.

Portanto, $\mu(C)=\mu\left(C^{\prime}\right)=\tau\left(C^{\prime}\right)=\tau(C)$. 
Proposição 3.25. Seja $\mathcal{C}_{0}$ o conjunto das classes de equivalência da relação $\stackrel{s}{\sim}$. Denote a classe de equivalência de um $(R, G)$-complexo acíclico $C$ por $[C] . E m \mathcal{C}_{0}$ considere a operação

$$
[C]+\left[C^{\prime}\right]=\left[C \oplus C^{\prime}\right]
$$

$\mathcal{C}_{0}$ com esta operação é um semigrupo. Seja $\tau_{0}: \mathcal{C}_{0} \rightarrow K_{G}(R)$ dada por

$$
\tau_{0}[C]=\tau(C)
$$

Então $\mathcal{C}_{0}$ é um grupo e $\tau_{0}$ é um isomorfismo de grupos.

Demonstração. Facilmente prova-se que a operação $[C]+\left[C^{\prime}\right]=\left[C \oplus C^{\prime}\right]$ está bem definida e que vale a associatividade. Logo, $\mathcal{C}_{0}$ é de fato um semigrupo com esta operação.

Agora, se $\tau_{0}: \mathcal{C}_{0} \rightarrow K_{G}(R)$ é um isomorfismo de semigrupos, então $\mathcal{C}_{0}$ é, assim como $K_{G}(R)$, um grupo.

Suponha que $C \stackrel{s}{\sim} C^{\prime}$, então existem complexos triviais $T$ e $T^{\prime}$ tais que $C \oplus$ $T \cong{ }_{S} C^{\prime} \oplus T^{\prime}$. Assim, pelo teorema 3.22, temos $\tau(C)+\tau(T)=\tau\left(C^{\prime}\right)+\tau\left(T^{\prime}\right)$. Mas, pela demonstração da proposição anterior, temos que $\tau(T)=\tau\left(T^{\prime}\right)=0$, donde $\tau(C)=\tau\left(C^{\prime}\right)$, mostrando que $\tau_{0}$ está realmente bem definida.

Observe que $\tau_{0}\left(\left[C \oplus C^{\prime}\right]\right)=\tau\left(C \oplus C^{\prime}\right)=\tau(C)+\tau\left(C^{\prime}\right)=\tau_{0}[C]+\tau_{0}\left[C^{\prime}\right]$, $\operatorname{logo} \tau_{0}$ é um homomorfismo de semigrupos.

Resta apenas provar que $\tau_{0}$ é bijetiva, mas esta demonstração não apresenta dificuldades e por isso será omitida.

\subsection{Mudança de anéis}

Sejam $(R, G)$ e $\left(R^{\prime}, G^{\prime}\right)$ pares tais que $R$ e $R^{\prime}$ são anéis que satisfazem $\left(^{*}\right)$ e $G$ e $G^{\prime}$ são subgrupos dos grupos das unidades de $R$ e $R^{\prime}$ que contêm -1 e $-1^{\prime}$, respectivamente. 
Sejam $h: R \rightarrow R^{\prime}$ um homomorfismo de anéis satisfazendo $h(G) \subset G^{\prime}$ e $C$ um $(R, G)$-complexo com operador bordo $d$.

Usando $h$ e $C$ podemos construir um $\left(R^{\prime}, G^{\prime}\right)$-complexo que denotaremos por $C_{h}$ da seguinte maneira: para cada $i$, seja $\left\{c_{1}^{i}, \ldots, c_{n_{i}}^{i}\right\}$ uma base distinguida para $C_{i}$. Defina $\left(C_{h}\right)_{i}$ como sendo o $R^{\prime}$-módulo com base $\left\{c_{1}^{i}, \ldots, c_{n_{i}}^{i}\right\}$, ou seja, os elementos de $\left(C_{h}\right)_{i}$ são somas formais $\sum_{j} r_{j}^{\prime} c_{j}^{i} \operatorname{com} r_{j}^{\prime} \in R^{\prime}$ e as operações de $\left(C_{h}\right)_{i}$ são dadas por

$$
\sum_{j} r_{j}^{\prime} c_{j}^{i}+\sum_{j} \rho_{j}^{\prime} c_{j}^{i}=\sum_{j}\left(r_{j}^{\prime}+\rho_{j}^{\prime}\right) c_{j}^{i}
$$

$\mathrm{e}$

$$
\rho^{\prime}\left(\sum_{j} r_{j}^{\prime} c_{j}^{i}\right)=\sum_{j}\left(\rho^{\prime} r_{j}^{\prime}\right) c_{j}^{i}
$$

O operador bordo $d_{h}$ de $C_{h}$ é definido como $\left\langle\left(d_{h}\right)_{i}\right\rangle=h_{*}\left(\left\langle d_{i}\right\rangle\right)$, onde $h_{*}$ : $G L(R) \rightarrow G L\left(R^{\prime}\right)$ é a aplicação que associa a uma matriz $\left(a_{j k}\right) \in G L(R)$ a matriz $\left(h\left(a_{j k}\right)\right) \in G L\left(R^{\prime}\right)$. Claramente, $\left(d_{h}\right)_{i}\left(d_{h}\right)_{i+1}=0$. Por convenção, as bases $\left\{c_{1}^{i}, \ldots, c_{n_{i}}^{i}\right\}$ são as bases distinguidas para os $R^{\prime}$-módulos $\left(C_{h}\right)_{i}$. Assim, $C_{h}$ é um $\left(R^{\prime}, G^{\prime}\right)$-complexo.

Mostra-se sem dificuldades que o complexo $C_{h}$ independe, a menos de isomorfismo simples, da escolha da base distinguida para $C$. Assim, obtemos um invariante algébrico para $C$ dado por

$$
\tau_{h}(C)=\tau\left(C_{h}\right) \in K_{G^{\prime}}\left(R^{\prime}\right) .
$$

Proposição 3.26. Sejam $C$ um $(R, G)$-complexo acíclico e $h: R \rightarrow R^{\prime}$ um homomorfismo de anéis com $h(G) \subset G^{\prime}$. Então o $\left(R^{\prime}, G^{\prime}\right)$-complexo $C_{h}$ é também acíclico e vale $\tau_{h}(C)=h_{*} \tau(C)$ onde $h_{*}: K_{G}(R) \rightarrow K_{G^{\prime}}\left(R^{\prime}\right)$ é a aplicação induzida de $h$.

Demonstração. Inicialmente, observe que se $C^{\prime}$ é um complexo de cadeia livre sobre $R$ que tem contração de cadeia $\delta^{\prime}$, então $C^{\prime}$ é acíclico. De fato, para cada $i$, temos que $d_{i+1}^{\prime} C_{i+1}^{\prime} \subset \operatorname{Ker}\left(d_{i}^{\prime}\right)$, pois $d_{i}^{\prime} d_{i+1}^{\prime}=0$. Por outro lado, se $x \in C_{i}^{\prime}$ é tal que $d_{i}^{\prime}(x)=0$, então $x=\left(\delta_{i}^{\prime} d_{i}^{\prime}+d_{i+1}^{\prime} \delta i+1^{\prime}\right)(x)=d_{i+1}^{\prime}\left(\delta_{i+1}^{\prime}(x)\right) \in d_{i+1}^{\prime} C_{i+1}^{\prime}$, donde $\operatorname{Ker}\left(d_{i}^{\prime}\right) \subset d_{i+1}^{\prime} C_{i+1}^{\prime}$. 
Seja $\delta$ uma contração de cadeia para $C$. Suponha que $\left\langle\delta_{i}\right\rangle=\left(b_{j k}\right)$, então defina $\left(\delta_{h}\right)_{i}:\left(C_{h}\right)_{i-1} \rightarrow\left(C_{h}\right)_{i}$ como a aplicação que tem como matriz $\left\langle\left(\delta_{h}\right)_{i}\right\rangle=\left(h\left(b_{j k}\right)\right)$. Procedendo de maneira idêntica para cada $i$, obtemos uma aplicação $\delta_{h}: C_{h} \rightarrow C_{h}$ de grau um. Mostra-se com facilidade que $\delta_{h}$ é uma contração de cadeia para $C_{h}$.

Claramente, $h_{*}(\langle d+\delta\rangle)=\left\langle d_{h}+\delta_{h}\right\rangle$, donde $\tau_{h}(C)=\tau\left(C_{h}\right)=h_{*} \tau(C)$.

Corolário 3.27. Seja $C$ um $W h(G)$-complexo acíclico com operador bordo d. Suponha que existe uma base distinguida para cada $C_{i}$ de maneira que a matriz de $d_{i}$ com respeito a estas bases é da forma $\left(a_{j k}\right)$ com cada $a_{j k} \in \mathbb{Z} \subset$ $\mathbb{Z} G$. Então $\tau(C)=0$.

Demonstração. Considere a aplicação $A: \mathbb{Z} G \rightarrow G$ dada por

$$
A\left(\sum_{i} n_{i} g_{i}\right)=\sum_{i} n_{i}
$$

Trata-se de um homomorfismo de anéis tal que $A(T) \subset\{-1,1\}$.

Denote o $(\mathbb{Z},\{-1,1\})$-complexo acíclico $C_{A}$ por $C^{\prime}$. Observe que $\tau\left(C^{\prime}\right)=$ 0 , pois pela demostração de 2.23 $W h(\{1\})=0$.

Seja $h:(\mathbb{Z},\{-1,1\}) \rightarrow(\mathbb{Z} G, T)$ a aplicação inclusão.

Considere agora o $W h(G)$-complexo acíclico $C_{h}^{\prime}$. Claramente $C \cong_{S} C_{h}^{\prime}$.

Então, temos: $\tau(C)=\tau\left(C_{h}^{\prime}\right)=h_{*} \tau\left(C^{\prime}\right)=h_{*}(0)=0 \in W h(G)$. 


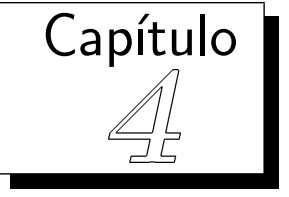

\section{Torção de Whitehead de um par CW}

\subsection{Definição de torção de Whitehead de um par CW}

Seja $(K, L)$ um par de complexos CW conexos e finitos tais que $K{ }_{\downarrow} L$. Temos que as seguintes afirmações são válidas:

(a) Como $K$ é um complexo $\mathrm{CW}$ conexo, $K$ possui um recobrimento universal. Denotaremos este recobrimento por $p: \tilde{K} \rightarrow K$.

(b) $\tilde{L}=p^{-1}(L)$ é um espaço de recobrimento universal de $L$. Além disso, $\tilde{K} \leadsto \tilde{L}$.

(c) O complexo de cadeia celular $C(\tilde{K}, \tilde{L})$ é um $\mathbb{Z} G$-complexo, onde $G=$ $\operatorname{Cov}(\tilde{K})$.

(d) Se, para cada célula $e_{\alpha} \in K-L$, fixarmos uma aplicação característica $\varphi_{\alpha}$ e um levantamento $\tilde{\varphi}_{\alpha}$ de $\varphi_{\alpha}$, então $B=\left\{\left\langle\tilde{\varphi}_{\alpha}\right\rangle \mid e_{\alpha} \in K-L\right\}$ é uma base para o $\mathbb{Z} G$-complexo $C(\tilde{K}, \tilde{L})$.

Denote por $\mathcal{B}$ o conjunto de todas as bases obtidas de maneira análoga a da afirmação acima. 
Proposição 4.1. O complexo $C(\tilde{K}, \tilde{L})$ com a família $\mathfrak{B}$ forma um $W h(G)$ complexo acíclico.

Demonstração. Temos que $H(C(\tilde{K}, \tilde{L})) \cong H(|\tilde{K}|,|\tilde{L}|)$.

Como a inclusão $i: \tilde{L} \rightarrow \tilde{K}$ é uma equivalência de homotopia simples, pois $\tilde{K} \smile_{\downarrow} \tilde{L}$, temos que a aplicação induzida $i_{*}: H_{n}(|\tilde{L}|) \rightarrow H_{n}(|\tilde{K}|)$ é um isomorfismo de grupos. Agora, temos que a seqüência exata do par $(|\tilde{K}|,|\tilde{L}|)$ contém

$\cdots \rightarrow H_{n}(|\tilde{L}|) \stackrel{\cong}{\longrightarrow} H_{n}(|\tilde{K}|) \rightarrow H_{n}(|\tilde{K}|,|\tilde{L}|) \rightarrow H_{n-1}(|\tilde{L}|) \stackrel{\cong}{\longrightarrow} H_{n-1}(|\tilde{K}|) \rightarrow \ldots$ donde $H_{n}(|\tilde{K}|,|\tilde{L}|)=0, \forall n$.

Sejam $c, c^{\prime} \in \mathcal{B}$ bases que se restringem a bases $c_{n}=\left\{\left\langle\tilde{\varphi}_{1}\right\rangle, \ldots,\left\langle\tilde{\varphi}_{q}\right\rangle\right\} \mathrm{e}$ $c_{n}^{\prime}=\left\{\left\langle\tilde{\psi}_{1}\right\rangle, \ldots,\left\langle\tilde{\psi}_{q}\right\rangle\right\}$ para $C_{n}(\tilde{K}, \tilde{L})$.

Então

$$
\left\langle\tilde{\psi}_{j}\right\rangle=\sum_{k} a_{j k}\left\langle\tilde{\varphi}_{k}\right\rangle
$$

onde $a_{j k}$ é igual a soma finita $\sum_{i} n_{i}^{j k} g_{i} \in \mathbb{Z} G, \operatorname{com} n_{i}^{j k} \in \mathbb{Z}$ e $g_{i} \in G$.

Assim,

$$
\begin{aligned}
\left\langle\tilde{\psi}_{j}\right\rangle & =\sum_{k}\left(\sum_{i} n_{i}^{j k} g_{i}\right)\left\langle\tilde{\varphi}_{k}\right\rangle \\
& =\sum_{k} \sum_{i} n_{i}^{j k}\left\langle g_{i} \tilde{\varphi}_{k}\right\rangle \\
& =\sum_{k, i} n_{i}^{k j}\left\langle g_{i} \tilde{\varphi}_{k}\right\rangle .
\end{aligned}
$$

Mas a célula $\tilde{\psi}_{j}\left(\stackrel{\circ}{ }^{n}\right)$, como um levantamento de $e_{j}$, é igual a uma das células $g_{i_{j}} \tilde{\varphi}_{j}\left(\stackrel{\circ}{ }^{n}\right)$ e é disjunta de todas as outras. Então, pelo resultado 1.9, temos que os coeficientes $n_{i}^{j k}$ são nulos exceto para algum $N=n_{i_{j}}^{j j}$.

Por outro lado, $\tilde{\varphi}_{j}\left(\stackrel{\circ}{I^{n}}\right)=g_{i_{j}}^{-1} \tilde{\psi}_{j}\left(\stackrel{\circ}{I^{n}}\right)$, logo $\left\langle\tilde{\varphi}_{j}\right\rangle=N^{\prime}\left\langle g_{i_{j}}^{-1} \tilde{\psi}_{j}\right\rangle$.

Assim, $\left\langle\tilde{\psi}_{j}\right\rangle=N\left\langle g_{i_{j}} \tilde{\varphi}_{j}\right\rangle=N g_{i_{j}}\left\langle\tilde{\varphi}_{j}\right\rangle=N g_{i_{j}}\left(N^{\prime}\left\langle g_{i_{j}}^{-1} \tilde{\psi}_{j}\right\rangle\right)=$ $N g_{i_{j}}\left(N^{\prime} g_{i_{j}}^{-1}\left\langle\tilde{\psi}_{j}\right\rangle\right)=N N^{\prime}\left\langle\tilde{\psi}_{j}\right\rangle$. 
Segue que $N= \pm 1$, donde $\left\langle\tilde{\psi}_{j}\right\rangle= \pm g_{i_{j}}\left\langle\tilde{\varphi}_{j}\right\rangle$.

Logo,

$$
\left\langle c_{n}^{\prime} / c_{n}\right\rangle=\left(\begin{array}{cccc} 
\pm g_{i_{1}} & 0 & \ldots \ldots \ldots \\
0 & \pm g_{i_{2}} & 0 & \ldots \\
\vdots & & \ddots & \\
0 & \ldots \ldots & \ldots & \pm g_{i_{q}}
\end{array}\right)
$$

Segue que $\tau\left(\left\langle c_{n}^{\prime} / c n\right\rangle\right)=0 \in W h(G)$.

Portanto, $C(\tilde{K}, \tilde{L})$ torna-se um $W h(G)$-complexo se estipularmos que $b$ é uma base distinguida se e somente se $\tau(\langle c / b\rangle)=0$.

Neste momento cabe recordar que existe um funtor covariante da categoria dos grupos e homomorfismo de grupos nela mesmo que leva cada grupo $G$ a seu grupo de Whitehead $W h(G)$ e cada homomorfismo $G_{1} \rightarrow G_{2}$ num homomorfismo induzido de forma natural $W h\left(G_{1}\right) \rightarrow W h\left(G_{2}\right)$.

Seja $X$ um espaço conexo por caminhos. Tome $x, y \in X$ e um caminho $\alpha:(I, 0,1) \rightarrow(X, x, y)$. Este caminho induz um isomorfismo de grupos $f_{\alpha}: \pi_{1}(X, x) \rightarrow \pi_{1}(X, y)$ dado por $f_{\alpha}[\omega]=[\bar{\alpha} * \omega * \alpha]$.

O funtor comentado anteriormente leva este isomorfismo a um isomorfismo, $\left(f_{\alpha}\right)_{*}: W h\left(\pi_{1}(X, x)\right) \rightarrow W h\left(\pi_{1}(X, y)\right)$.

Proposição 4.2. Nas condições acima, temos que $\left(f_{\alpha}\right)_{*}$ independe do caminho $\alpha$ escolhido, ou seja, se $\beta$ é um outro caminho em $X$ tal que $\beta(0)=x$ e $\beta(1)=y$, então $\left(f_{\alpha}\right)_{*}=\left(f_{\beta}\right)_{*}$. Assim, denotaremos $\left(f_{\alpha}\right)_{*}=f_{x, y}$. Seja z um outro elemento de $X$, então vale $f_{y, z} \circ f_{x, y}=f_{x, z}$ (Referência: [5])

Teorema 4.3. Sejam $\tilde{p}: \tilde{K} \rightarrow K$ e $\hat{p}: \hat{K} \rightarrow K$ recobrimentos universais do complexo $C W$ conexo $K, \tilde{G}=\operatorname{Cov}(\tilde{K})$ e $\hat{G}=\operatorname{Cov}(\hat{K}), x, y \in K, \tilde{x}=\tilde{p}^{-1}(x) e$ $\hat{y}=\hat{p}^{-1}(y), \tilde{\psi}: \tilde{G} \rightarrow \pi_{1}(K, x)$ e $\hat{\psi}: \hat{G} \rightarrow \pi_{1}(K, y)$ os isomorfismos de grupos determinados por $(x, \tilde{x}) e(y, \hat{y})$. Então $\tau\left(C(\hat{K}, \hat{L})_{\hat{\psi}}\right)=f_{x, y} \tau\left(C(\tilde{K}, \tilde{L})_{\tilde{\psi}}\right)$.

Demonstração. Seja $h: \tilde{K} \rightarrow \hat{K}$ um levantamento de $\tilde{p}$. Então $h$ é um homeomorfismo, logo, por (1.12), é um isomorfismo celular. 
Defina $H: \tilde{G} \rightarrow \hat{G}$ por $H(g)=h g h^{-1}$.

Afirmamos que $\tau(C(\hat{K}, \hat{L}))=H_{*} \tau(C(\tilde{K}, \tilde{L}))$, onde $H_{*}: W h(\tilde{G}) \rightarrow$ $W h(\hat{G})$ é a aplicação induzida de $H$.

De fato, seja $\left\{\left\langle\tilde{\varphi}_{k}^{i}\right\rangle\right\}$ uma base para $C_{i}(\tilde{K}, \tilde{L})$ como em 1.12 , e seja $\hat{\varphi}_{k}^{i}=h \tilde{\varphi}_{k}^{i}, \forall i, k$. Claramente $\left\{\left\langle\hat{\varphi}_{k}^{i}\right\rangle\right\}$ é uma base para $C_{i}(\hat{K}, \hat{L})$ como em (1.12).

Agora, $h$ induz um isomorfismo de cadeia $h_{*}: C(\tilde{K}, \tilde{L}) \rightarrow C(\hat{K}, \hat{L})$ quando estes complexos são pensados como complexos sobre $\mathbb{Z}$.

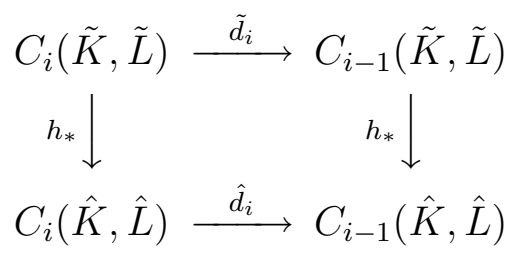

Temos então que $\hat{d}_{i}=h_{*} \tilde{d}_{i} h_{*}^{-1}$.

Seja $\left(a_{k j}\right) \in G L(\mathbb{Z} \tilde{G})$ a matriz do operador bordo $\tilde{d}_{i}$.

Então, veja que

$$
\begin{aligned}
\hat{d}_{i}\left(\left\langle\hat{\varphi}_{k}^{i}\right\rangle\right) & =h_{*} \tilde{d}_{i} h_{*}^{-1}\left(\left\langle\hat{\varphi}_{k}^{i}\right\rangle\right) \\
& =h_{*} \tilde{d}_{i}\left(\left\langle h^{-1} \hat{\varphi}_{k}^{i}\right\rangle\right) \\
& =h_{*} \tilde{d}_{i}\left(\left\langle\tilde{\varphi}_{k}^{i}\right\rangle\right) \\
& =h_{*}\left(\sum_{j} a_{k j}\left\langle\tilde{\varphi}_{j}^{i-1}\right\rangle\right) \\
& =\sum_{j} h_{*}\left(a_{k j}\left\langle\tilde{\varphi}_{j}^{i-1}\right\rangle\right) .
\end{aligned}
$$

Suponha que $a_{k j}=\sum_{l} n_{l}^{k j} g_{l}^{k j}$, onde $n_{l}^{k j} \in \mathbb{Z}$ e $g_{l}^{k j} \in \tilde{G}$, então $a_{k j}\left\langle\tilde{\varphi}_{j}^{i-1}\right\rangle=$ $\left(\sum_{l} n_{l}^{k j} g_{l}^{k j}\right)\left\langle\tilde{\varphi}_{j}^{i-1}\right\rangle=\sum_{l} n_{l}^{k j}\left\langle g_{l}^{k j} \tilde{\varphi}_{j}^{i-1}\right\rangle$. 
Assim,

$$
\begin{aligned}
\hat{d}_{i}\left(\left\langle\hat{\varphi}_{k}^{i}\right\rangle\right) & =\sum_{j} h_{*}\left(\sum_{l} n_{l}^{k j}\left\langle g_{l}^{k j} \tilde{\varphi}_{j}^{i-1}\right\rangle\right) \\
& =\sum_{j} \sum_{l} n_{l}^{k j} h_{*}\left(\left\langle g_{l}^{k j} \tilde{\varphi}_{j}^{i-1}\right\rangle\right) \\
& =\sum_{j} \sum_{l} n_{l}^{k j}\left\langle h g_{l}^{k j} \tilde{\varphi}_{j}^{i-1}\right\rangle \\
& =\sum_{j} \sum_{l} n_{l}^{k j}\left\langle h g_{l}^{k j} h^{-1} \hat{\varphi}_{j}^{i-1}\right\rangle \\
& =\sum_{j} \sum_{l} n_{l}^{k j}\left\langle H\left(g_{l}^{k j}\right) \hat{\varphi}_{j}^{i-1}\right\rangle \\
& =\sum_{j}\left(\sum_{l} n_{l}^{k j} H\left(g_{l}^{k j}\right)\right)\left\langle\hat{\varphi}_{j}^{i-1}\right\rangle \\
& =\sum_{j} H_{*}\left(a_{k j}\right)\left\langle\hat{\varphi}_{j}^{i-1}\right\rangle,
\end{aligned}
$$

onde $H_{*}: \mathbb{Z} \tilde{G} \rightarrow \mathbb{Z} \hat{G}$ é a aplicação induzida de $H$.

Logo, a matriz do operador bordo $\hat{d}_{i}$ é dada por $H_{*}\left(\left(a_{k j}\right)\right)$.

Segue, da demonstração do resultado 3.26 , que $\tau(C(\hat{K}, \hat{L}))=$ $H_{*} \tau(C(\tilde{K}, \tilde{L}))$.

Agora, sejam $\hat{x}=h(\tilde{x})$ e $\Omega:(I, 0,1) \rightarrow(\hat{K}, \hat{x}, \hat{y})$ um caminho arbitrário.

Defina $\omega=\hat{p} \Omega$ e seja $f_{\omega}: \pi_{1}(K, x) \rightarrow \pi_{1}(K, y)$ o isomorfismo discutido na proposição 4.2).

Então, denotando $\tilde{\theta}=\theta(x, \tilde{x})=\tilde{\psi}^{-1}$ e $\hat{\theta}=\theta(y, \hat{y})=\hat{\psi}^{-1}$, o seguinte diagrama comuta

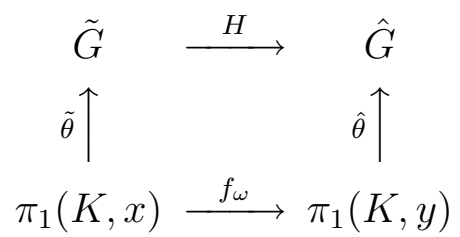


pois, se $[\alpha] \in \pi_{1}(K, x)$, temos

$$
\begin{aligned}
\left(\hat{\theta} f_{\omega}[\alpha]\right)(\hat{y}) & =(\hat{\theta}[\bar{\omega} * \alpha * \omega])(\hat{y}) \\
& =\hat{\theta}_{[\bar{\omega} * \alpha * \omega]}(\hat{y}) \\
& =\widehat{\alpha * \omega}(1), \text { onde } \widehat{\alpha * \omega}(0)=\hat{x} \\
& =h(\widetilde{\alpha * \omega}(1)) \\
& =h\left(\tilde{\theta}_{[\alpha]} h^{-1} \Omega(1)\right), \text { pois } \omega=\tilde{p} h^{-1} \Omega \\
& =h\left(\tilde{\theta}_{[\alpha]} h^{-1}(\hat{y})\right) \\
& =\left(h \tilde{\theta}_{[\alpha]} h^{-1}\right)(\hat{y}) \\
& =(H \tilde{\theta}[\alpha])(\hat{y}) .
\end{aligned}
$$

Como $\hat{\theta} f_{\omega}[\alpha]$ e $H \tilde{\theta}[\alpha]$ coincidem em um ponto de $\hat{K}$, estes homeomorfismos coincidem em todo $\hat{K}$, assim $\hat{\theta} f_{\omega}[\alpha]=H \tilde{\theta}[\alpha]$ e, portanto, $\hat{\theta} f_{\omega}=H \tilde{\theta}$.

Temos então que

$$
\begin{aligned}
f_{x, y} \tau\left(C(\tilde{K}, \tilde{L})_{\tilde{\psi}}\right) & =\left(f_{\omega}\right)_{*} \tau\left(C(\tilde{K}, \tilde{L})_{\tilde{\psi}}\right) \\
& =\left(f_{\omega}\right)_{*} \tilde{\psi}_{*} \tau(C(\tilde{K}, \tilde{L})) \\
& =\hat{\psi}_{*} H_{*} \tau(C(\tilde{K}, \tilde{L})) \\
& =\hat{\psi} \tau(C(\hat{K}, \hat{L})) \\
& =\tau\left(C(\hat{K}, \hat{L})_{\hat{\psi}}\right) .
\end{aligned}
$$

Sobre o conjunto $\bigcup_{x \in K} W h\left(\pi_{1}(K, x)\right)$ considere a seguinte relação de equivalência

$$
a \sim b \Longleftrightarrow a \in W h\left(\pi_{1}(K, x)\right), b \in W h\left(\pi_{1}(k, y)\right) \text { e } f_{x, y}(a)=b .
$$

Denote por $W h\left(\pi_{1} K\right)$ o conjunto das classes de equivalência desta relação. Considere a bijeção natural $j_{x}: W h\left(\pi_{1}(K, x)\right) \rightarrow W h\left(\pi_{1} K\right)$. Estipulando que $j_{x}$ é um isomorfismo de grupos, obtemos uma estrutura de grupo para $W h\left(\pi_{1} K\right)$. Esta estrutura de grupo não depende da escolha de $x \in K$. (Isto é provado facilmente, usando apenas que $j_{y}^{-1} j_{x}=f_{x, y}$.) 
Seja $f:(K, x) \rightarrow\left(K^{\prime}, x^{\prime}\right)$ uma aplicação e deixe que $f_{\sharp}$ denote os homomorfismos $\pi_{1}(K, x) \rightarrow \pi_{1}\left(K^{\prime}, x^{\prime}\right)$ e $W h\left(\pi_{1}(K, x)\right) \rightarrow W h\left(\pi_{1}\left(K^{\prime}, x^{\prime}\right)\right)$ induzidos por $f$.

Agora, considere a aplicação composta

$$
f_{*}: W h\left(\pi_{1} K\right) \stackrel{j_{x}^{-1}}{\longrightarrow} W h\left(\pi_{1}(K, x)\right) \stackrel{f_{\sharp}}{\longrightarrow} W h\left(\pi_{1}\left(K^{\prime}, x^{\prime}\right)\right) \stackrel{j_{x^{\prime}}}{\longrightarrow} W h\left(\pi_{1} K^{\prime}\right) .
$$

Proposição 4.4. Nas condições acima descritas, tem-se que o homomorfismo $f_{*}$ independe da escolha do par $\left(x, x^{\prime}\right)$ com $f(x)=x^{\prime}$. (Referência: [5])

Proposição 4.5. Existe um funtor covariante da categoria de complexos $C W$ conexos e finitos e aplicações na categoria de grupos abelianos e homomorfismos de grupos definido por

$$
\begin{gathered}
K \mapsto W h\left(\pi_{1} K\right) \\
\left\{f: K \rightarrow K^{\prime}\right\} \mapsto\left\{f_{*}: W h\left(\pi_{1} K\right) \rightarrow W h\left(\pi_{1} K^{\prime}\right)\right\} .
\end{gathered}
$$

Além disso, se $f \simeq g$ então $f_{*}=g_{*}$.

Dado um par homotopicamente trivial de complexos CW conexos e finitos $(K, L)$, escolha um $x \in K$, um recobrimento universal $p:(\tilde{K}, \tilde{L}) \rightarrow(K, L)$ e um $\tilde{x} \in p^{-1}(x)$. Seja $i: L \rightarrow K$ a aplicação inclusão. Observe que, como $K{ }^{2} L, i_{*}$ é um isomorfismo.

Definição 4.1. Nas condições acima, definimos a torção do par $(K, L)$ e a denotamos por $\tau(K, L)$ o elemento de $W h\left(\pi_{1} L\right)$ do final da seqüência

$$
\begin{gathered}
\tau(C(\tilde{K}, \tilde{L})) \in W h(G) \\
\downarrow \tilde{\psi}(x, \tilde{x})_{*} \\
\tau\left(C(\tilde{K}, \tilde{L})_{\tilde{\psi}}\right) \in W h\left(\pi_{1}(K, x)\right) \\
\downarrow^{j_{x}} \\
\tau^{\prime} \in W h\left(\pi_{1} K\right) \\
\downarrow^{-1} \\
\tau(K, L) \in W h\left(\pi_{1} L\right)
\end{gathered}
$$


Esta definição pode ser estendida para o caso não-conexo da seguinte forma: seja $(K, L)$ um par homotopicamente trivial de complexos CW conexos; sejam $L_{1}, \ldots, L_{q}$ as componentes de L (existem finitas componentes para $L$ pois $L$ é um complexo CW finito); então $K$ tem também $q$ componentes $K_{1}, \ldots, K_{q}$ (pois $K{ }_{\downarrow} L$ ) que podem ser ordenadas de maneira que $K_{j} \aleph_{\downarrow} L_{j}, \forall j$. Então definimos

$$
\tau(K, L)=\sum_{j} \tau\left(K_{j}, L_{j}\right) \in \oplus W h\left(\pi_{1} L_{j}\right) .
$$

Proposição 4.6. Existe um funtor covariante da categoria de complexos CW finitos e aplicações na categoria de grupos abelianos e homomorfismos de grupos definido por

$$
\begin{aligned}
& K \mapsto \oplus_{j} W h\left(\pi_{1} K_{j}\right) \quad\left(K_{1}, \ldots, K_{q} \text { são as componentes de } K\right) \\
& \left\{f: K \rightarrow K^{\prime}\right\} \mapsto\left\{f_{*}=\sum_{j}\left(f_{j}\right)_{*}: \oplus_{j} W h\left(\pi_{1} K_{j}\right) \rightarrow \oplus_{i} W h\left(\pi_{1} K_{i}^{\prime}\right)\right\}
\end{aligned}
$$

onde $\left(f_{j}\right)_{*}: W h\left(\pi_{1} K_{j}\right) \rightarrow W h\left(\pi_{1} K_{i_{j}}^{\prime}\right)$ é induzida de $f$ com $f\left(K_{j}\right) \subset K_{i_{j}}^{\prime}$. Além disso, se $f \simeq g$, então $f_{*}=g_{*}$.

\subsection{Propriedades fundamentais da torção de um par}

Nesta seção apresentaremos resultados fundamentais cujas demonstrações são técnicas e não acrescentam informações úteis ao desenvolvimento deste trabalho, por isso serão omitidas. Contudo as provas destes resultados podem ser encontradas em [5].

Proposição 4.7. Seja $(K, L)$ um par homotopicamente trivial de complexos $C W$ finitos. Suponha que cada componente de $K-L$ é simplesmente conexa. Então $\tau(K, L)=0$.

Proposição 4.8. Sejam $K, L$ e $M$ complexos $C W$ finitos tais que $M<L<$ $K, K \overbrace{\downarrow} L$ e $L$ \ $M$. Então

$$
\tau(K, M)=\tau(L, M)+i_{*}^{-1} \tau(K, L),
$$

onde $i: M \rightarrow L$ é a aplicação inclusão. 
Lema 4.9 (Lema da Excisão). Sejam $K, L$ e $M$ subcomplexos do complexo $C W$ finito $K \cup L, \operatorname{com} M=K \cap L$ e $K{ }_{\downarrow} M$. Então $\tau(K \cup L, L)=$ $j_{*} \tau(K, M)$, onde $j: M \rightarrow L$ é a aplicação inclusão.

Corolário 4.10. Sejam $K, L$ e $M$ subcomplexos do complexo $C W$ finito $K \cup$ $L, \operatorname{com} M=K \cap L, K{ }_{\downarrow} M$ e $L{ }_{\downarrow} M$. Então $\tau(K \cup L, M)=\tau(K, M)+$ $\tau(L, M)$.

Proposição 4.11. Seja $(K, L)$ um par de complexos $C W$ conexos e finitos na forma simplificada $K=L \cup \bigcup_{j} e_{j}^{n} \cup \bigcup_{i} e_{i}^{n+1}$, com $n \geq 2$ e aplicações características $\left\{\varphi_{j}\right\}$ e $\left\{\psi_{i}\right\}$ para as células $e_{j}^{n}$ e $e_{i}^{n+1}$, respectivamente. Denote $K_{n}=L \cup \bigcup_{j} e_{j}^{n}$. Seja $\langle\partial\rangle$ a matriz, com entradas em $\mathbb{Z}\left(\pi_{1}\left(L, e^{0}\right)\right)$, do operador bordo $\partial: \pi_{n+1}^{j}\left(K, K_{n} ; e^{0}\right) \rightarrow \pi_{n}\left(K_{n}, L ; e^{0}\right)$, apresentado na seção 2.5), com relação às bases $\left\{\left[\varphi_{j}\right]\right\}$ e $\left\{\left[\psi_{i}\right]\right\}$. Então $\tau(K, L)=(-1)^{n} \tau(\langle\partial\rangle)$.

\subsection{A equivalência natural entre $W h(L)$ e $\oplus W h\left(\pi_{1} L_{j}\right)$}

Nas seções anteriores definimos dois funtores da categoria dos complexos CW finitos e aplicações na categoria dos grupos abelianos e homomorfismos de grupos. O primeiro, definido na seção 2.3 , é dado por

$$
\begin{gathered}
L \mapsto W h(L) \\
\left\{f: L \rightarrow L^{\prime}\right\} \mapsto\left\{f_{*}: W h(L) \rightarrow W h\left(L^{\prime}\right)\right\}
\end{gathered}
$$

e o segundo, definido na seção 4.1), é dado por

$$
\begin{gathered}
L \mapsto \oplus_{j} W h\left(\pi_{1} L_{j}\right) \quad\left(L_{1}, \ldots, L_{q} \text { são as componentes de } L\right) \\
\left\{f: L \rightarrow L^{\prime}\right\} \mapsto\left\{f_{*}=\sum_{j}\left(f_{j}\right)_{*}: \oplus_{j} W h\left(\pi_{1} L_{j}\right) \rightarrow \oplus_{i} W h\left(\pi_{1} L_{i}^{\prime}\right)\right\} .
\end{gathered}
$$

Mostremos que existe uma equivalência natural entre estes dois funtores.

Teorema 4.12. Para cada complexo $C W$ finito $L$ com componentes $L_{1}, \ldots, L_{q}$, considere a aplicação

$$
T_{L}: W h(L) \rightarrow \oplus_{j} W h\left(\pi_{1} L_{j}\right)
$$




$$
T_{L}([K, L])=\tau(K, L)
$$

Então $T=\left\{T_{L}\right\}$ é uma equivalência natural de funtores.

Demonstração. Sejam $K$ e $K^{\prime}$ complexos finitos que têm $L$ como subcomplexo e que satisfazem $K \backslash L$ e $K^{\prime} \downarrow L$. Suponha que $K^{\prime} \searrow^{e} K$, então $K^{\prime}{ }_{\downarrow} K$ e $K^{\prime}-K$ é simplesmente conexo. Segue, de (4.8), que $\tau\left(K^{\prime}, L\right)=\tau(K, L)+i_{*}^{-1} \tau\left(K^{\prime}, K\right)$, onde $i: L \rightarrow K$ é a inclusão. Mas, por (4.7), $\tau\left(K^{\prime}, K\right)=0$, donde $\tau\left(K^{\prime}, L\right)=\tau(K, L)$.

Por indução sobre o número de colapsos e expansões elementares obtemos que $\tau\left(K^{\prime}, L\right)=\tau(K, L)$ sempre que $K \wedge K^{\prime}$ rel $L$.

Logo, para cada $L, T_{L}$ está bem definida.

A prova de que $T_{L}$ é um homomorfismo é imediata de (4.10).

Agora, suponha que $T_{L}([K, L])=\tau(K, L)=0$ e que $L$ é conexo. Então, por (2.18), existe um complexo $M$ tal que $M \wedge K$ rel $L$ e $(M, L)$ é um par na forma simplificada.

Temos que $[M, L]=[K, L], \log \sigma(K, L)=\tau(M, L)$.

Por 4.11), temos que $\tau(M, L)=(-1)^{n} \tau(\langle\partial\rangle)$, onde $\langle\partial\rangle$ é a matriz do par $(M, L)$. Então, $\tau(\langle\partial\rangle)=0$.

Segue, de (2.20), que $M \wedge L$ rel $L$, donde $K \wedge L$ rel $L$, e, portanto, $[K, L]=0$.

A prova de que $T_{L}([K, L])=0 \Rightarrow[K, L]=0$ para o caso em que $L$ não é conexo e apenas uma generalização do que fizemos acima.

Logo, $T_{L}$ é um monomorfismo.

Os resultados (4.11) e (2.25), garantem que $T_{L}$ é uma aplicação sobrejetiva.

Assim, para cada $L, T_{L}$ é um isomorfismo.

Mostremos, por fim, que se $f: L \rightarrow L^{\prime}$ é uma aplicação arbitrária, então 
o seguinte diagrama comuta

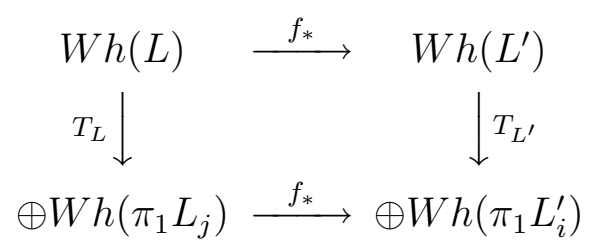

Podemos assumir que $f$ é uma aplicação celular. Assim, dado $[K, L] \in$ $W h(L)$, temos $T_{L^{\prime}} f_{*}[K, L]=T_{L^{\prime}}\left[K \cup_{L} M_{f}, L^{\prime}\right]=\tau\left(K \cup_{L} M_{f}, L^{\prime}\right)=$ $\tau\left(M_{f}, L^{\prime}\right)+i_{*}^{-1} \tau\left(K \cup_{L} M_{f}, M_{f}\right)$, onde $i: L^{\prime} \rightarrow M_{f}$ é a aplicação inclusão.

Agora, $\tau\left(M_{f}, L^{\prime}\right)=0$, pois $M_{f} \searrow L$. Logo, $T_{L^{\prime}} f_{*}[K, L]=i_{*}^{-1} \tau\left(K \cup_{L}\right.$ $\left.M_{f}, M_{f}\right)$.

Seja $p: M_{f} \rightarrow L^{\prime}$ a projeção natural. Temos que $i_{*}^{-1}=p_{*}$. Segue que $T_{L^{\prime}} f_{*}[K, L]=p_{*} \tau\left(K \cup_{L} M_{f}, M_{f}\right)$.

O lema da excisão, nos fornece que $\tau\left(K \cup_{L} M_{f}, M_{f}\right)=j_{*} \tau(K, L)$, onde $j: L \rightarrow M_{f}$ é a inclusão.

Logo, $T_{L^{\prime}} f_{*}[K, L]=p_{*} j_{*} \tau(K, L)=f_{*} \tau(K, L)=f_{*} T_{L}([K, L])$.

A partir de agora não faremos distinção entre $W h(L)$ e $\oplus_{j} W h\left(\pi_{1} L_{j}\right)$.

\subsection{A torção de uma equivalência de homotopia}

Sejam $K$ e $L$ complexos CW finitos e $f: K \rightarrow L$ uma equivalência de homotopia celular. Então, o resultado 1.8 nos garante que $M_{f}{ }_{\downarrow} K$. Além disso, temos também que $f_{*}: W h(K) \rightarrow W h(L)$ é um isomorfismo de grupos.

Definição 4.2. Tendo em vista as considerações acima, definimos a torção de uma equivalência de homotopia celular $f: K \rightarrow L$ entre complexos CW finitos como sendo o elemento

$$
\tau(f)=f_{*} \tau\left(M_{f}, K\right) \in W h(L) .
$$


As demonstrações dos teoremas a seguir são imediatas, exceto para os teoremas da soma e do produto, cujas demonstrações são encontradas em [5].

Proposição 4.13. Sejam $f, g: K \rightarrow L$ equivalências de homotopia celulares entre complexos $C W$ finitos. Se $f$ e $g$ são aplicações homotópicas, então $\tau(f)=\tau(g)$.

Proposição 4.14. Sejam $K$ e $L$ complexos $C W$ finitos. Uma equivalência de homotopia celular $f: K \rightarrow L$ é uma equivalência de homotopia simples se e somente se $\tau(f)=0$.

Proposição 4.15. Seja $(K, L)$ um par homotopicamente trivial de complexos $C W$ finitos. Então $\tau(i)=i_{*} \tau(K, L)$, onde $i: L \rightarrow K$ é a aplicação inclusão.

Proposição 4.16. Sejam $f: K \rightarrow L$ e $g: L \rightarrow M$ equivalências de homotopia celulares entre complexos $C W$ finitos. Então $\tau(g f)=\tau(g)+g_{*} \tau(f)$.

Corolário 4.17. Sejam $f: K \rightarrow L$ e $g: L \rightarrow K$ equivalências de homotopia celulares entre complexos $C W$ finitos que são homotopicamente inversas entre si. Então $\tau(g)=-g_{*} \tau(f)$.

Teorema 4.18 (Teorema da soma). Sejam $K=K_{1} \cup K_{2}, K_{0}=K_{1} \cap$ $K_{2}, L=L_{1} \cup L_{2}$ e $L_{0}=L_{1} \cap L_{2}$ complexos $C W$ finitos com $K_{0}, K_{1}, K_{2}<$ $K$ e $L_{0}, L_{1}, L_{2}<L$. Sejam $f: K \rightarrow L$ uma aplicação que se restringe a equivalências de homotopia $f_{\alpha}: K_{\alpha} \rightarrow L_{\alpha}, \alpha=0,1,2$ e $j_{\alpha}: L_{\alpha} \rightarrow L$ e $i_{\alpha}: K_{\alpha} \rightarrow K$ inclusões. Então f é uma equivalência de homotopia e

(i) $\tau(f)=\left(j_{1}\right)_{*} \tau\left(f_{1}\right)+\left(j_{2}\right)_{*} \tau\left(f_{2}\right)-\left(j_{0}\right)_{*} \tau\left(f_{0}\right)$

(ii) No caso de $f$ ser uma aplicação inclusão, $\tau(L, K)=\left(i_{1}\right)_{*} \tau\left(L_{1}, K_{1}\right)+$ $\left(i_{2}\right)_{*} \tau\left(L_{2}, K_{2}\right)-\left(i_{0}\right)_{*} \tau\left(L_{0}, K_{0}\right)$.

(Referência: [5])

Teorema 4.19 (Teorema do produto). As seguintes afirmações são válidas 
(i) Sejam $P, K$ e $K_{0}$ complexos $C W$ finitos tais que $K{ }_{\downarrow} K_{0}$ e $P$ é conexo, então

$$
\tau\left(K \times P, K_{0} \times P\right)=\chi(P) \cdot i_{*} \tau\left(K, K_{0}\right),
$$

onde $i: K_{0} \rightarrow K_{0} \times P$ é dada por $i(x)=(x, y)$, para algum $y \in P$ fixado, e $\chi(P)$ denota a característica de Euler de $P$.

(ii) Sejam $f: K \rightarrow L$ e $g: K^{\prime} \rightarrow L^{\prime}$ equivalências de homotopia entre complexos $C W$ conexos e finitos e sejam $i: L \rightarrow L \times L^{\prime}$ e $j: L^{\prime} \rightarrow L \times L^{\prime}$ como no item anterior. Então

$$
\tau(f \times g)=\chi\left(L^{\prime}\right) \cdot i_{*} \tau(f)+\chi(L) \cdot j_{*} \tau(g) .
$$

(Referência: [5])

Uma forma de calcular a torção de uma equivalência de homotopia é dada a seguir.

Sejam $f: K \rightarrow L$ uma equivalência de homotopia celular entre complexos CW conexos e finitos, $\tilde{f}: \tilde{K} \rightarrow \tilde{L}$ um levantamento de $f$ a espaços de recobrimento universal, $\tilde{f}_{*}: C(\tilde{K}) \rightarrow C(\tilde{L})$ a aplicação de cadeia induzida de $\tilde{f}, G_{K}=\operatorname{Cov}(\tilde{K}), G_{L}=\operatorname{Cov}(\tilde{L})$. Pelo que vimos nas seções 1.3 e 1.4), $C(\tilde{K})$ e $C(\tilde{L})$ podem ser vistos como $W h\left(G_{K}\right)$ e $W h\left(G_{L}\right)$-complexos com operadores bordo $d$ e $d^{\prime}$, respectivamente. Fixe pontos $x \in K, y \in L$, $\tilde{x} \in \tilde{K}$, e $\tilde{y} \in \tilde{L}$ de maneira que $f(x)=y$ e $\tilde{f}(\tilde{x})=\tilde{y}$. Seja $f_{*}: G_{K} \rightarrow G_{L}$ a aplicação induzida de $f_{*}: \pi_{1}(K, x) \rightarrow \pi_{1}(L, y)$ como na seção (1.4). Denote também por $f_{*}$ as aplicações induzidas $f_{*}: \mathbb{Z} G_{K} \rightarrow \mathbb{Z} G_{L}$ e $f_{*}: G L\left(\mathbb{Z} G_{K}\right) \rightarrow$ $G L\left(\mathbb{Z} G_{L}\right)$.

Teorema 4.20. Nas condições acima, $\tau(f) \in W h\left(G_{L}\right)$ é a torção do $W h\left(G_{L}\right)$-complexo $\mathcal{C}$ definido a seguir

(a) $\mathcal{C}_{n}=\left[C(\tilde{K})_{f_{*}}\right]_{n-1} \oplus C_{n}(\tilde{L})$ 
(b) $\partial_{n}: \mathcal{C}_{n} \rightarrow \mathcal{C}_{n-1}$ tem matriz

$$
\begin{aligned}
& {\left[C(\tilde{K})_{f_{*}}\right]_{n-2} \quad C_{n-1}(\tilde{L})} \\
& {\left[\begin{array}{c}
\left.C(\tilde{K})_{f_{*}}\right]_{n-1} \\
C_{n}(\tilde{L})
\end{array}\left(\begin{array}{cc}
-f_{*}\left\langle d_{n-1}\right\rangle & \left\langle\tilde{f}_{*}\right\rangle \\
0 & \left\langle d_{n}^{\prime}\right\rangle
\end{array}\right)\right.}
\end{aligned}
$$

(Referência: [5])

\subsection{Relação entre homotopia e homotopia simples}

Seja $L$ um complexo CW finito fixado.

Proposição 4.21. Dado $\tau_{0} \in W h(L)$, existem um complexo $C W$ finito $K e$ uma equivalência de homotopia $f: K \rightarrow L$ tais que $\tau(f)=\tau_{0}$.

Demonstração. Seja $K$ um complexo CW tal que $K \longleftarrow L$ e $\tau(K, L)=-\tau_{0}$. (Um complexo como este existe pela definição de $W h(L)$ na seção 2.3.) Seja $f: K \rightarrow L$ uma aplicação tal que $f$ e a inclusão $i: L \rightarrow K$ são inversas homotópicas. Então $\tau(f)=-f_{*} \tau(i)=-f_{*} i_{*} \tau(K, L)=-\tau(K, L)=\tau_{0}$.

Seja $K$ um complexo CW finito homotopicamente equivalente a $L$ (neste caso denotamos $K \simeq L$ ). Defina

$$
S_{K}=\{\tau(f) \mid f: K \rightarrow L \text { equivalência de homotopia }\} \subset W h(L) .
$$

Teorema 4.22. Se $K$ e $K^{\prime}$ são complexos $C W$ finitos tais que $K \simeq L \simeq K^{\prime}$, então as seguintes afirmativas são equivalentes:

(i) $S_{K} \cap S_{K^{\prime}} \neq \emptyset$

(ii) $K$ e $K^{\prime}$ têm o mesmo tipo de homotopia simples

(iii) $S_{K}=S_{K^{\prime}}$.

Então $\mathcal{F}=\left\{S_{K} \mid K \simeq L\right\}$ forma uma partição de $W h(L)$. 
Demonstração. (i) $\Rightarrow$ (ii) Suponha que $S_{K} \cap S_{K^{\prime}} \neq \emptyset$. Então existem equivalências de homotopia $f: K \rightarrow L$ e $g: K^{\prime} \rightarrow L$ tais que $\tau(f)=\tau(g)$. Seja $\bar{g}: L \rightarrow K^{\prime}$ tal que $g$ e $\bar{g}$ são inversas homotópicos. Assim, $\bar{g} f: K \rightarrow K^{\prime}$ satisfaz $\tau(\bar{g} f)=\tau(\bar{g})+\bar{g}_{*} \tau(f)=-\bar{g}_{*} \tau(g)+\bar{g}_{*} \tau(f)=0$, o que mostra que $K$ e $K^{\prime}$ têm o mesmo tipo de homotopia simples.

(ii) $\Rightarrow$ (iii) Suponha que $s: K^{\prime} \rightarrow K$ é uma equivalência de homotopia simples. Se $\tau_{0} \in S_{K}$, escolha $f: K \rightarrow L \operatorname{com} \tau(f)=\tau_{0}$. Então $f s: K^{\prime} \rightarrow L$ satisfaz $\tau(f s)=\tau(f)+f_{*} \tau(s)=\tau(f)=\tau_{0}$, donde $S_{K} \subset S_{K^{\prime}}$. Por simetria $S_{K^{\prime}} \subset S_{K}$.

(iii) $\Rightarrow$ (i) Trivial, pois $S_{K} \neq \emptyset$.

As seguintes notações serão usadas:

$|S|=$ cardinalidade do conjunto $S$

$\nu_{L}=|\mathcal{F}|$, onde $\mathcal{F}$ é a família definida no resultado anterior.

$\mathcal{E}(L)$ é o conjunto das classes de equivalência das autoequivalências de homotopia de $L$, onde a relação considerada é a homotopia entre aplicações.

$W h_{0}(L)=\left\{\tau(f) \mid f_{*}\right.$ é a aplicação identidade em $\left.W h(L)\right\}$.

Proposição 4.23. $\nu_{L} \cdot\left|W h_{0}(L)\right| \leq|W h(L)| \leq \nu_{L} \cdot|\mathcal{E}(L)|$.

Demonstração. Seja $g: K \rightarrow L$ uma equivalência de homotopia fixada, então a correspondência $f \mapsto f g$, onde $f$ é uma auto-equivalência de homotopia de $L$, induz uma bijeção de $\mathcal{E}(L)$ no conjunto $\mathcal{E}(K, L)$ das classes de equivalência das equivalências de homotopia de $K$ em $L$ (onde a relação considerada é a homotopia entre aplicações). Então, por 4.13), $\left|S_{K}\right| \leq|\mathcal{E}(K, L)|=|\mathcal{E}(L)|$. Agora, $|W h(L)|=\left|\cup_{i} S_{K_{i}}\right|=\sum_{i}\left|S_{K_{i}}\right| \leq \sum_{i}|\mathcal{E}(L)|=|\mathcal{F}||\mathcal{E}(L)|=\nu_{L}|\mathcal{E}(L)|$.

Por outro lado, seja $g_{0}: K \rightarrow L$ uma equivalência de homotopia. Para qualquer $f: L \rightarrow L$ que induz a identidade em $W h(L)$, temos $\tau\left(f g_{0}\right)=$ 
$\tau(f)+\tau\left(g_{0}\right) \in S_{K}$. Então $\tau\left(g_{0}\right)+W h_{0}(L) \subset S_{K}$, donde $\left|W h_{0}(L)\right| \leq\left|S_{K}\right|$. Assim, $\nu_{L}\left|W h_{0}(L)\right| \leq|W h(L)|$.

Teorema 4.24. As seguintes afirmações são válidas:

(i) ( Todo complexo $C W$ finito $K$ com $K \simeq L$ é equivalente por homotopia simples a $L) \Longleftrightarrow(W h(L)=\{\tau(f) \mid f \in \mathcal{E}(L)\})$.

(ii) Se Wh $(L)$ é infinito e $\mathcal{E}(L)$ é finito, então $\mathcal{F}$ é uma família infinita.

Demonstração. (i) Ambas as afirmações em (i) são equivalentes a afirmação que $S_{K}=S_{L}$, para todo $K$.

(ii) Segue trivialmente de 4.22 e de 4.23 .

Observação 2. Todo complexo $\mathrm{CW}$ finito e conexo $L$ de dimensão $2 \mathrm{com}$ $\pi_{1} L \cong \mathbb{Z}_{p}, \quad p \neq 1,2,3,4,6$, tem grupo de Whitehead infinito que satisfaz as condições do primeiro item do teorema acima. Assim, estes complexos satisfazem a conjectura II, embora não satisfaçam a conjectura $\mathbf{I}$.

Por sua vez, todo espaço lenticular $L=L\left(p ; q_{1}, q_{2}, \ldots, q_{n}\right)$, com $p \neq$ $1,2,3,4,6$, satisfaz as hipóteses do item (ii), e então estes espaços não satisfazem a conjectura II. 


\section{Referências Bibliográficas}

[1] BAss, H. - Algebraic K-theory, W. A. Benjamin, Inc., New York, 1968.

[2] Bass, H. - K-theory and stable algebra, Publ. de I'Inst. des Hautes Etudes Sci., \#22, 1964.

[3] Bass, H., Heller, A. And Swan, R. - The Whitehead group of a polynomial extension, Publ. de I'Inst. des Hautes Etudes Sci., \#22, 1964.

[4] Chevalley, C. - Fundamental concepts of algebra, Academic Press, New York, 1956.

[5] Cohen, M. M. - A course in simple-homotopy theory, SpringerVerlag, New York, 1973.

[6] Higman, G. - The units of group rings, Proc. London Math Soc., 46(1940), 231-248.

[7] Hu, S. T. - Homotopy theory, Academic Press, New York, 1959.

[8] Milnor, J. - Whitehead Torsion, Bull. AMS 72, 1966, 358-426.

[9] Munkres, J.R. - Elements of Algebraic Topology, The Benjamin/Cummings Publishing Company, Inc., California, 1984.

[10] Rotman, J.J. - An Introduction to the Theory of Groups, Graduate Texts in Mathematics No. 148, Springer-Verlag, 1994. 
[11] Schubert, H. - Topology, Allan and Bacon Inc., Boston, 1968.

[12] Siebenmann, L. C. - Infinite simple homotopy types, Indag. Math., $32, \mathrm{~N}^{\circ} 5,1970$.

[13] Spanier, E. H. - Algebraic Topology, McGraw-Hill Book Co., New York, 1966.

[14] Stallings, J. - Whitehead torsion of free products, Annals of Math. 82, (1965) 354-363.

[15] Whitehead, G.W. - Elements of Homotopy Theory, Graduate Texts in Mathematics No. 61, Springer-Verlag, 1978.

[16] Whitehead, G.W. - Homotopy Theory, M.I.T. Press, Cambridge, 1966. 